\title{
Integrated Metabolic and Gene Expression Profiling Reveals New Therapeutic Modalities for Rapidly Proliferating Breast Cancers
}

Chengheng Liao

University of Texas Southwestern Medical Center

Cherise Ryan Glodowski

University of North Carolina School of Medicine

Cheng Fan

University of North Carolina School of Medicine

Juan Liu

Duke University

Kevin Raynard Mott

University of North Carolina School of Medicine

Akash Kaushik

University of Texas Southwestern Medical Center

Hieu Vu

University of Texas Southwestern Medical Center

Jason Locasale

Duke University https://orcid.org/0000-0002-7766-3502

Samuel K McBrayer

University of Texas Southwestern Medical Center

Ralph DeBerardinis

The University of Texas Southwestern Medical Center https://orcid.org/0000-0002-2705-7432

Charles Perou ( $\square$ chuck_perou@med.unc.edu )

University of North Carolina School of Medicine

Qing Zhang ( $\nabla$ qing.zhang@utsouthwestern.edu )

University of Texas Southwestern Medical Center https://orcid.org/0000-0002-6595-8995

\section{Research Article}

Keywords: Metabolic Dysregulation, Patient Prognosis, Genomic Biomarker

Posted Date: August 6th, 2021

DOI: https://doi.org/10.21203/rs.3.rs-117384/v2 
License: (c) (i) This work is licensed under a Creative Commons Attribution 4.0 International License. Read Full License 


\section{Integrated Metabolic and Gene Expression Profiling Reveals New Therapeutic Modalities for Rapidly Proliferating Breast Cancers}

Chengheng Liao ${ }^{1,7}$, Cherise Ryan Glodowski2,7, Cheng Fan³, Juan Liü, Kevin R. Mott², Akash Kaushik ${ }^{5}$, Hieu Vư ${ }^{5}$, Jason W. Locasale ${ }^{4}$, Samuel K. McBrayer ${ }^{5}$, Ralph J.

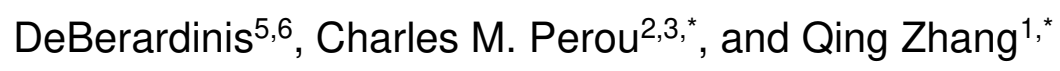

${ }^{1}$ Department of Pathology, University of Texas Southwestern Medical Center, Dallas, TX 75390, USA

${ }^{2}$ Department of Genetics, University of North Carolina School of Medicine, Chapel Hill, NC 27599, USA

${ }^{3}$ Lineberger Comprehensive Cancer Center, University of North Carolina School of Medicine, Chapel Hill, NC 27599, USA

${ }^{4}$ Department of Pharmacology and Cancer Biology, Duke University School of Medicine, Durham, NC 27710, USA.

${ }^{5}$ Children's Medical Center Research Institute, University of Texas Southwestern Medical Center, 5323 Harry Hines Boulevard, Dallas, TX 75390, USA.

${ }^{6}$ Howard Hughes Medical Institute, University of Texas Southwestern Medical Center, 5323 Harry Hines Boulevard, Dallas, TX 75390, USA.

7 These authors contributed equally

* Co-corresponding authors (Q.Z or C.P):

Qing Zhang, Ph.D, Email:Qing.Zhang@UTSouthwestern.edu

Charles Perou, Ph.D, Email:cperou@med.unc.edu

Running title: Metabolic profiling in breast cancer. 


\section{Summary}

This study provides a new stratification of breast tumor samples based on integrated metabolic and gene expression profiling, which guides the selection of newly effective therapeutic strategies targeting breast cancer subsets.

\section{Abstract}

Metabolic dysregulation, although a prominent feature in breast cancer, remains undercharacterized in patient tumors. By performing untargeted metabolomics analyses on triple-negative breast cancer (TNBC) and Estrogen Receptor (ER) positive patient breast tumors, as well as TNBC patient-derived xenografts (PDXs), we identified two major metabolic groups independent of breast cancer histological subtypes: a "Nucleotide/Carbohydrate-Enriched" group and a "Lipid/Fatty Acid-Enriched" group. Cell lines grown in vivo more faithfully recapitulate the metabolic profiles of patient tumors. Integrated metabolic and gene expression analyses reveal genes that strongly correlate with metabolic dysregulation and predict patient prognosis. As a proof-of-principle, targeting Nucleotide/Carbohydrate-Enriched TNBC cell line or PDX xenografts with a pyrimidine biosynthesis inhibitor, and/or a glutaminase inhibitor, led to therapeutic efficacy. In addition, the pyrimidine biosynthesis inhibitor presents better therapeutic outcomes than chemotherapy agents in multiple murine TNBC models. Our study provides a new stratification of breast tumor samples based on integrated metabolic and gene expression profiling, which guides the selection of newly effective therapeutic strategies targeting rapidly proliferating breast cancer subsets. In addition, we develop a public, interactive data visualization portal (http://brcametab.org) based on the data generated from this study. 


\section{Introduction}

Breast cancer is the most commonly diagnosed malignancy and $2^{\text {nd }}$ most frequent cause of cancer-associated death for women worldwide (Sung et al., 2021). Triple-negative breast cancer (TNBC), which accounts 15-20\% all breast cancers (Anders and Carey, 2009), is a clinically defined breast cancer subtype lacking estrogen and progesterone hormone receptor (ER and PR) expression and human epidermal growth factor receptor 2 (HER2) gene amplification (Anders and Carey, 2009). TNBC is featured by clinically aggressive and heterogeneous, highly metastatic, and shorter survival times compared to other subtypes such as ER ${ }^{+}$and HER2 ${ }^{+}$breast cancers (Anders and Carey, 2009). The absence of a valuable therapeutic target in TNBC leaves the patients with limited treatment options, which is mostly focused upon canonical chemotherapy. Therefore, there is an urgent need to identify novel targets in TNBC.

Tumor cells often undergo metabolic alterations to fulfill the high demands of uncontrolled cell proliferation and tumorigenesis, which is a hallmark of cancer (Hanahan and Weinberg, 2011; Ward and Thompson, 2012). Therefore, tumor cell metabolism can be considered cancer's Achilles' heel and is a proven target of successful therapies (Kroemer and Pouyssegur, 2008; Pavlova and Thompson, 2016; Vander Heiden, 2011). Cancer metabolic alterations include reprogramming of glycolysis, glutaminyl's, oxidative phosphorylation (OXPHOS), fatty acid metabolism, etc., which provide essential energy, biosynthesis intermediates, division and redox homeostasis for tumor growth (Pavlova and Thompson, 2016). Therefore, a better understanding of breast tumor cell metabolism can potentially lead to novel therapeutic strategies through targeting specific metabolic pathways dysregulated in tumors while sparing the normal cells. Recently, several studies 
suggest that there is a unique metabolic dependence in subsets of TNBCs, including TNBCs in patients undergoing chemotherapy or displaying PI3K/Akt hyper-activation (Brown et al., 2017a; Lien et al., 2016; Mathur et al., 2017). However, it remains unclear how we can potentially target the specific metabolic vulnerabilities in TNBC compared to other subtypes of breast cancer. In addition, given the well-known heterogeneity of TNBC, it remains unclear whether we can stratify TNBC patients according to their metabolic abnormalities and design rational metabolic therapeutic modalities against subsets of TNBC.

Our study comprehensively investigated the metabolic phenotypes, as well as gene expression phenotypes, of breast tumor patient samples, TNBC patient-derived xenografts (PDXs), and cell lines grown in vitro and in vivo from representative breast cancer subtypes including TNBC and ER ${ }^{+}$breast cancers. In this array of systematic analyses, we identified the specific metabolites that are enriched in $\mathrm{ER}^{+}$tumors and TNBCs. We also examined the correlation between metabolites and gene expression features. Lastly, we performed functional validation experiments by targeting the dysregulated metabolic pathways pharmacologically in multiple TNBC models.

\section{Results}

\section{Metabolic Profiling of Patient Breast Cancer Tumors and TNBC PDXs}

To systemically examine the metabolic profiles in TNBC and $\mathrm{ER}^{+}$breast cancer, we obtained 15 TNBC and $9 \mathrm{ER}^{+}$flash frozen breast tumor samples; we also collected 7 flash frozen tumor samples from 2 TNBC PDX models (WHIM2 and WHIM30) grown in NOD Scid Gamma-deficient (NSG) mice (Li et al., 2013) (see Table S1 for details). We first extracted polar metabolites from a portion of these tumors followed by untargeted 
metabolomics analysis by liquid chromatography, high-resolution mass spectrometry (LC-MS) (Fig. 1A). A total of 301 metabolites were detected in all 31 tumor samples with 263 well annotated by the Kyoto Encyclopedia of Genes and Genomes (KEGG) database (Kanehisa and Goto, 2000) or the Human Metabolome Database (HMDB) (Wishart et al., 2018) (Fig. 1B). Inquiring whether the global metabolic profiling could reveal differences in distinct breast cancer subsets, we performed an unsupervised hierarchical clustering using the 263 annotated metabolites data from the 31 tumor samples (Fig. 1C; Table S2). The cluster divided these patients into two distinctive metabolic clusters (Fig. 1C). Intriguingly, 7/15 TNBC tumors clustered with all of $\mathrm{ER}^{+}$breast tumors (Cluster 1) while all TNBC PDXs tumors grouped with 8/15 TNBC tumor samples (Cluster 2). The clustering pattern for these breast tumor samples suggests metabolic heterogeneity of the TNBC tumors and a more homogenous feature of $\mathrm{ER}^{+}$tumors in terms of metabolite abundance. We also performed mRNA sequencing (mRNAseq) on all 31 tumor samples and applied PAM50 subtyping based on gene expression to further interrogate the tumor molecular features (Parker et al., 2009). As expected, all TNBC PDXs and most TNBC tumors were categorized as basal-like breast cancer (9 out of 15), and most basal-like TNBCs (i.e., 6/9), together with the 7 basal-like PDXs, were present in the Cluster 2 (Fig. 1C), while all of the $\mathrm{ER}^{+}$and some of TNBC from Cluster 1 were luminal or normal-like subtype by the PAM50 analysis (Fig. 1C). We then performed a supervised statistical analysis (SAM (Significance Analysis of Microarrays), see Methods for details) to the metabolite data to identify the significantly altered metabolites between the two metabolic clusters (Fig. S1A). Among the 263 annotated metabolites, 93 were significantly higher in 
Cluster 1 (35.3\%; q<0.05, SAM), and 90 were significantly higher in Cluster 2 (34.2\%; q<0.05, SAM) (Fig. 1D; Fig. S1, B and C).

To systematically investigate the metabolic alterations in the breast cancer patient tumors and PDXs stratified by the metabolic clusters, we performed a KEGG pathway-based differential abundance (DA) analysis (Hakimi et al., 2016) using the metabolites significantly varied between Cluster 1 and 2 tumors. The DA scores were calculated based on the average, gross changes for all metabolites in a pathway. Given the small number detected by LC-MS but important role of some metabolites in some pathway, pathways with at least 2 detectable metabolites were included for the DA analysis and this captured 49 metabolic pathways as being differentially abundant with a broad metabolic pathway category (Fig. 1E). Interestingly, most of the pathways elevated in the Cluster 1 tumors were lipid/fatty acid metabolism and some amino acid metabolism pathways such as glycine, serine and threonine metabolism, whereas pathways elevated in the Cluster 2 tumors were significantly enriched with pyrimidine and purine metabolism, energy production and carbohydrate metabolism (Fig. 1E). We also conducted integrated pathway enrichment and topology analysis (PA) by MetaboAnalyst (Chong et al., 2019), which considered the metabolite impact in each pathway and found some common pathways from both DA and PA analyses in both clusters (Fig. S2, A and B). Additionally, these pathway-based analyses were further illustrated by the differential abundance of individual key metabolites for each pathway category in these two clusters (Fig. 1C, F-I; Fig. S2, C-F). Given the overall enrichment of lipid/fatty acid metabolites in Cluster 1 and nucleotide/carbohydrate metabolites in Cluster 2 (Fig. 1C, F-H), we annotated these clusters "Lipid/Fatty Acid-Enriched" for Cluster 1 and "Nucleotide/Carbohydrate-Enriched" 
for Cluster 2 to highlight the metabolic features in these groups of patient tumors and PDXs. We also obtained 9 flash frozen normal breast tissues and performed metabolic profiling (Fig. S3A) and found that these normal breast tissue samples clustered together with $\mathrm{ER}^{+}$breast tumor samples (Cluster 1) and did not change the overall distribution of the tumor sample clustering pattern or pathway enrichment in two clusters (Fig. S3, B-D). Taken together, our metabolic profiling in breast cancer patient tumors clearly classifies a subset of tumors in the Nucleotide/Carbohydrate-Enriched cluster enriched for basallike/TNBC patient tumors and distinct from the Lipid/Fatty Acid-Enriched cluster that contained the other subtypes of breast cancers (and true normal).

\section{Distinctive Metabolism of Breast Cancer Cell Lines Grown In Vivo Versus In Vitro}

Next, we asked whether the metabolic alteration observed in breast cancer patients was represented in cell lines in a subtype-specific and growth-context manner. To this end, we obtained representative breast cancer cell lines from 4 ER+ (MCF-7, T47D, ZR-75-1 and HCC1428) and 4 TNBC (MDA-MB-231, MDA-MB-468, HCC70 and HCC1806) cell lines. These cell lines were either implanted orthotopically in NSG mice (in vivo growth, $\mathrm{n}=3$ mice/line) as described previously (Zhang et al., 2015; Zhang et al., 2009) or cultured in parallel in petri dishes (in vitro growth, $\mathrm{n}=2$ biological duplicates/line). When cell line xenograft tumors reached a common size $(\sim 0.5 \mathrm{~cm}$ in diameter), tumors were harvested and underwent untargeted metabolomics analysis as described above (Fig. 2A; Table S3). It is known that there are metabolic differences for cell lines grown in vivo versus in vitro (Schimke, 1964), and therefore we first examined the data with principal component analysis (PCA), in which the samples were clearly separated by the in vitro and in vivo growth condition (Fig. 2B). Unsupervised hierarchical clustering further showed distinctive 
metabolic profiles between the cell lines grown in in vitro versus in vivo (Fig. 2C), regardless of subtypes of these cells (Fig. S4, A and B). By comparing the metabolic pathway in these two cell growth conditions, metabolites significantly accumulated in vitro were enriched in several metabolic pathways, including amino acid metabolism (arginine biosynthesis, alanine, aspartate and glutamate metabolism, etc.), TCA cycle, and glycolysis/gluconeogenesis (Fig. 2D). Conversely, metabolic pathways enriched in vivo included nucleotide metabolism and fatty acid metabolism (biosynthesis of unsaturated fatty acid and glycerolipid metabolism) (Fig. 2E). Further examination of representative metabolites revealed that glucose, pyruvate, 2-oxoglutarate, succinate, glutamine, and other amino acids were strongly enriched in cells grown in vitro (Fig. 2F) versus many pyrimidine and purine nucleotides which were significantly higher for cells grown in vivo (Fig. 2G). This is expected given that most of these components (such as glucose, glutamine, pyruvate and amino acids) are abundant in the typical DMEM/RPMI cell growth media and readily utilized by cells in the 2-D in vitro growth condition. We also performed analysis to compare in vivo versus in vitro within each breast cancer cell subtype and found very similar pathway enrichment results (Fig. S4, C-F). These findings suggested that in vitro growth dramatically changed the metabolism compared to the in vivo growth in these breast cancer cells, irrespective of the subtype.

\section{Metabolic Profiling of Cell Line Xenograft Grown In Vivo Reveals Similar Metabolite Enrichment as in Patients}

Next, we combined all the metabolomics data from the patient, PDX, and in vitro and in vivo cell lines to examine the metabolic profile. Overall, global unsupervised clustering of all samples suggests that the metabolism in cell lines grown in vivo and PDX tumors is 
more representative of breast tumors from patients (Fig. 3A). Therefore, we focused our analyses on the data of cell lines grown in vivo. We performed supervised comparison between the $\mathrm{ER}^{+}$and TNBC cell lines that revealed 43 and 45 differential abundant metabolites enriched in ER ${ }^{+}$and TNBC cells, respectively (Fig. 3B). PCA and hierarchical clustering analyses by these metabolites suggested two distinct groups between the $\mathrm{ER}^{+}$ and TNBC in vivo cells (Fig. 3, C and D). Pathway analysis for each cell line subtype did not fully recapitulate the metabolic pathway enrichment results from patient tumors (Fig. S5, $A$ and $B$ ), which is possibly due to the relatively fewer metabolites significantly altered between the $\mathrm{ER}^{+}$and TNBC cells, and/or an overall highly proliferative character for these cancer cell lines grown in mice. However, the abundance of some metabolites revealed high similarity between the $\mathrm{ER}^{+}$and TNBC subtypes, as we observed in the metabolic clusters from patient tumors (Fig. 3, E and F; Fig. S5, C-E). These results suggest that pathways such as glucose, glutamine, and lipid/fatty acid metabolism were faithfully altered between the $\mathrm{ER}^{+}$and TNBC subtypes of breast cancer patients and cell lines grown in vivo. Therefore, the cell line in vivo model partially mimics the metabolism seen in actual tumor specimens.

\section{Integrated Analysis of Patient Metabolomics and Transcriptomics Data Reveals Valuable Metabolite and Gene Signatures}

Unique gene expression patterns, including PAM50 subtype and proliferation signatures, show prognostic value for breast cancer patients (Nielsen et al., 2010; Parker et al., 2009). To rigorously evaluate possible relationships between metabolite and gene expression profiles (Fig. 4A), we first calculated median values of the Lipid/Fatty Acid-Enriched metabolite signature from Cluster $1 \quad(n=93$ metabolites, Fig. S1B $)$ and 
Nucleotide/Carbohydrate-Enriched metabolite signature from Cluster 2 ( $\mathrm{n}=90$ metabolites, Fig. S1C) for each of the patient samples, and then compared these values according to their PAM50 subtype. For our data set of 31 tumors and 9 normal breast tissue samples, the Lipid/Fatty Acid-Enriched feature was higher in Luminal A and B samples compared with the basal-like samples, and was especially high in normal-like and true normal breast samples (Fig. 4B). Conversely, the Nucleotide/Carbohydrate-Enriched feature displayed significantly higher values in basal-like subtype tumors (Fig. 4B). Noted that there are 4 TNBC tumors in Cluster 1 with normal-like PAM50 subtype (Fig. 1C) whereas with a Lipid/Fatty Acid-Enriched metabolite profile, further suggesting the potential of using these metabolite signatures to stratify TNBC patients and predicting subtype heterogeneity. Given the known association between PAM50 subtypes and proliferation rates (Nielsen et al., 2010), we next split these samples into tertiles (high, medium, and low) based on the median values of the two metabolite signatures, then plotted gene expression-based proliferation signature values (Fig. 4C). This analysis showed that a high Nucleotide/Carbohydrate-Enriched metabolite profile was significantly associated with high proliferation signature values, while the Lipid/Fatty Acid-Enriched metabolite profile correlated with low proliferation potential. To validate the metabolomic and genomic PAM50 profile relationships, we used genomic and metabolomics data from 32 human breast tumor and 6 normal breast tissue samples from a previous study by Brauer et al. (Brauer et al., 2013) as a validation data set. We used the previously published PAM50 subtype classifications (Brauer et al., 2013) and determined proliferation scores as well as metabolite scores with two clusters as described in the methods. Of the metabolite signatures from our study, 34 out of the 90 metabolites for the 
Nucleotide/Carbohydrate-Enriched metabolite signature and 22 out of 93 metabolites for the Lipid/Fatty Acid-Enriched metabolite signature were present in the validation data set. Using all 32 tumor and 6 normal samples $(n=38)$, the Nucleotide/Carbohydrate-Enriched metabolite signature showed statistically significant differences by expression subtype $(p<0.01)$ and was high in the basal-like tumors in this validation set, which is consistent with the results from our data (Fig. S6A). However, the Lipid/Fatty Acid-Enriched signature did not show statistically significant differences in PAM50 subtypes (Fig. S6A), possibly reflecting the small numbers matched metabolites in this validation set (22/93). We further split the 32 tumor samples into tertiles (high, medium, and low) based on the median values of the Nucleotide/Carbohydrate-Enriched signature and proliferation score, which again showed higher values in highly proliferating tumors (Fig. S6B), thus validating the predictive value of these signatures on a second specimen test set.

To identify genes that correlate with the metabolite signatures, we performed a supervised gene expression analysis based on the metabolic clustering (Fig. 4, A and D). Supervised analysis of gene expression by SAM based on the two metabolic clusters each gave rise to distinctive gene expression profiles and groups of differentially expressed (DE) genes ( $q<0.05$, SAM) (Fig. 4D; Fig. S6C). We herein define these genes as "Lipid/Fatty Acid-Enriched" or "Nucleotide/Carbohydrate-Enriched" associated gene signatures. For the Nucleotide/Carbohydrate-Enriched genes from Cluster 2, this analysis gave 2908 DE genes; when examing the top 200 DE genes, these genes were homogenously and highly expressed in Cluster 2 versus Cluster 1 tumors (Fig. S6D). Gene ontology (GO) analysis using these top 200 DE genes, suggested they correlated strongly with biological processes including cell division, DNA replication, cell cycle, and 
cell proliferation (Fig. 4E). Importantly, when analyzed using the 1100 patients in the TCGA Breast Cancer data set (Cancer Genome Atlas, 2012), this top 200 gene mRNA profile correlated with multiple previously defined proliferation profiles, including the aforementioned PAM50 proliferation signature used in Fig. 4C and Fig. S6B (Pearson correlation 0.93). Next, we performed a similar analysis using the Lipid/Fatty AcidEnriched metabolite profile and identified 6224 DE genes as being associated with this feature $(q<0.05$, SAM). Examining the Lipid/Fatty Acid-Enriched metabolite profile associated top 200 genes (Fig. S6E) by GO analysis revealed that they were involved in angiogenesis, cellular response to hormone stimulus, and response to progesterone (Fig. 4F). These represent some of the canonical pathways generally enriched in the luminal breast cancers (Osborne and Schiff, 2011).

To determine whether these 200 gene signatures predict patient prognosis in breast cancer, we extracted gene expression data from the METABRIC (Curtis et al., 2012) ( $n=1992)$, Harrell 2011 (Harrell et al., 2012) ( $n=855)$, and SCAN-B data sets (Brueffer et al., 2018) $(n=2969)$, then calculated the median expression of the Nucleotide/Carbohydrate-Enriched 200 DE genes followed by Kaplan-Meier (K-M) survival analysis according to tertiles of expression (Fig. 4G). High expression of these 200 genes strongly correlated with worse prognosis, which is expected given their high accordance to known proliferation signatures and the strong, known prognostic value of proliferation features in breast cancer (Gatza et al., 2014; Nielsen et al., 2010). Interestingly, by performing survival analysis in the aforementioned patient datasets when using the Lipid/Fatty Acid-Enriched 200 DE genes, we found that high expression of these genes predicted better prognosis (Fig. 4H). 


\section{Pyrimidine Metabolism and Glutaminolysis Pathways are Elevated in TNBC}

We further probed the relationships between gene expression and metabolite levels using the identified DE genes within each metabolic clusters (Fig. 4D) and performed metabolic focused gene set-based pathway analysis by the DAVID bioinformatics tools (Huang et al., 2009). Interestingly, although there were more DE genes identified in Cluster 1 than Cluster 2 (2908 versus 6224, Fig. 4D), we found that more metabolic pathways were significantly enriched in the samples from Cluster 2 than Cluster 1 (Fig. 5A). Notably, pyrimidine and purine metabolism, carbon metabolism, and the energy production related pathways were highly enriched in Cluster 2 while many lipid/fatty acid metabolism related pathways were enriched in Cluster 1, suggesting the metabolic gene expression is associated with the metabolite abundance in the patient samples. This finding was further demonstrated by the joint-pathway analysis function from MetaboAnalyst using the significantly accumulated metabolites and upregulated DE genes from each cluster (Fig. S7, A and B). Among these analyses, we found that the pyrimidine metabolism was the top pathway (Fig. 5A; Fig. S7B), and its related metabolic genes were upregulated in Cluster 2 TNBC and basal-like subtype tumors (Fig. 5, B-D; Fig. S7B). It is also worth noting that pyrimidine biosynthesis was previously reported to be upregulated in response to chemotherapy exposure in TNBC (Brown et al., 2017a). We also noticed that in all cases of metabolic profiling analyses, TNBC patient tumors in Cluster 2, PDXs, and TNBC cell lines grown in vivo had marked high levels of glutamate and low levels of glutamine (Fig. 1 and 3E). Some of the key glutamine metabolism and transport enzymes gene expression such as the glutaminase (GLS) was upregulated in the Cluster 2 TNBC tumors (Fig. 5, C-E), suggesting the TNBC tumors maintains high level of glutamine metabolism 
compared to $\mathrm{ER}^{+}$breast tumors. In addition, PTEN mutant TNBC cells were dependent on glutamine flux through the de novo pyrimidine synthesis pathway, which rendered these cells to be sensitive to DHODH inhibitor treatment (Mathur et al., 2017). Indeed, our steady-state metabolite profiling in patient tumors suggested that Cluster 2 TNBC tumors maintained high levels of glutaminolysis state with high levels of TCA cycle intermediates, glutamate and other downstream metabolites including arginine, proline, reduced glutathione (GSH), and high levels of de novo pyrimidine biosynthesis intermediates, concomitant with low level of glutamine and aspartate in these tumors (Fig. $5 F)$. By examing the relative ratio both in the patient tumors and cell lines grown in vivo, we showed higher levels of glutamate/glutamine (Fig. 5G), carbamoylaspartate/glutamine (Fig. 5H), and carbamoyl-aspartate/aspartate (Fig. 5I) in TNBC versus $\mathrm{ER}^{+}$breast cancer. These results suggest that the glutaminolysis and pyrimidine metabolism pathways are elevated in TNBC.

It is worth noting that glutamine-dependent catabolism feeds multiple biosynthetic pathways including nucleotides, lipids, TCA cycle, and others (Altman et al., 2016), and a recent study showed that glutamine metabolism was the major energy and nucleotide resources for cancer cells (Reinfeld et al., 2021). To confirm that the glutaminolysis and pyrimidine metabolism are directly from the glutamine metabolism in TNBC, we performed in vivo isotope tracing experiment in a basal-like TNBC cell xenograft model. Bolus intravenous injection of ${ }^{13} \mathrm{C}_{5}$-glutamine through tail vein within 45 mins (Leone et al., 2019) led to a rapid glutamine pool labeling in the HCC1806 xenograft tumors (Fig. 5J). Notably, bolus infusion also revealed significant pool labeling of glutamate and aspartate but not other amino acids (Fig. 5J). We also noticed high levels of labelled TCA 
cycle intermediates such as succinate, fumarate, and citrate, but minimal labeling of GSH or GSSG (Fig. 5K). Intriguingly, some of the pyrimidine nucleotides and nucleotide sugars were also labelled in the carbon backbone through ${ }^{13} \mathrm{C}_{5}$-glutamine infusion, which may largely be due to the indirect fueling from labelled aspartate (Fig. $5 \mathrm{~L}$ ). To directly confirm the fueling from glutamine to de novo pyrimidine biosynthesis, we also bolus infused the HCC1806 xenograft with ${ }^{15} \mathrm{~N}_{2}$-glutamine and observed significant labelling of the pyrimidine nucleotides in 45 mins (Fig. 5M). Altogether, these results demonstrate that glutamine catabolism-based pyrimidine metabolism and glutaminolysis pathways are highly required in TNBC.

\section{Combinatorial Targeting of Pyrimidine and Glutamine Metabolism in TNBC Models}

Next, we sought to explore therapeutic opportunities by targeting the pyrimidine and/or glutamine metabolism in TNBC models. To target glutamine metabolism, we used the

drug CB-839 to inhibit the GLS enzyme, and to target pyrimidine metabolism, we used the drug Brequinar to inhibit $\mathrm{DHODH}$, the enzyme that converts dihydroorotate to orotate (Fig. 6A). To understand how glutamine metabolism changes in TNBC cells upon CB-839 treatment, we bolus-infused the $\mathrm{HCC} 1806$ tumors with ${ }^{13} \mathrm{C}_{5}$-glutamine with or without $\mathrm{CB}$ 839 treatment. We found significant decrease of the labeled glutamate (Fig. 6B) and its downstream metabolites related to glutamate metabolism (Fig. 5F), including aspartate, proline, and arginine biosynthesis intermediates (Fig. 6B), as well as TCA cycle intermediates and glutathione (Fig. 6C) by CB-839 treatment. Similarly, we bolus-infused the HCC1806 tumors with ${ }^{15} \mathrm{~N}_{2}$-glutamine with or without Brequinar treatment and found decrease of labeled intermediates from de novo pyrimidine synthesis pathway, including 
orotate or UMP by Brequinar treatment (Fig. 6D). These data suggest on-target effects of these drug treatments in our TNBC model.

To examine the therapeutic efficacy of targeting pyrimidine and glutamine metabolism in vivo, we first separately implanted two different basal-like TNBC cell lines, fast-growing HCC1806 and slower-growing MDA-MB-468, orthotopically into the mammary fat pad in NSG mice (Fig. 6, E and J). Upon tumor growth to approximately $150 \mathrm{~mm}^{3}$ for HCC1806 and $50 \mathrm{~mm}^{3}$ for MDA-MB-568, mice were randomized into control, CB-839, Brequinar, and CB-839 and Brequinar combined treatment groups. CB-839 was administered continuously in a CB-839 formulated chow (1400mg/kg diet dose) and Brequinar was given intraperitoneally (20mg/kg body weight) twice a week (Fig. 6, E and J). We monitored the tumor growth over time and recorded the survival days upon tumors reaching the end point $(2 \mathrm{~cm}$ in diameter $)$.

For the HCC1806 xenografts, we did not observe overt toxicity reflected by relatively stable body weight for these single and combination treatments (Fig. S8A). CB-839 or Brequinar single treatment led to a decrease in HCC1806 tumor growth (Fig. 6F), and improved survival compared to the control group (Fig. 6G). The combination of both drugs further suppressed the tumor growth over time and significantly prolonged the survival compared to the control or any single treatment group (Fig. 6, F and $\mathrm{G}$ ). In order to examine the effect of the combination drug treatment on tumor metabolism in vivo, we treated mice with established HCC1806 orthotopic tumors (tumor volume $\sim 1000 \mathrm{~mm}^{3}$ ) with or without combination treatment for 10 days followed by tumor collection and targeted metabolite analysis by LC-MS. We found that the metabolites closely related to the CB-839 or Brequinar inhibited pathways were significantly changed by the 
combination drug treatment (Fig. 6A), e.g., glutamine was accumulated, whereas its downstream metabolites including glutamate, TCA cycle intermediates, GSH, and GSSG were decreased by the combined drug treatment (Fig. 6H). On the other hand, the immediate upstream substrate metabolite of DHODH enzyme, dihydroorotate was dramatically accumulated concomitant with decreased downstream pyrimidine nucleotides (Fig. 6I). Therefore, not only did these analyses demonstrate a drug on-target effect, but also provide a proof-of-principle for combining the CB-839 with Brequinar in vivo. We repeated the same set of xenograft experiments above in another TNBC/basal like breast cancer cell line MDA-MB-468 and found a very similar pattern (Fig.6, J-N; Fig. S8B, S9, A-F).

To further test the efficacy of these drugs in a more physiologically relevant model, we treated WHIM2, a representative basal-like TNBC PDX model (Ding et al., 2010) (Fig. $60)$. Our results showed that Brequinar showed anti-tumor efficacy, and that the combined treatment caused decreased tumor growth and prolonged survival when compared to control group or CB-839 treatment group (Fig. 6, P and Q). To further address the metabolite change in tumors over time, metabolites in the PDX tumors with acute (24 hour) or middle-term (15 days) single drug treatment were measured, which further demonstrated the on-target efficacy of these drugs reflected by related metabolite change (Fig. 6, R and S).

\section{Comparison of Pyrimidine Metabolism Inhibition with Standard Chemotherapy}

Previous studies targeted the pyrimidine synthesis in the PTEN-mutant or chemotherapy resistant tumors, which showed promising therapeutic outcomes in TNBC (Brown et al., 2017b; Mathur et al., 2017). Given that pyrimidine metabolism inhibition by Brequinar 
showed significant antitumor efficacy in our TNBC tumor models, we further sought to compare the therapeutic efficacy of Brequinar with standard chemotherapy in TNBC in immune competent models. The Trp53-null genetically engineered transplant mouse model, which produces phenotypically and genomically diverse tumors, can be classified into three major subtypes/classes based on gene expression profiles: p53null-Basal, Claudin-low, and p53null-Luminal (Pfefferle et al., 2013). Here, we utilized 5 validated p53-null serial transplant TNBC models, namely 2336R, 9263-3, 2224L, 2225L, and T11, to test the efficacy of Brequinar and traditional chemotherapy. In the chemotherapy (Carbo/Tax) treatment group, tumor bearing mice were intraperitoneally administered Paclitaxel (10mg/kg) and Carboplatin (50 mg/kg) once per week (Fig. 7A). Tumor growth was monitored and tumor volume was measured upon the control group reached tumor burden, and survival studies were conducted with continued drug treatment over time. We found that each p53-null murine tumor model showed different levels of sensitivity to Brequinar or Carbo/Tax treatment (Fig. 7, B-F). For the Brequinar treatment, all models (2336R, 9263-3R, 2224L, 2225L, and T-11) were sensitive with significantly decreased tumor volume and prolonged survival (Fig. 7, B-F). However, with the Carbo/Tax treatment, 3 models (2336R, 2225L, and T-11) were sensitive (Fig. 7, D-F) and 2 models (9263-3R, 2224L) were resistant (Fig. 7, B and C). More importantly, Brequinar treatment showed better therapeutic outcomes than Carbo/Tax in these models and less toxicity (Fig. 7, B-F). These data suggest that pyrimidine metabolism inhibition by Brequinar shows comparable, or even better, antitumor activity compared with a standard chemotherapy doublet in this array of heterogenous murine TNBC models.

\section{Discussion}


Our study provides a comprehensive metabolic profiling and gene expression analysis of breast cancers comparing TNBC and $\mathrm{ER}^{+}$patient tumors, PDXs, and cell lines in vivo. Based on the data generated from this study, we developed a public, interactive data visualization portal (http://brcametab.org). This portal enables users to interactively explore associations between metabolite abundances and gene expression level within different clinical and PAM50 subtypes of breast cancer samples. We found two distinctive groups of patient samples stratified by unsupervised analyses of the untargeted metabolite data. One of these groups, which was comprised of normal breast tissues, $\mathrm{ER}^{+}$and some TNBC tumor samples, was denoted the "Lipid/Fatty Acid-Enriched" metabolite phenotype because of its high enrichment for lipid/fatty acid related metabolism pathways. Of note, the majority of TNBC tumors and all PDXs tested were featured in the second group, denoted "Nucleotide/Carbohydrate-Enriched" because of its high levels of nucleotide, carbohydrate, and energy metabolism pathways. Furthermore, we found very a strong gene expression signature correlated with each metabolite signature, suggesting that these metabolites may be used as a strong predictor for high cell proliferation rates in breast cancer patients. Therapeutically, we tested the efficacy in different models of TNBC xenografts by blocking two metabolic pathways (pyrimidine metabolism and glutamine/glutamate metabolism) that are robustly altered and upregulated in TNBCs. We also provided the rationale for inhibiting these two pathways through combination treatments, showing improved therapeutic outcomes when compared with single drug treatment in vivo.

Previous research has attempted to identify therapeutic vulnerabilities in metabolic pathways in TNBCs. For example, by using cell line models grown in vitro, researchers 
showed that a core set of TCA cycle and fatty acid pathways could be important for TNBC cell line survival (Lanning et al., 2017). The potential caveat is that this study only used the cell line grown in vitro, which, as we show in our current study, displayed distinctly different metabolic profiles when compared to breast tumor patient samples or even these same cell lines grown in vivo (Fig. 3A). Another study using representative mouse breast cancer models, including PyMT, Wnt1, Neu and C3-TAg model, showed that C3-TAg, a mouse TNBC tumor line with gene expression similar to human basal-like subtype tumors (Pfefferle et al., 2013), displayed decreased lipids and $\gamma$-glutamyl amino acids with increased glycogen metabolites (Dai et al., 2018). This study suggests that increased glutathione production or decreased glutathione breakdown may be important in TNBC, which is consistent with the findings in our study (Fig. 1E and Fig. S7B). Another study used $204 \mathrm{ER}^{+}$and $67 \mathrm{ER}^{-}$breast tumors for metabolomics and revealed that 19 metabolites showed different levels between these two clinical subtypes (Budczies et al., 2013). The metabolite changes included increased beta-alanine, 2-hydroxyglutarate (2$H G)$, glutamate, and decreased glutamine in the ER- breast tumors. This latter finding is consistent with our finding that glutamate metabolism is enriched in TNBC breast tumors. Another study using a limited number of TCGA breast tumor samples showed that ERbreast tumors displayed high levels of 2-HG and tryptophan metabolite kynurenine (Tang et al., 2014). Our study also revealed significant increase in 2-HG in TNBC tumors, PDXs, as well as TNBC cells lines grown in vivo (Fig. 3E; and Fig. S2C). It was also reported that Warburg-like metabolism was enriched in breast tumors exemplified by the increase Glut-1 expression (Brauer et al., 2013; Sun et al., 2020). In accordance with this finding, we also found increased levels of lactate in TNBC tumors and PDXs compared to ER ${ }^{+}$ 
breast tumors. Lactate is one of the most important metabolites involved in glycolysis and has been reported to drive cancer progression in different cancer settings (Faubert et al., 2017; Hirschhaeuser et al., 2011; Tasdogan et al., 2020). Its enrichment in the TNBC samples cross-validated our metabolomics results. Besides these, we also see that other metabolites involved in glutamate and pyrimidine metabolism were increased in TNBC tumors, including glutamate, succinate, fumarate, UMP, UDP, CDP, and dUMP, etc. Therefore, it is reasonable to speculate that these metabolites may promote cell proliferation in a similar fashion as lactate. Future investigation will need to be carried out to examine their roles in driving TNBC tumor progression.

Importantly, our study here is one of the very few that have integrated metabolomics and gene expression analysis in breast cancer. It has long been debated whether metabolite dysregulation may play a role in driving breast cancer or whether metabolites may be just the product of dysregulated cell proliferation. Here, we performed integrated analysis for the metabolomics and gene expression for TNBC breast tumors and PDXs, which showed promising implications. First, hierarchical clustering of the patient metabolomics data divided our tumor samples into two distinctive clusters (i.e., one cluster enriched with nucleotides and another in lipids/fatty acids). The small subset of TNBC patient tumors clustered with $\mathrm{ER}^{+}$or normal tissues showed a different metabolic pattern compared with the other TNBC or PDXs tumors, an observation that highlights how the clinically defined TNBC group is diverse on a molecular level, and that analyses strictly using clinically defined groups can mask important heterogeneity in TNBC. Second, the cluster enriched with TNBC and the two PDXs showed distinctive gene expression differences compared to the other cluster enriched with $\mathrm{ER}^{+}$breast tumors, suggesting we can further use a 
gene expression profile to define these metabolically distinct patient subsets. It is important to note that in the top 200 gene signature comparison between metabolitedefined clusters, the gene signature derived from the Nucleotide/Carbohydrate-Enriched cluster correlated highly with many previously defined cell proliferation signatures that contain many of the enzymes for pyrimidine and purine synthesis, thus showing very different and objective 'omics' methods each landing on a common and strong biological phenotype.

Chemotherapy still remains the standard of care for TNBC patients, although new immunotherapy treatments are beginning to have a role in the metastatic setting. Chemotherapy is effective in early stage TNBC, whereas late stage patients and/or patients that develop metastases are more resistant to chemotherapy, leading to a poor survival times (Garrido-Castro et al., 2019). It is imperative to identify new therapeutic vulnerabilities in TNBC that may be able to, either alone or in combination, improve the survival rate for these patients. Although dysregulated metabolism is considered to be important for cancer progression, including in TNBCs, it lacks systemic characterization. In our study, we performed systemic profiling of metabolomics and gene expression for relevant TNBC tumor samples as well as PDXs. We identified that pyrimidine and glutamine/glutamate metabolism are enriched in TNBC tumors, and that directly interfering in these pathways using targeted agents inhibited TNBC models growth. In conclusion, we identify two therapeutic vulnerabilities present within the often-deadly TNBC cells, and a biomarker in the form of both metabolic and genetic signatures, to identify these patients. Future studies will explore the possibilities of using these inhibitors, alone, together, and/or in combination with chemotherapy, to examine whether 
specifically targeting pyrimidine and/or glutamate metabolism can be used as a true biologically targeted therapeutic approach for patients with TNBC.

\section{Materials and Methods}

\section{Patient Samples}

Breast cancer tumors and normal breast tissue samples were obtained from patients from the UNC Tissue Procurement Facility. Primary tumor and normal tissues were obtained, flash frozen, and then used for metabolite profiling and RNA extraction and sequencing. These tumor specimens were cut in half, and half used to make RNA, and half used for metabolite extraction. These patient samples were de-identified and anonymized. An Institutional Review Board (IRB) exemption has been obtained in this study and thus this was considered non-Human Subjects Research.

\section{Cell Culture}

MDA-MB-231 and MCF-7 cells were cultured in Dulbecco's Modified Eagle's Medium (DMEM) (Gibco 11965118) supplemented with 10\% fetal bovine serum (FBS) and $1 \%$ penicillin-streptomycin. T47D, HCC1428, HCC70, HCC1806, ZR-75-1, MDA-MB-468 cells were cultured in 10\% FBS, 1\% P penicillin-streptomycin RPMI 1640 (Gibco 11875093). All cell lines were obtained from ATCC. Cells were used for experiments within 10-20 passages from thawing. All cells were authenticated via short tandem repeat testing. Mycoplasma detection was routinely performed to ensure cells were not infected with mycoplasma by using MycoAlert Detection kit (Lonza, LT07-218). 


\section{Drugs}

Brequinar (HY-108325) and CB-839 (Synonyms: Telaglenastat, HY-12248) were purchased from MedChem Express. Brequinar was dissolved in 10\% DMSO and 90\% sterilized corn oil. CB-839 drug was formulated in the special diet made by Research Diets Inc. which containing $1400 \mathrm{mg} / \mathrm{kg}$ diet dose of CB-839 drug. Paclitaxel and Carboplatin were purchased from Henry Schein Medical.

\section{Metabolite Extraction}

The flash frozen patient tumors, normal breast tissues, PDX tumors, and cell line xenograft tumors were first cut in small pieces on dry ice, then 30 to $100 \mathrm{mg}$ sections were weighed in a new $2 \mathrm{~mL}$ Eppendorf tube. $500 \mu \mathrm{L} 80 \%$ (vol/vol) methanol (pre-cooled in $-80^{\circ} \mathrm{C}$ ) was added to each tissue sample. The tissue chunk was further broken down on ice to form an even suspension by using a rotor-stator homogenizer (TissueRuptor, Qiagen). An additional $500 \mu \mathrm{L}$ pre-cooled $80 \%$ methanol was added in each tube and vortexed rigorously for $1 \mathrm{~min}$. After incubation on ice for an additional $10 \mathrm{~min}$, the tissue extract was centrifuged at $20,000 \mathrm{~g}$ at $4{ }^{\circ} \mathrm{C}$ for $15 \mathrm{~min}$. The supernatant, normalized to the amount weighed of the samples, was transferred into clean Eppendorf tubes. For metabolite extraction from cell lines grown in vitro, cell lines were grown in 6-well plates for 2 days to $90 \%$ confluence. The culture medium was completely removed, cells were rinsed twice with $2 \mathrm{~mL}$ of cold normal saline solution to remove the medium residue completely. Cells were immediately placed on dry ice, followed by the addition of $1 \mathrm{ml} 80 \%$ (vol/vol) methanol (pre-cooled in $-80^{\circ} \mathrm{C}$ ) to each well. After incubation in $-80^{\circ} \mathrm{C}$ for 15 min, cells were scraped into $80 \%$ methanol on dry ice, transferred to Eppendorf tubes, 
and centrifuged at $20,000 \mathrm{~g}$ for $15 \mathrm{~min}$ at $4^{\circ} \mathrm{C}$. The supernatant was normalized to cell number and transfer into Eppendorf tubes. Finally, the tubes were speed vacuum dried at room temperature then store dry pellet in $-80^{\circ} \mathrm{C}$ freezer for further LC-MS analysis.

\section{Metabolomics/Metabolite Analysis}

The dry pellets were reconstituted into $30 \mu \mathrm{L}$ sample solvent (water:methanol:acetonitrile, $2: 1: 1, v / v)$ and $3 \mu \mathrm{L}$ was further analyzed by liquid chromatography-mass spectrometry (LC-MS). Ultimate 3000 UHPLC (Dionex) was coupled to Q Exactive Plus-Mass spectrometer (QE-MS, Thermo Scientific) for metabolite profiling. A hydrophilic interaction chromatography method (HILIC) employing an Xbridge amide column (100 x $2.1 \mathrm{~mm}$ i.d., $3.5 \mu \mathrm{m}$; Waters) was used for polar metabolite separation. Detailed LC method was described previously (Liu et al., 2014) except that mobile phase A was replaced with water containing $5 \mathrm{mM}$ ammonium acetate $(\mathrm{pH}$ 6.8). The QE-MS was equipped with a HESI probe with related parameters set as below: heater temperature, $120^{\circ} \mathrm{C}$; sheath gas, 30 ; auxiliary gas, 10 ; sweep gas, 3; spray voltage, $3.0 \mathrm{kV}$ for the positive mode and $2.5 \mathrm{kV}$ for the negative mode; capillary temperature, $320{ }^{\circ} \mathrm{C}$; S-lens, 55; A scan range $(\mathrm{m} / \mathrm{z})$ of 70 to 900 was used in positive mode from 1.31 to 12.5 minutes. For negative mode, a scan range of 70 to 900 was used from 1.31 to 6.6 minutes and then 100 to 1,000 from 6.61 to 12.5 minutes; resolution: 70000 ; automated gain control (AGC), $3 \times 10^{6}$ ions. Customized mass calibration was performed before data acquisition. LC-MS peak extraction and integration were performed using commercially available software Sieve 2.2 (Thermo Scientific). The integrated peak area was used to represent 
the relative abundance of each metabolite in different samples. The missing values were handled as described in a previous study (Liu et al., 2014).

\section{Metabolomics Data Analysis}

The 40 patient and PDX samples were extracted, normalized by weight and measured by LC-MS at the same time. The metabolomics data was combined in some cases by removing any missing data and keep only the common metabolites that detected in all samples. The annotated metabolites from Kyoto Encyclopedia of Genes and Genomes (KEGG) Compound database (www.genome.jp/kegg) (Kanehisa and Goto, 2000) and human metabolome database (HMDB; www.hmdb.ca) (Wishart et al., 2018) were used for further analysis. The data matrix was then normalized in the following sequential steps: two-based log transformation, row median centering then followed by column standardization. All Hierarchical clustering was performed using the normalized data by the Cluster 3.0 software and visualized by Java TreeView (1.1.6r4). Hierarchical clustering was performed using the following parameters: similarity measure: Euclidean distance; clustering algorithm: Centroid linkage. Principal component analysis (PCA) and KEGG-based pathway enrichment analysis and pathway topology analysis was applied using the MetaboAnalyst 5.0 (https://www.metaboanalyst.ca/) (Chong et al., 2019).

\section{Differential Abundance (DA) Score}

The differential abundance (DA) score calculation was described in a previous study with some modifications (Hakimi et al., 2016). The DA score captures the tendency for a pathway to have increased levels of metabolites, relative to a control group. Briefly, the 
detected metabolites were first assigned to different metabolic pathway categories based on the KEGG pathways by MetaboAnalyst 5.0 software (Chong et al., 2019). Then a statistical analysis method (SAM analysis has been used in this study; see Statistical Analysis section for detail) was applied to determine which metabolites are significantly increased or decreased in abundance in a pathway between different sample groups. Then, after determining the significant increased/decreased metabolites, the DA score was calculated based on the following formular:

DA score $=($ number of metabolites increased - number of metabolites decreased $) /$ number of measured metabolites in a pathway

Thus, the DA score varies from -1 to 1 . A score of 1 indicates that all metabolites in a pathway increased in abundance, while a score of -1 indicates that all metabolites decreased. Consider a limited detection capability by LC-MS but important role of metabolites for some pathways. Pathways with no less than 2 detected metabolites were used for DA score calculation.

\section{RNA Sequencing}

Total RNA was isolated from tumors, normal breast tissues, and PDXs using the RNeasy Plus Mini kit (Qiagen), with additional on-column DNase treatment to eliminate traces of genomic DNA. mRNAseq libraries were made from total RNA using the Illumina TruSeq mRNA sample preparation kit and sequenced on an Illumina HiSeq 2500 using a 2x50bp configuration. Purity-filtered reads were aligned to the human reference GRCh38/hg38 genome using Spliced Transcripts Aligned to a Reference (STAR) version 2.4.2a1. Transcript (GENCODE v22) abundance estimates were generated by Salmon version 
0.6.02 in '-quant' mode, based on the STAR alignments. Raw read counts for all RNAseq samples were normalized to a fixed upper quartile3. RNAseq normalized gene counts were then log2 transformed (zeros were unchanged), and genes were filtered for those expressed in $70 \%$ of samples.

\section{PAM50 Subtyping}

To determine the intrinsic subtypes, we used clinical biomarker statuses and RNAseq gene expression data from 40 samples that included 9 normal breast tissues, $9 \mathrm{ER}^{+}$ breast tumor samples, 15 TNBC (ER-/HER2-/PR-) breast tumor samples, and 7 individual tumors from 2 TNBC patient-derived xenografts (WHIM2 and WHIM30). To obtain the subtype-related biomarkers from our mRNAseq gene expression data, we first used an ER/HER2 subgroup-specific gene normalization method, using the IHC status assigned to each sample. This normalization was done prior to applying the PAM50 predictor to correct differences in the biological composition and any technical bias between the gene expression of our 40 study samples derived from RNAseq and the Agilent Human Microarrays used to create the original PAM50 UNC232 training set (Parker et al., 2009). After labeling samples with their ER/HER2 status, we then extracted the ER/HER2 subgroup-specific percentile centering genes, with the percentile determined from the subgroup of the clinicopathological classifications (See detailed method description in supplemental methods) (Zhao et al., 2015). We then normalized the expression values of the PAM50 genes present in our 40 samples. After gene normalization, we applied the PAM50 predictor (Parker et al., 2009). This calculated the correlation coefficient to the 
PAM50 centroids, and allowed us to assign following intrinsic molecular subtypes to each sample: Basal-like, HER2-Enriched, Luminal A, Luminal B, and Normal-like signatures.

\section{Proliferation Signature and Calculation}

We determined proliferation scores for each of our 40 tumor samples using the Zhao et al (2015) PAM50 subtyping method (Zhao et al., 2015), which outputs a proliferation score for each sample by utilizes a proliferation signature is based on the average expression of 11 key proliferation genes linked to cell cycle progression described by Nielson et al (2010) (Nielsen et al., 2010). This proliferation signature is known to provide robust, prognostic information for disease-free survival (Nielson et al., 2010). To replicate this proliferation score on our Brauer et al (2013) validation test set, the expression values for the 10/11 expressed proliferation score genes (CDC20, KNTC2, MKI67, PTTG1, RRM2, TYMS, UBE2C, BIRC5, CCNB1, CDCA1) were median-averaged and used as the proliferation scores for correlating metabolite signatures with proliferation potential.

\section{In Vivo Isotope Tracing}

Six-week old female NOD SCID Gamma mice (NSG, Jackson lab) were injected with $1 \times 10^{6}$ viable HCC1806 cells orthotopically into the mammary fat pad. Upon tumors establishment ( 1000 $\left.\mathrm{mm}^{3}\right)$, mice were treated with CB-839 for 3 days or Brequinar for 3 doses of injections before the tracing experiment. The in vivo isotope tracing experiment was described previously (Leone et al., 2019). Briefly, ${ }^{13} \mathrm{C}_{5}$ - or ${ }^{15} \mathrm{~N}_{2}$-glutamine (Cambridge Isotope Laboratories) were dissolved in sterilized saline. $200 \mathrm{uL}$ of $0.2 \mathrm{M}{ }^{13} \mathrm{C}_{5}$ - or ${ }^{15} \mathrm{~N}_{2}$ glutamine solution were injected 3 times with 15-minute interval into the tail vein under 
anesthesia. Mice were euthanized via cervical dislocation at 45 min after first injection. Tumor tissue was rapidly harvested and snap frozen in liquid nitrogen and stored at $-80^{\circ} \mathrm{C}$ prior to metabolite extraction.

Treatment in Orthotopic Tumor Xenografts, TNBC PDXs, and Trp53-null Transplant Model

All orthotopic tumor xenograft animal experiments were in compliance with National Institutes of Health guidelines and were approved by the University of Texas Southwestern Medical Center Animal Care and Use Committee, and all TNBC PDX and Trp53-null transplant model experiments were performed in accordance with approved University of North Carolina (UNC) Institutional Animal Care and Use Committee protocols.

Orthotopic Tumor Xenograft

Six-week old female NOD SCID Gamma mice (NSG, Jackson lab) were used for xenograft studies. Approximately $1 \times 10^{6}$ viable HCC1806 or MDA-MB-468 cells were resuspended a mixture of $50 \mu \mathrm{L}$ Matrigel (Corning, 354234) and $50 \mu \mathrm{L}$ FBS-free growth medium and injected orthotopically into the mammary fat pad of each mouse. Treatment was started when HCC1806 tumors reached the volume of approximately $150 \mathrm{~mm}^{3}$ and MDA-MB-468 tumors reached the volume of approximately $50 \mathrm{~mm}^{3}$, considering tumorigenesis ability of each cell line.

TNBC PDX and Trp53-null Transplant Model 
The TNBC PDX model for treatments used in this study was the WHIM2, obtained from the Washington University in St Louis MO. The NSG mice (NOD SCID GAMMA mice) were obtained from the Jackson Laboratory or supplied in-house by the UNC Animal Services Core (ASC). The Trp53-null transplant model has phenotypically and genomically diverse tumors that can be classified into three major subtypes/classes based on gene expression profiles: p53null Basal, Claudin-low and p53null-Luminal (Pfefferle et al., 2013). The five validated BALB/c Trp53-null serial transplant models used in this study were $2336 \mathrm{R}, 9263-3,2224 \mathrm{~L}, 2225 \mathrm{~L}$, and T-11. The Balb/c female mice used were obtained from the Jackson Laboratory.

\section{Tumor Transplantation for TNBC PDX and Trp53-null Transplant Model}

Tumors were digested with the Miltenyi tumor dissociation kit to establish cell aggregate suspensions. Cell aggregates were subsequently washed in Hank's Balanced Salt solution containing $2 \%$ FBS (HF Media) and resuspended in HF media with 50\% Matrigel prior to transplant into cohort mice. Mice were briefly anesthetized with $2 \%$ isoflurane and tumor cells were injected into the inguinal mammary fat pad. Mice were followed 2-3 times weekly with caliper measurement for the establishment of tumors and upon reaching a diameter of $0.5 \mathrm{~cm}$ were randomly assigned into either treatment or control groups.

\section{Treatment with $C B-839$ and Brequinar}

Prior to treatment, the tumor-bearing mouse cohorts for the orthotopic xenograft, TNBC PDX, and Trp53-null murine tumors were divided into four groups by randomization. Tumor-bearing mice in the treatment group were continuously administered a specially 
formulated chow containing $1400 \mathrm{mg} / \mathrm{kg}$ diet dose of CB-839 or an intraperitoneal (I.P.) injection of $20 \mathrm{mg} / \mathrm{kg}$ Brequinar every 3 or 4 days, or a combination of both. Tumor size was measured by caliper and body weight was recorded every 3 or 4 days. Tumor volumes were calculated with the formula: volume $=\left(L \times W^{2}\right) / 2$, where $L$ is the tumor length and $W$ is the tumor width measured in millimeters. For the survival study, mice were sacrificed when tumor reached $2 \mathrm{~cm}$ in any dimension.

\section{Treatment with Brequinar and Carbo/Tax}

Prior to treatment, the tumor-bearing Trp53-null mice were divided into four groups by randomization. Mice in the Carbo/Tax treatment group were administered intraperitoneal injections of Paclitaxel (10 mg/kg dose) and Carboplatin (50 mg/kg dose) once per week. Tumor bearing mice in the Brequinar treatment group were administered an intraperitoneal injection of Brequinar drug $(20 \mathrm{mg} / \mathrm{kg}$ dose) twice per week. All mice were observed for overall condition and weighed bi-weekly. Throughout the treatment period caliper tumor measurements for all mice groups continued at a 2-3 per week frequency until the conclusion of the study when mice reached tumor burden.

\section{Statistical Analysis}

A statistical method specialized for multi-testing, SAM (Significance Analysis of Microarrays) (Tusher et al., 2001) was applied to identify differential abundant metabolites or differentially expressed genes in the patient samples. Specifically, we used SAM to examine the correlation between abundance of each metabolite compounds or mRNA expression of each genes between different sample groups based on the unsupervised 
clustering. For all SAM analyses, distribution-independent ranking tests (based on the Wilcoxon test) and the sample-wise permutation (default by the samr package) were used to ascertain significance (false discovery rate, FDR $<0.05$ ). The SAM analysis gives a list of significantly upregulated (positive log fold change) or downregulated (negative log fold change) features between different sample groups with a q-value $<0.05$ were considered statistically significance.

All other statistical analysis was conducted using Prism 8.0 (GraphPad Software). All graphs depict mean \pm SEM unless otherwise indicated. Statistical significances are denoted as not significant (ns; $P>0.05$ ), ${ }^{*} P<0.05,{ }^{* \star} P<0.01,{ }^{* * *} P<0.001,{ }^{* * *} P<0.0001$. The numbers of experiments are noted in figure legends. To assess the statistical significance of single metabolites between two groups, we used unpaired two-tail student's $t$-test. For animal experiments comparing more than two conditions, differences were tested by a one-way ANOVA followed by Dunnett's or Tukey's multiple comparison tests.

\section{Data Availability Statement}

The FASTQ files from RNAseq data and the star-salmon upper quartile normalized gene expression matrix are being deposited into the dbGAP (dbGaP Study Accession: phs002396.v1.p1) and GEO database. The accession number will be available before publication. The full set of metabolomics data can be accessed from the Tables S2-3, or from corresponding authors (Dr. Qing Zhang, Qing.Zhang@UTSouthwestern.edu or Dr. Charles Perou, cperou@email.unc.edu) upon request. 


\section{Acknowledgments}

We thank all members of the Zhang and Perou laboratories for helpful discussions and suggestions. This work was supported by Cancer Prevention and Research Institute of Texas (Q. Zhang, CPRIT, RR190058) and ACS Research Scholar Award (Q. Zhang, RSG-18-059-01-TBE), NCI Breast SPORE program (C.M. Perou, P50-CA58223), National Cancer Institute (C.M. Perou, R01-CA148761) and BCRF (C.M. Perou).

Financial support: This work was supported by Cancer Prevention and Research Institute of Texas (Q. Zhang, CPRIT, RR190058) and ACS Research Scholar Award (Q. Zhang, RSG-18-059-01-TBE), NCI Breast SPORE program (C.M. Perou, P50CA58223), National Cancer Institute (Q.Z, R01CA256833. C.M. Perou, R01CA148761) and BCRF (C.M. Perou).

\section{Author contributions}

C.M.P., Q.Z., and C.L. participated in the conception and design of the experiments. C.L. performed the metabolite extraction and data interpretation. C.L. and A.K. performed the isotope tracing experiments. J.L. and H. V. performed the metabolite LC/MS measurement and annotation. C.R.G. and C.L. performed the RNA-sequencing and analysis. C.L. and K.R.M. performed the animal study. C.F. performed the data processing, bioinformatic and statistical analysis. R.J.D., J.W.L. and S.K.M. provided critical advice and comments. C.L., Q.Z., C.R.G., and C.M.P. wrote and revised the paper with comments from all authors. 
Competing interests: C.M.P is an equity stock holder and consultant of BioClassifier

LLC; C.M.P is also listed an inventor on patent applications on the Breast PAM50

Subtyping assay.

\section{References}

Altman, B.J., Z.E. Stine, and C.V. Dang. 2016. From Krebs to clinic: glutamine metabolism to cancer therapy. Nat Rev Cancer 16:749.

Anders, C.K., and L.A. Carey. 2009. Biology, metastatic patterns, and treatment of patients with triple-negative breast cancer. Clin Breast Cancer 9 Suppl 2:S73-81.

Brauer, H.A., L. Makowski, K.A. Hoadley, P. Casbas-Hernandez, L.J. Lang, E. Roman-Perez, M. D'Arcy, A.J. Freemerman, C.M. Perou, and M.A. Troester. 2013. Impact of tumor microenvironment and epithelial phenotypes on metabolism in breast cancer. Clin Cancer Res 19:571-585.

Brown, K.K., J.B. Spinelli, J.M. Asara, and A. Toker. 2017a. Adaptive Reprogramming of De Novo Pyrimidine Synthesis Is a Metabolic Vulnerability in Triple-Negative Breast Cancer. Cancer discovery 7:391-399.

Brown, K.K., J.B. Spinelli, J.M. Asara, and A. Toker. 2017b. Adaptive Reprogramming of De Novo Pyrimidine Synthesis Is a Metabolic Vulnerability in Triple-Negative Breast Cancer (vol 7, pg 391, 2017). Cancer Discov 7:782-782.

Brueffer, C., J. Vallon-Christersson, D. Grabau, A. Ehinger, J. Hakkinen, C. Hegardt, J. Malina, Y. Chen, P.O. Bendahl, J. Manjer, M. Malmberg, C. Larsson, N. Loman, L. Ryden, A. Borg, and L.H. Saal. 2018. Clinical Value of RNA Sequencing-Based Classifiers for Prediction of the Five Conventional Breast Cancer Biomarkers: A Report From the Population-Based Multicenter Sweden Cancerome Analysis Network-Breast Initiative. JCO Precis Oncol 2:

Budczies, J., S.F. Brockmoller, B.M. Muller, D.K. Barupal, C. Richter-Ehrenstein, A. KleineTebbe, J.L. Griffin, M. Oresic, M. Dietel, C. Denkert, and O. Fiehn. 2013. Comparative metabolomics of estrogen receptor positive and estrogen receptor negative breast cancer: alterations in glutamine and beta-alanine metabolism. J Proteomics 94:279-288.

Cancer Genome Atlas, N. 2012. Comprehensive molecular portraits of human breast tumours. Nature 490:61-70.

Chong, J., D.S. Wishart, and J. Xia. 2019. Using MetaboAnalyst 4.0 for Comprehensive and Integrative Metabolomics Data Analysis. Curr Protoc Bioinformatics 68:e86.

Curtis, C., S.P. Shah, S.F. Chin, G. Turashvili, O.M. Rueda, M.J. Dunning, D. Speed, A.G. Lynch, S. Samarajiwa, Y. Yuan, S. Graf, G. Ha, G. Haffari, A. Bashashati, R. Russell, S. McKinney, M. Group, A. Langerod, A. Green, E. Provenzano, G. Wishart, S. Pinder, P. Watson, F. Markowetz, L. Murphy, I. Ellis, A. Purushotham, A.L. Borresen-Dale, J.D. Brenton, S. Tavare, C. Caldas, and S. Aparicio. 2012. The genomic and transcriptomic architecture of 2,000 breast tumours reveals novel subgroups. Nature 486:346-352.

Dai, C., J. Arceo, J. Arnold, A. Sreekumar, N.J. Dovichi, J. Li, and L.E. Littlepage. 2018. Metabolomics of oncogene-specific metabolic reprogramming during breast cancer. Cancer Metab 6:5.

Ding, L., M.J. Ellis, S. Li, D.E. Larson, K. Chen, J.W. Wallis, C.C. Harris, M.D. McLellan, R.S. Fulton, L.L. Fulton, R.M. Abbott, J. Hoog, D.J. Dooling, D.C. Koboldt, H. Schmidt, J. Kalicki, Q. Zhang, L. Chen, L. Lin, M.C. Wendl, J.F. McMichael, V.J. Magrini, L. Cook, 
S.D. McGrath, T.L. Vickery, E. Appelbaum, K. Deschryver, S. Davies, T. Guintoli, L. Lin, R. Crowder, Y. Tao, J.E. Snider, S.M. Smith, A.F. Dukes, G.E. Sanderson, C.S. Pohl, K.D. Delehaunty, C.C. Fronick, K.A. Pape, J.S. Reed, J.S. Robinson, J.S. Hodges, W. Schierding, N.D. Dees, D. Shen, D.P. Locke, M.E. Wiechert, J.M. Eldred, J.B. Peck, B.J. Oberkfell, J.T. Lolofie, F. Du, A.E. Hawkins, M.D. O'Laughlin, K.E. Bernard, M. Cunningham, G. Elliott, M.D. Mason, D.M. Thompson, Jr., J.L. Ivanovich, P.J. Goodfellow, C.M. Perou, G.M. Weinstock, R. Aft, M. Watson, T.J. Ley, R.K. Wilson, and E.R. Mardis. 2010. Genome remodelling in a basal-like breast cancer metastasis and xenograft. Nature 464:999-1005.

Faubert, B., K.Y. Li, L. Cai, C.T. Hensley, J. Kim, L.G. Zacharias, C. Yang, Q.N. Do, S. Doucette, D. Burguete, H. Li, G. Huet, Q. Yuan, T. Wigal, Y. Butt, M. Ni, J. Torrealba, D. Oliver, R.E. Lenkinski, C.R. Malloy, J.W. Wachsmann, J.D. Young, K. Kernstine, and R.J. DeBerardinis. 2017. Lactate Metabolism in Human Lung Tumors. Cell 171:358-371 e359.

Garrido-Castro, A.C., N.U. Lin, and K. Polyak. 2019. Insights into Molecular Classifications of Triple-Negative Breast Cancer: Improving Patient Selection for Treatment. Cancer discovery 9:176-198.

Gatza, M.L., G.O. Silva, J.S. Parker, C. Fan, and C.M. Perou. 2014. An integrated genomics approach identifies drivers of proliferation in luminal-subtype human breast cancer. Nat Genet 46:1051-1059.

Hakimi, A.A., E. Reznik, C.H. Lee, C.J. Creighton, A.R. Brannon, A. Luna, B.A. Aksoy, E.M. Liu, R. Shen, W. Lee, Y. Chen, S.M. Stirdivant, P. Russo, Y.B. Chen, S.K. Tickoo, V.E. Reuter, E.H. Cheng, C. Sander, and J.J. Hsieh. 2016. An Integrated Metabolic Atlas of Clear Cell Renal Cell Carcinoma. Cancer Cell 29:104-116.

Hanahan, D., and R.A. Weinberg. 2011. Hallmarks of cancer: the next generation. Cell 144:646674.

Harrell, J.C., A. Prat, J.S. Parker, C. Fan, X. He, L. Carey, C. Anders, M. Ewend, and C.M. Perou. 2012. Genomic analysis identifies unique signatures predictive of brain, lung, and liver relapse. Breast Cancer Res Treat 132:523-535.

Hirschhaeuser, F., U.G. Sattler, and W. Mueller-Klieser. 2011. Lactate: a metabolic key player in cancer. Cancer research 71:6921-6925.

Huang, D.W., B.T. Sherman, and R.A. Lempicki. 2009. Systematic and integrative analysis of large gene lists using DAVID bioinformatics resources. Nat Protoc 4:44-57.

Kanehisa, M., and S. Goto. 2000. KEGG: kyoto encyclopedia of genes and genomes. Nucleic Acids Res 28:27-30.

Kroemer, G., and J. Pouyssegur. 2008. Tumor cell metabolism: cancer's Achilles' heel. Cancer cell 13:472-482.

Lanning, N.J., J.P. Castle, S.J. Singh, A.N. Leon, E.A. Tovar, A. Sanghera, J.P. MacKeigan, F.V. Filipp, and C.R. Graveel. 2017. Metabolic profiling of triple-negative breast cancer cells reveals metabolic vulnerabilities. Cancer Metab 5:6.

Leone, R.D., L. Zhao, J.M. Englert, I.M. Sun, M.H. Oh, I.H. Sun, M.L. Arwood, I.A. Bettencourt, C.H. Patel, J. Wen, A. Tam, R.L. Blosser, E. Prchalova, J. Alt, R. Rais, B.S. Slusher, and J.D. Powell. 2019. Glutamine blockade induces divergent metabolic programs to overcome tumor immune evasion. Science 366:1013-1021.

Li, S., D. Shen, J. Shao, R. Crowder, W. Liu, A. Prat, X. He, S. Liu, J. Hoog, C. Lu, L. Ding, O.L. Griffith, C. Miller, D. Larson, R.S. Fulton, M. Harrison, T. Mooney, J.F. McMichael, J. Luo, Y. Tao, R. Goncalves, C. Schlosberg, J.F. Hiken, L. Saied, C. Sanchez, T. Giuntoli, C. Bumb, C. Cooper, R.T. Kitchens, A. Lin, C. Phommaly, S.R. Davies, J. Zhang, M.S. Kavuri, D. McEachern, Y.Y. Dong, C. Ma, T. Pluard, M. Naughton, R. Bose, R. Suresh, R. McDowell, L. Michel, R. Aft, W. Gillanders, K. DeSchryver, R.K. Wilson, S. Wang, G.B. Mills, A. Gonzalez-Angulo, J.R. Edwards, C. Maher, C.M. Perou, E.R. Mardis, and 
M.J. Ellis. 2013. Endocrine-therapy-resistant ESR1 variants revealed by genomic characterization of breast-cancer-derived xenografts. Cell Rep 4:1116-1130.

Lien, E.C., C.A. Lyssiotis, A. Juvekar, H. Hu, J.M. Asara, L.C. Cantley, and A. Toker. 2016. Glutathione biosynthesis is a metabolic vulnerability in $\mathrm{PI}(3) \mathrm{K} /$ Akt-driven breast cancer. Nature cell biology 18:572-578.

Liu, X., Z. Ser, and J.W. Locasale. 2014. Development and quantitative evaluation of a highresolution metabolomics technology. Anal Chem 86:2175-2184.

Mathur, D., E. Stratikopoulos, S. Ozturk, N. Steinbach, S. Pegno, S. Schoenfeld, R. Yong, V.V. Murty, J.M. Asara, L.C. Cantley, and R. Parsons. 2017. PTEN Regulates Glutamine Flux to Pyrimidine Synthesis and Sensitivity to Dihydroorotate Dehydrogenase Inhibition. Cancer Discov 7:380-390.

Nielsen, T.O., J.S. Parker, S. Leung, D. Voduc, M. Ebbert, T. Vickery, S.R. Davies, J. Snider, I.J. Stijleman, J. Reed, M.C. Cheang, E.R. Mardis, C.M. Perou, P.S. Bernard, and M.J. Ellis. 2010. A comparison of PAM50 intrinsic subtyping with immunohistochemistry and clinical prognostic factors in tamoxifen-treated estrogen receptor-positive breast cancer. Clinical cancer research : an official journal of the American Association for Cancer Research 16:5222-5232.

Osborne, C.K., and R. Schiff. 2011. Mechanisms of endocrine resistance in breast cancer. Annu Rev Med 62:233-247.

Parker, J.S., M. Mullins, M.C. Cheang, S. Leung, D. Voduc, T. Vickery, S. Davies, C. Fauron, X. He, Z. Hu, J.F. Quackenbush, I.J. Stijleman, J. Palazzo, J.S. Marron, A.B. Nobel, E. Mardis, T.O. Nielsen, M.J. Ellis, C.M. Perou, and P.S. Bernard. 2009. Supervised risk predictor of breast cancer based on intrinsic subtypes. Journal of clinical oncology : official journal of the American Society of Clinical Oncology 27:1160-1167.

Pavlova, N.N., and C.B. Thompson. 2016. The Emerging Hallmarks of Cancer Metabolism. Cell metabolism 23:27-47.

Pfefferle, A.D., J.I. Herschkowitz, J. Usary, J.C. Harrell, B.T. Spike, J.R. Adams, M.I. TorresArzayus, M. Brown, S.E. Egan, G.M. Wahl, J.M. Rosen, and C.M. Perou. 2013. Transcriptomic classification of genetically engineered mouse models of breast cancer identifies human subtype counterparts. Genome Biol 14:R125.

Reinfeld, B.I., M.Z. Madden, M.M. Wolf, A. Chytil, J.E. Bader, A.R. Patterson, A. Sugiura, A.S. Cohen, A. Ali, B.T. Do, A. Muir, C.A. Lewis, R.A. Hongo, K.L. Young, R.E. Brown, V.M. Todd, T. Huffstater, A. Abraham, R.T. O'Neil, M.H. Wilson, F. Xin, M.N. Tantawy, W.D. Merryman, R.W. Johnson, C.S. Williams, E.F. Mason, F.M. Mason, K.E. Beckermann, M.G. Vander Heiden, H.C. Manning, J.C. Rathmell, and W.K. Rathmell. 2021. Cellprogrammed nutrient partitioning in the tumour microenvironment. Nature 593:282-288.

Schimke, R.T. 1964. Enzymes of Arginine Metabolism in Mammalian Cell Culture. I. Repression of Argininosuccinate Synthetase and Argininosuccinase. The Journal of biological chemistry 239:136-145.

Sun, X., M. Wang, M. Wang, X. Yu, J. Guo, T. Sun, X. Li, L. Yao, H. Dong, and Y. Xu. 2020. Metabolic Reprogramming in Triple-Negative Breast Cancer. Frontiers in oncology $10: 428$.

Sung, H., J. Ferlay, R.L. Siegel, M. Laversanne, I. Soerjomataram, A. Jemal, and F. Bray. 2021. Global Cancer Statistics 2020: GLOBOCAN Estimates of Incidence and Mortality Worldwide for 36 Cancers in 185 Countries. CA Cancer J Clin 71:209-249.

Tang, X., C.C. Lin, I. Spasojevic, E.S. Iversen, J.T. Chi, and J.R. Marks. 2014. A joint analysis of metabolomics and genetics of breast cancer. Breast cancer research : BCR 16:415.

Tasdogan, A., B. Faubert, V. Ramesh, J.M. Ubellacker, B. Shen, A. Solmonson, M.M. Murphy, Z. Gu, W. Gu, M. Martin, S.Y. Kasitinon, T. Vandergriff, T.P. Mathews, Z. Zhao, D. Schadendorf, R.J. DeBerardinis, and S.J. Morrison. 2020. Metabolic heterogeneity confers differences in melanoma metastatic potential. Nature 577:115-120. 
Tusher, V.G., R. Tibshirani, and G. Chu. 2001. Significance analysis of microarrays applied to the ionizing radiation response. Proceedings of the National Academy of Sciences of the United States of America 98:5116-5121.

Vander Heiden, M.G. 2011. Targeting cancer metabolism: a therapeutic window opens. Nature reviews. Drug discovery 10:671-684.

Ward, P.S., and C.B. Thompson. 2012. Metabolic reprogramming: a cancer hallmark even warburg did not anticipate. Cancer cell 21:297-308.

Wishart, D.S., Y.D. Feunang, A. Marcu, A.C. Guo, K. Liang, R. Vazquez-Fresno, T. Sajed, D. Johnson, C. Li, N. Karu, Z. Sayeeda, E. Lo, N. Assempour, M. Berjanskii, S. Singhal, D. Arndt, Y. Liang, H. Badran, J. Grant, A. Serra-Cayuela, Y. Liu, R. Mandal, V. Neveu, A. Pon, C. Knox, M. Wilson, C. Manach, and A. Scalbert. 2018. HMDB 4.0: the human metabolome database for 2018. Nucleic Acids Res 46:D608-D617.

Zhang, J., C. Wang, X. Chen, M. Takada, C. Fan, X. Zheng, H. Wen, Y. Liu, C. Wang, R.G. Pestell, K.M. Aird, W.G. Kaelin, Jr., X.S. Liu, and Q. Zhang. 2015. EgIN2 associates with the NRF1-PGC1alpha complex and controls mitochondrial function in breast cancer. The EMBO journal 34:2953-2970.

Zhang, Q., J. Gu, L. Li, J. Liu, B. Luo, H.W. Cheung, J.S. Boehm, M. Ni, C. Geisen, D.E. Root, K. Polyak, M. Brown, A.L. Richardson, W.C. Hahn, W.G. Kaelin, Jr., and A. BommiReddy. 2009. Control of cyclin D1 and breast tumorigenesis by the EgIN2 prolyl hydroxylase. Cancer Cell 16:413-424.

Zhao, X., E.A. Rodland, R. Tibshirani, and S. Plevritis. 2015. Molecular subtyping for clinically defined breast cancer subgroups. Breast Cancer Res 17:29. 


\section{Figure legends}

\section{Figure 1. Metabolic profiling in tumors from breast cancer patient and PDXs.}

(A) Schematic of the experimental design for untargeted global metabolomics analysis in $\mathrm{ER}^{+}$, TNBC breast cancer patient tumors and two TNBC patient-derived xenografts (PDXs) tumors. (B) Pie chart shows the number of metabolites identified and annotated in all samples. The annotated metabolites all with a registered ID either from the Kyoto Encyclopedia of Genes and Genomes (KEGG) database or the Human Metabolome Database (HMDB) which can be further used for pathway analysis. (C) Unsupervised hierarchical clustering heatmap examines global metabolic profiles in $31 \mathrm{ER}^{+} / \mathrm{TNBC}$ patient tumor samples and PDX tumors by using the normalized metabolite intensity. Note the cluster divides the samples in two major metabolic clusters (Cluster 1 and Cluster 2). Clinical subtype and PAM50 subtype for each sample are showed. Selected classes of metabolites are highlighted. (D) Pie chart shows the metabolites that are significant increase in the Cluster 1 or Cluster 2 samples based on the SAM analysis (q-value<0.05). (E) KEGG pathway-based differential abundance (DA) analysis using the significant changed metabolites between Cluster 1 and 2. The DA score reflects the average, gross changes for all detected metabolites in a pathway. A score of 1 indicates all measured metabolites in the pathway increase in the Cluster 2 versus Cluster 1 , and score of -1 indicates all measured metabolites in a pathway decrease. Pathways with no less than two measured metabolites were used for DA score calculation based on the detection capability for some important pathways. (F-I) Relative normalized intensity levels of representative metabolites, involved in energy production $(\mathbf{F})$, nucleotides $(\mathbf{G})$, lipids/fatty acids $(\mathbf{H})$, and amino acids $(\mathbf{I})$, that were differentially abundant between metabolic 
clusters from panel (C). Data are represented as mean \pm standard error of the mean (SEM), two-tailed Student's t-test; ${ }^{*} \mathrm{p}<0.05,{ }^{* *} \mathrm{p}<0.01,{ }^{* * *} \mathrm{p}<0.001,{ }^{* * * *} \mathrm{p}<0.0001$.

Figure 2. Distinctive metabolism of cell lines grown in vivo versus in vitro.

(A) Schematic of the experimental design for metabolic profiling of breast cancer cell lines grown in vitro and in vivo condition. Cell lines grown in vivo were conducted by growing and collecting tumors from three individual mice, cell line grown in vitro were performed in parallel in biological duplicates. (B) Principal component analysis (PCA) of individual samples for the normalized metabolomics data in cell line grown in vivo and in vitro. (C) Unsupervised hierarchical clustering heatmap of normalized metabolomics data in cell line grown in vivo and in vitro. (D and E) Histograms of metabolic pathway enrichment using statistical differed metabolites (SAM, q-value $<0.05)$ that high in cells grown in vitro (D) or high in cells grown in vitro $(E)$. All statistically significant $(p<0.05)$ pathways were showed. (F and $\mathbf{G})$ Relative normalized intensity levels of representative metabolites that are significantly increased in cell line grown in vitro $(\mathbf{F})$ or in vivo $(\mathbf{G})$. Data are represented as mean \pm standard error of the mean (SEM), two-tailed Student's $t$-test; ${ }^{*} \mathrm{p}<0.05$, ${ }^{* *} \mathrm{p}<0.01,{ }^{* * *} \mathrm{p}<0.001,{ }^{* * * *} \mathrm{p}<0.0001$.

Figure 3. Cell line grown in vivo reveals similar metabolites enrichment as in patients.

(A) Unsupervised hierarchical clustering heatmap of the normalized metabolomics data by combining all cell line in vitro and in vivo, patient, PDXs samples. (B) Pie chart shows the metabolites that are significant increase in the $\mathrm{ER}^{+}$or TNBC cell derived xenograft 
tumors based on the SAM analysis (q-value $<0.05$ ). (C and D) PCA analysis (C) and unsupervised hierarchical clustering heatmap (D) of the ER ${ }^{+}$and TNBC cell line derived xenograft tumors using the significant changed metabolites. (E and F) Relative normalized intensity levels of representative metabolites that are significantly changed in cell line grown in vitro versus in vivo. Data are represented as mean \pm standard error of the mean (SEM), two-tailed Student's t-test; ${ }^{*} p<0.05,{ }^{* \star} p<0.01,{ }^{* * *} p<0.001,{ }^{* * *} p<0.0001$, not significant (ns).

Figure 4. Integrated analysis of transcriptional and metabolic signatures in patient tumors.

(A) Schematic of the correlation analysis between metabolomics and gene expression data. (B) The PAM50 subtype correlation with the metabolite signatures from Cluster 1 and Cluster 2 patient samples from this study. (C) Correlation of proliferation score with the tertile median level of the two metabolite signatures from Cluster 1 and Cluster 2 patient samples. (D) A supervised clustering heatmap of the differentially expressed (DE) genes that were highly expressed in the Cluster 1 and Cluster 2 samples. DE genes were identified by SAM analysis between the Cluster 1 and Cluster 2 samples with qvalue $<0.05$. ( $\mathbf{E}$ and $\mathbf{F}$ ) Histogram of gene ontology (GO) analysis showing the top 200 genes that are upregulated in Cluster $2(\mathbf{E})$ or Cluster $1(\mathbf{F})$. Top 15 pathways with statistical significance $(p<0.05)$ were showed. $(\mathbf{G}$ and $\mathbf{H})$ Kaplan-Meier plotters generated by the median expression of the top 200 genes from Cluster $2(\mathbf{G})$ or Cluster $1(\mathbf{H})$ in three different databases. 
Figure 5. Pyrimidine metabolism and glutaminolysis are elevated in TNBC.

(A) Metabolic pathway-based gene set enrichment analysis using the DE genes (qvalue $<0.05$, SAM) within each metabolic cluster. All statistically significant $(p<0.05)$ pathways were showed. (B) Heatmap showed relative expression of genes related to pyrimidine metabolism in the patient tumors and PDXs stratified by the metabolic clusters. Genes upregulated in Cluster 2 samples are shown with statistic markers. (C) A schematic shows the key genes for the initial steps of glutaminolysis and de novo pyrimidine biosynthesis. (D) Relative gene expression of the key genes between Cluster 1 and 2 samples. (E) Heatmap shows relative expression of genes related to glutamine metabolism and transport in the patient tumors and PDXs stratified by the metabolic clusters. Genes upregulated in Cluster 2 samples are shown with statistic markers. (F) Schematic of key metabolites denotes metabolite abundance in tumors in Cluster 1 or Cluster 2. Significant abundant metabolites from SAM analysis (q-value $<0.05$ ) were highlighted, with metabolite in red accumulated in cluster 2 or in blue accumulated in cluster 1 . The expected major labeling patterns of some metabolites from ${ }^{13} \mathrm{C}_{5}$ - or ${ }^{15} \mathrm{~N}_{2}$ glutamine isotope tracing are also showed. (G-I) Relative abundance ratio of the downstream and upstream metabolites from glutamate to glutamine (G), carbamoylaspartate to glutamine $(\mathbf{H})$, and carbamoyl-aspartate to aspartate $(\mathbf{I})$, indicating the metabolic flow at the initial steps in the glutaminolysis and pyrimidine metabolism pathways in patient (left) or cell line in vivo (right) samples. (J-L) Contribution of glutamine carbon to amino acids (J), TCA cycle and glutathione (K), and nucleotides (L) in HCC1806 TNBC xenograft tumors $(\mathrm{N}=4)$ infused with ${ }^{13} \mathrm{C}_{5}$-glutamine in 45 mins. (M) 
Contribution of glutamine nitrogen to nucleotides in HCC1806 TNBC xenograft tumors $(\mathrm{N}=6)$ infused with ${ }^{15} \mathrm{~N}_{2}$-glutamine in 45 mins.

Data are represented as mean \pm standard error of the mean (SEM), two-tailed Student's t-test; ${ }^{*} p<0.05,{ }^{* *} p<0.01,{ }^{* * *} p<0.001,{ }^{* * * *} p<0.0001$, ns, not significant.

Figure 6. Combination targeting the pyrimidine and glutamate metabolism in TNBC cell line xenograft and PDX model.

(A) A schematic shows the key enzymes targeted by CB-839 and Brequinar in the glutamine and pyrimidine metabolism pathway and related key metabolites. (B-C) Contribution of glutamine carbon to amino acids (B), the TCA cycle and glutathione (C) in HCC1806 tumors obtained from vehicle- and CB839-treated mice ( $\mathrm{N}=4 / \mathrm{group})$ at 45 min of ${ }^{13} \mathrm{C}_{5}$-glutamine bolus infusion. (D) Contribution of glutamine nitrogen to pyrimidine nucleotides in HCC1806 tumors obtained from 3 doses of vehicle- and Brequinar-treated mice ( $\mathrm{N}=5 /$ group) at 45 min of ${ }^{15} \mathrm{~N}_{2}$-glutamine bolus infusion. The expected major labeling patterns of some metabolites from ${ }^{13} \mathrm{C}_{5}$ - or ${ }^{15} \mathrm{~N}_{2}$-glutamine isotope tracing are showed in Figure 5F. (E-G) Treatment strategy (E), tumor growth measured by volume $(\mathbf{F})$ and survival (G) of the HCC1806 xenograft cohort ( $\mathrm{N}=10 /$ group). ( $\mathrm{H}$ and I) Related abundance of metabolites related to the glutamine metabolism $(\mathbf{H})$ or pyrimidine metabolism (I) between the control and combination treatment group in HCC1806 xenograft tumors. Mice treated with the drugs for 10 days, then the tumors harvested for metabolite analysis. (J-L) Treatment strategy $(\mathbf{J})$, tumor growth measured by volume $(\mathbf{K})$ and harvest tumor weight at 28 day (L) of the MDA-MB-468 xenograft cohort (Control, $N=10 ; C B-839, N=10$; Brequinar, $\mathrm{N}=9$; Combined, $\mathrm{N}=8$ ). ( $\mathbf{M}$ and $\mathbf{N}$ ) Related abundance of key metabolites in 
the glutamine metabolism (M) or pyrimidine metabolism (N) pathways in MDA-MB-468 xenograft tumors in different treatment for 28 days. (O-Q) Treatment strategy $(\mathbf{O})$, survival (P) tumor growth measured by volume $(\mathbf{Q})$ of the WHIM2 PDX xenograft cohort (Control,

$\mathrm{N}=12$; CB-839, $\mathrm{N}=5$; Brequinar, $\mathrm{N}=8$; Combined, $\mathrm{N}=5$ ). ( $\mathbf{R}$ and $\mathbf{S}$ ) Related abundance of key metabolites in the glutamine metabolism $(\mathbf{R})$ or pyrimidine metabolism (S) pathways in the WHIM2 PDX xenograft tumors in different treatment group. Tumors harvested for metabolite analysis at 24 hours and 15 days after the treatment. Data are represented as mean \pm standard error of the mean (SEM), two-tailed Student's t-test; ${ }^{*} p<0.05,{ }^{* *} p<0.01$ ${ }^{* *} p<0.001,{ }^{* * * *} p<0.0001$, not significant $\left({ }^{*} n s\right)$.

Figure 7. Comparison of pyrimidine metabolism inhibition with standard chemotherapy.

(A) Treatment strategy in five p53null TNBC murine tumor line xenografts, mice were treated with standard-of-care chemotherapy (Carboplatin+Paclitaxel) or Brequinar at indicated dose and time. (B-F) Therapeutic outcome in terms of tumor volume change (left) or survival of the cohort (right) in the 2224L (B), 9263-3R (C), 2225L (D), T11 (E), and $2336 \mathrm{R}(\mathbf{F})$ tumor model. Data are represented as mean \pm standard error of the mean (SEM), two-tailed Student's t-test. 


\section{Figure 1}

A
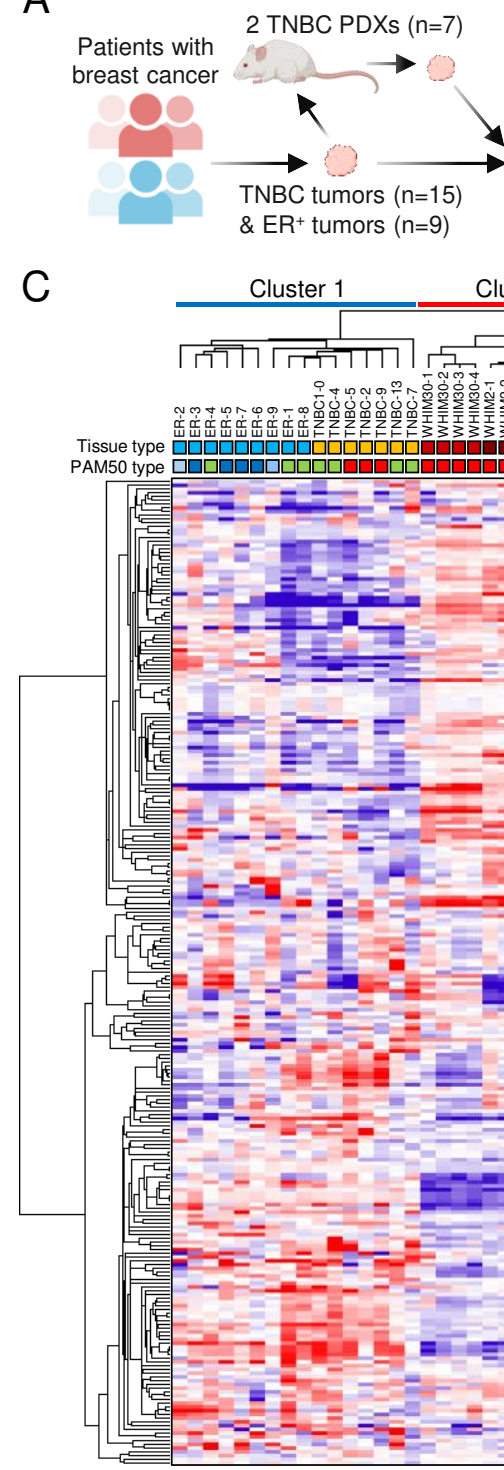

$\mathrm{F}$

$$
\text { H }
$$

B

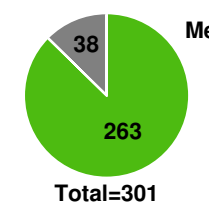

D

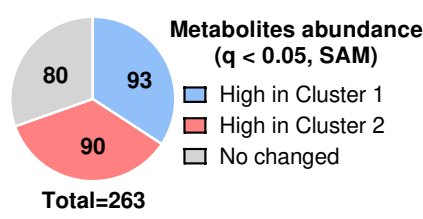

C

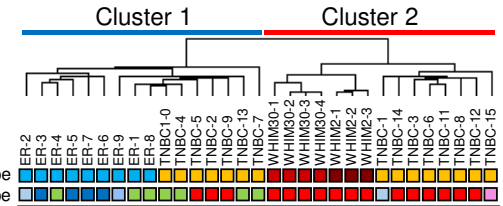
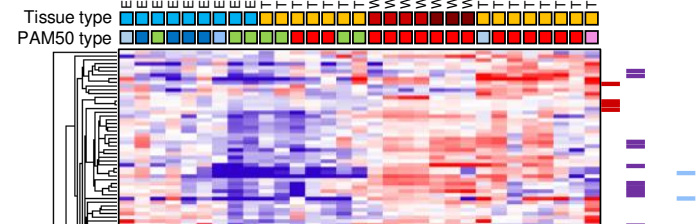

Normalized abundance

T 3.0

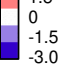

Tissue type

$=\square E R^{+}$

- $\square$ WHIM30-PDX

$\square$ WHIM2-PDX

$\equiv$

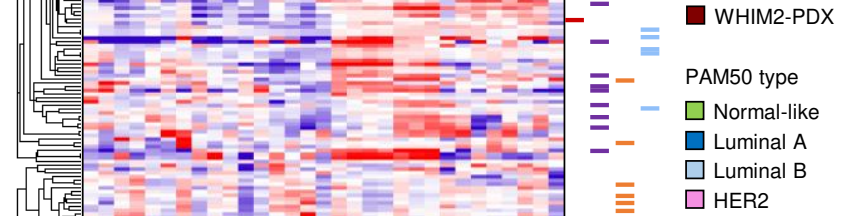

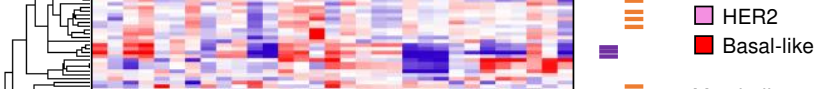
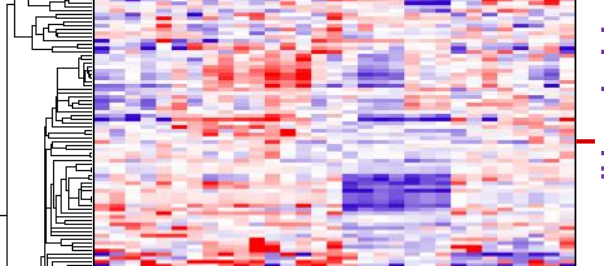

䆣

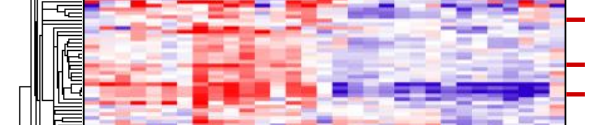

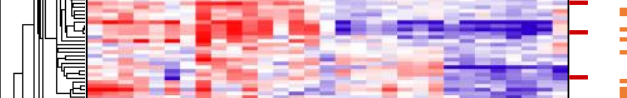

近点 $==$
Metabolite category

$\square$ Energy production relate

$\square$ Nucleotides

$\square$ Lipids/Fatty acids

$=\square$ Amino acids

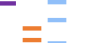

E

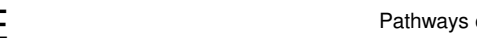

Cluster 1 Cluster 2

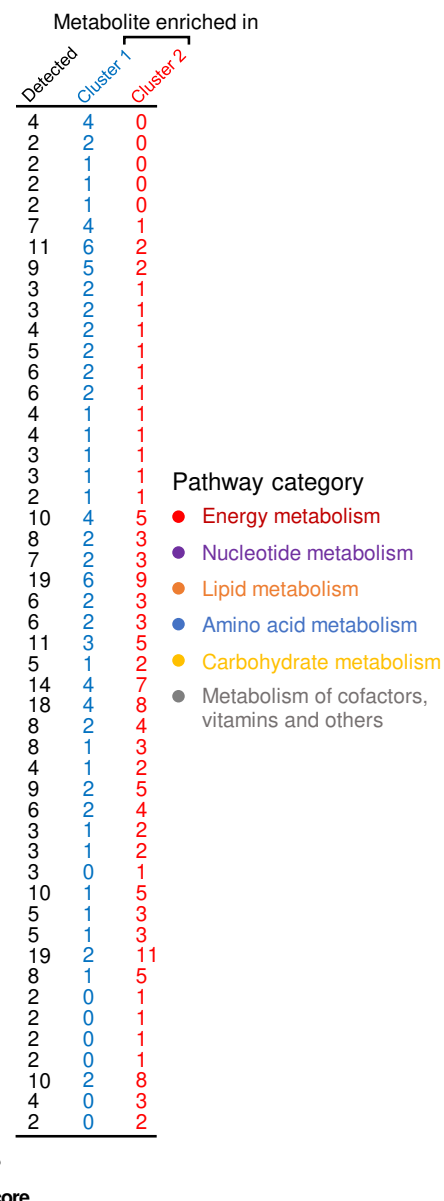

Differential Abundance Score

G

Glycine, serine of unsaturated fatty acid

Glutamine and D-glutamate metabolism

Porphyrin and chlorophyll metabolism

Valine, leucine and isoleucine degradation

aline, leucine and isoleucine biosynthesis Histidine metabolism

Phenylalanine metabolism

Glycerolipid metabolism

Fatty acid degradation
Nitrogen metabolism

Arginine biosynthesis

Citrate cycle (TCA cycle)
Tyrosine metabolism

Purine metabolism

Glycolysis / Gluconeogenesis

Cysteine and methionine metabolism beta-Alanine metabolism

Alanine, aspartate and glutamate metabolism

Aminoacyl-tRNA biosynthesis
Pantothenate and $\mathrm{CoA}$ biosynthesis

Taurine and hypotaurine metabolism

Arginine and proline metabolism

Butanoate metabolism Lysine degradation

Glutathione metabolism

Pyruvate metabolism

Pyrimidine metabolism

Riboflavin metabolism

Phenylalanine, tyrosine and tryptophan biosynthesis Biotin metabolism

Phosphonate and phosphinate metabolism

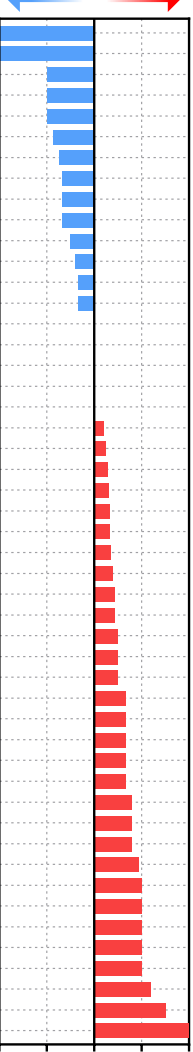

Nucleotides
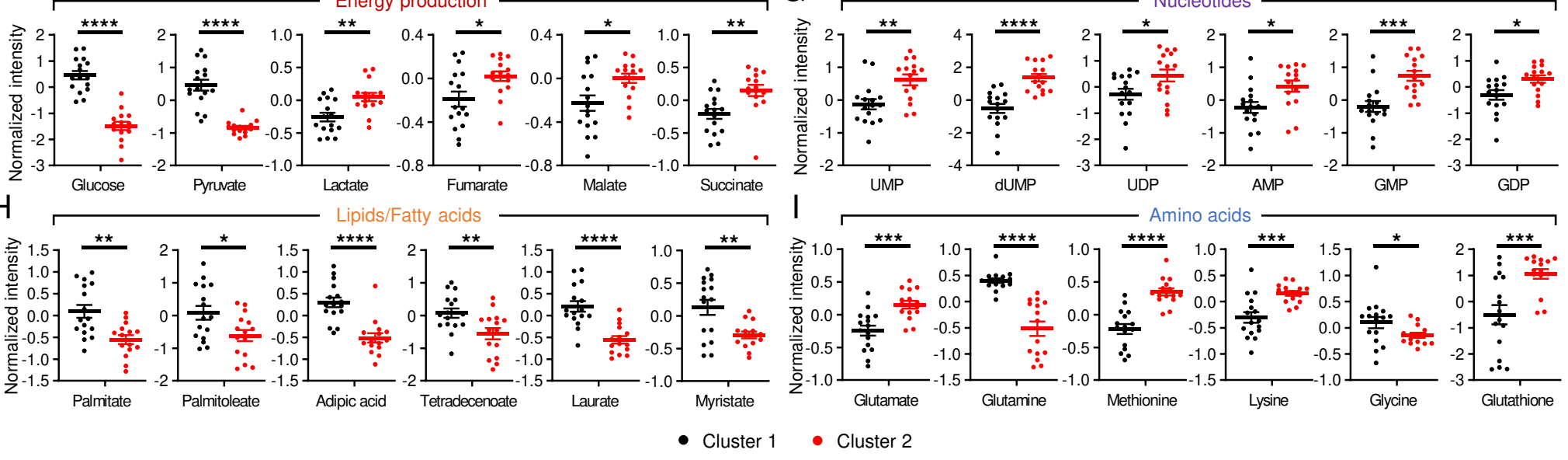
Figure 2

A

B
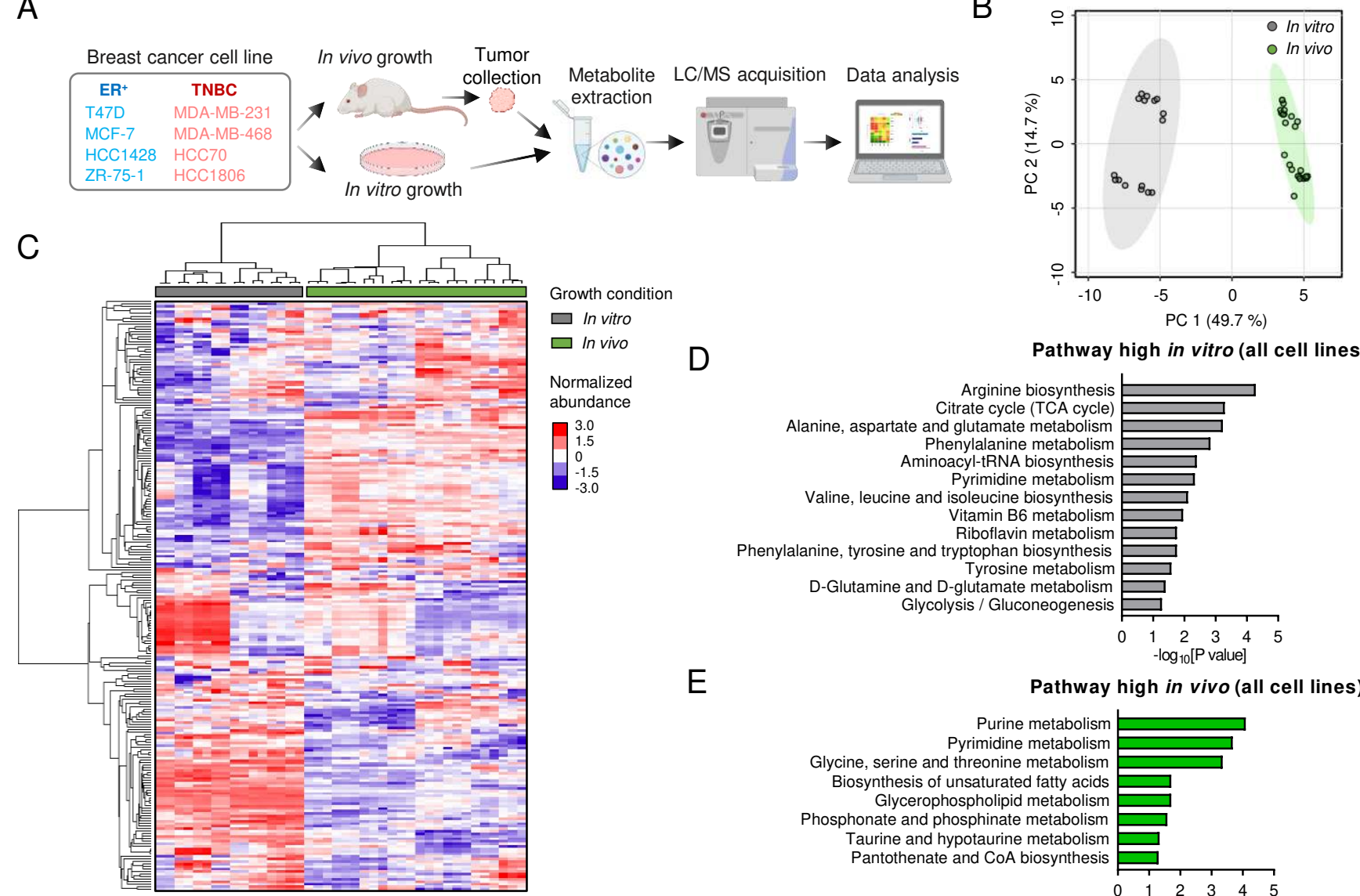

D

Pathway high in vitro (all cell lines)

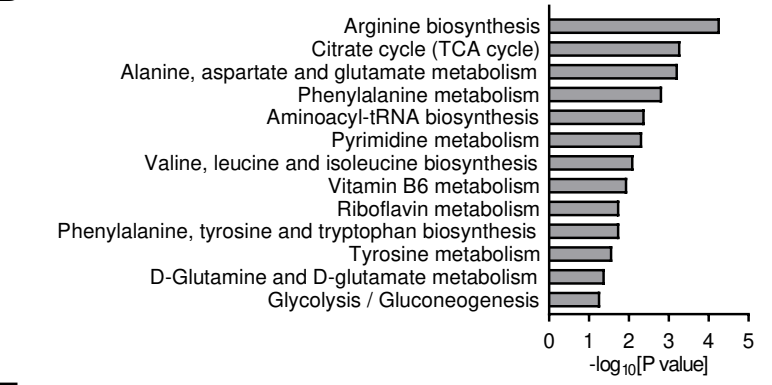

E

Pathway high in vivo (all cell lines)

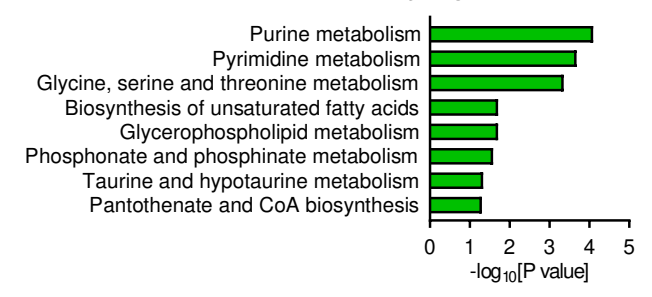

$\mathrm{F}$
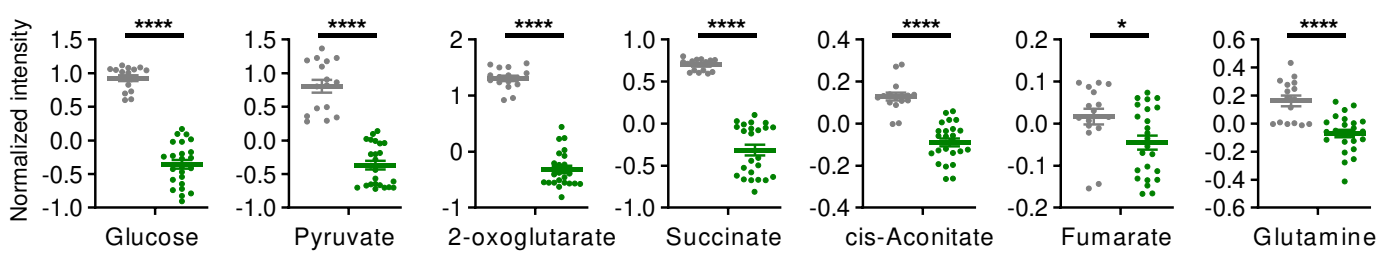

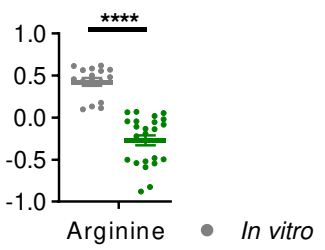

G

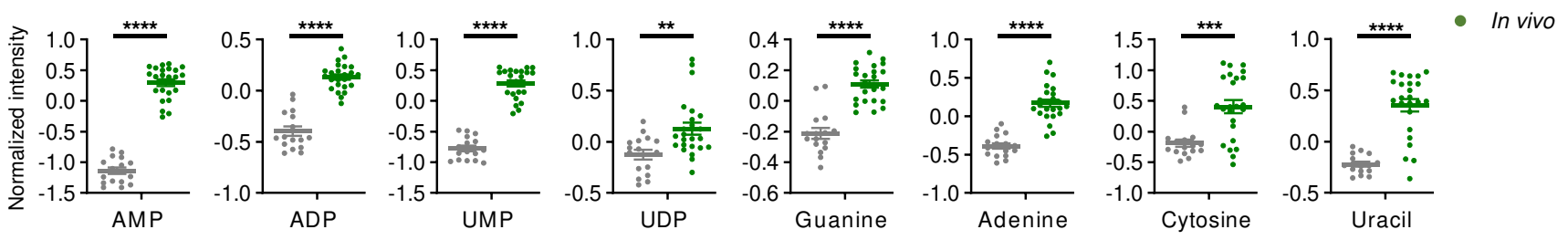


Figure 3

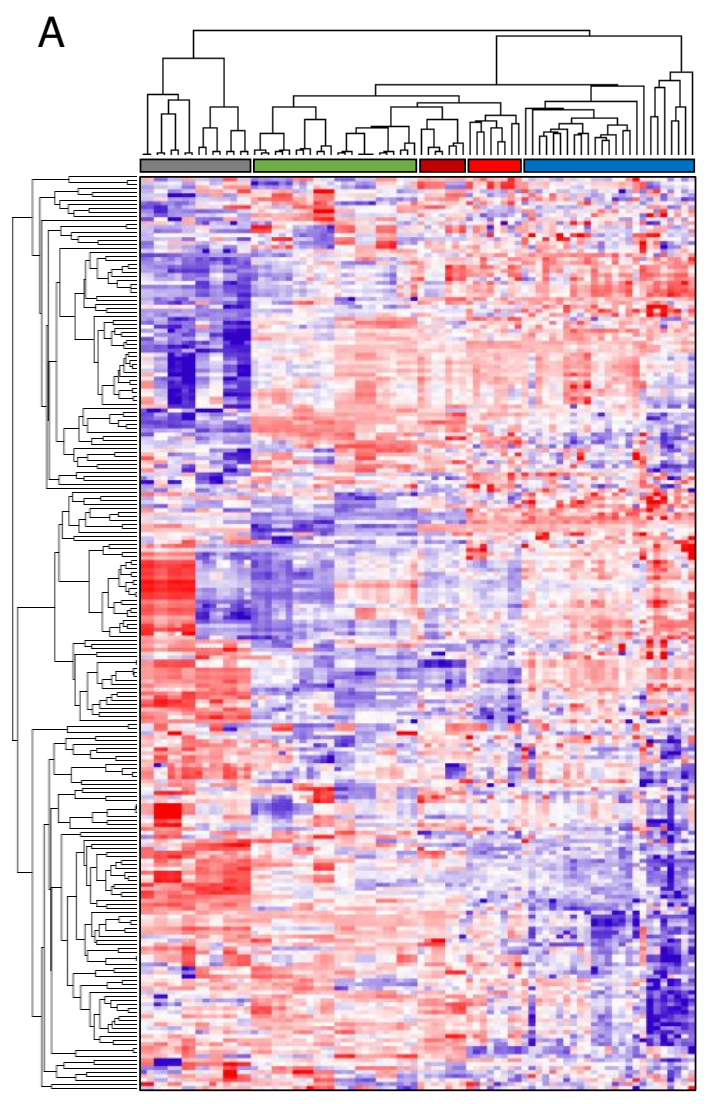

B

Sample Category

$\square$ Cell line in vitro

$\square$ Cell line in vivo

- PDXs

TNBC in Cluster 2

$\square$ Normal, ER+, and

TNBC in Cluster 1

Normalized

abundance

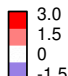

-1.5
-3.0
Metabolites abundance $\mathrm{ER}^{+}$vs TNBC

$(q<0.05$, SAM)

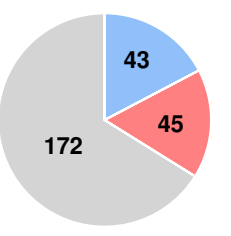

Total $=\mathbf{2 6 0}$

$\square$ High in $\mathrm{ER}^{+}$cells

$\square$ High in TNBC cells

$\square$ No changed

C
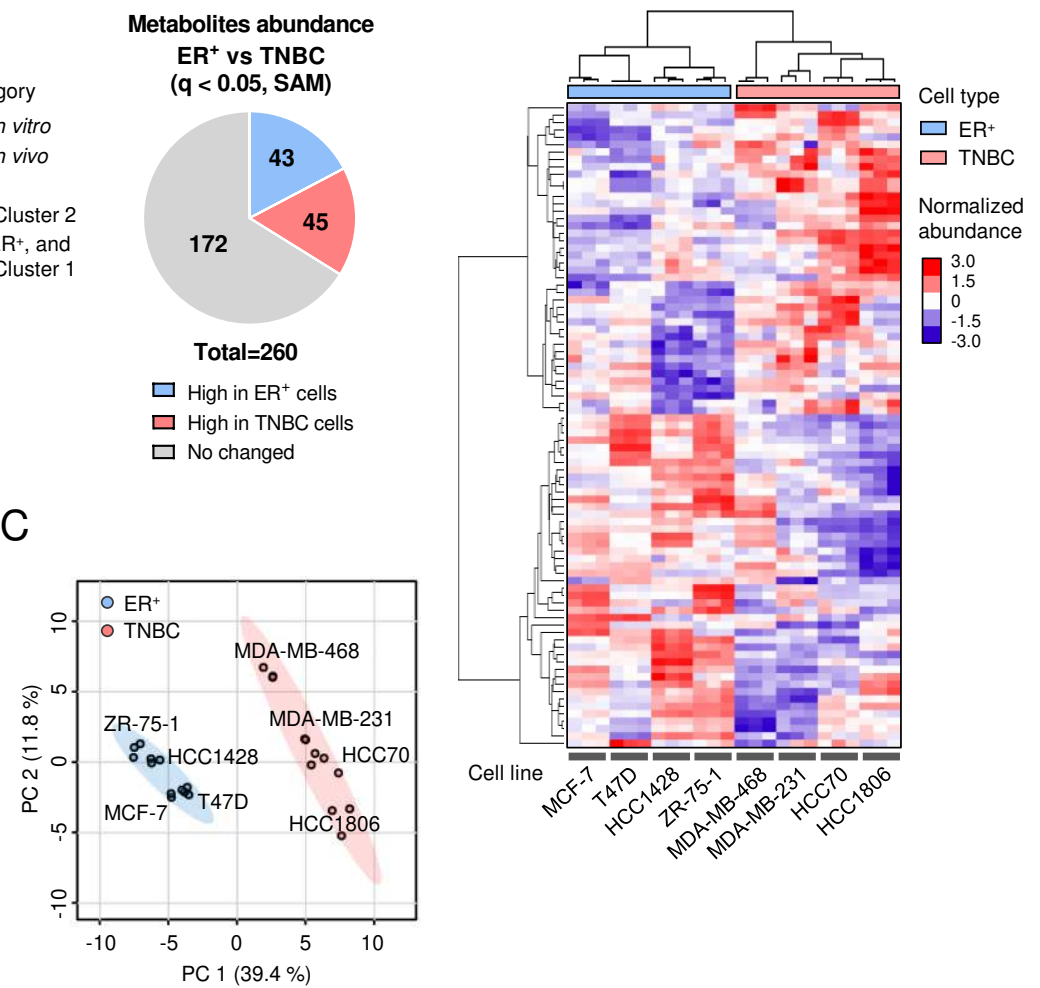

E

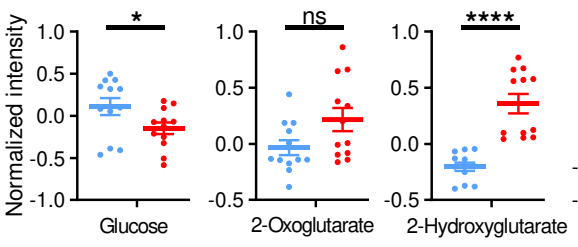

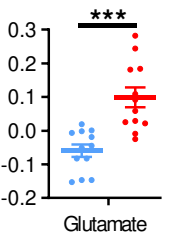

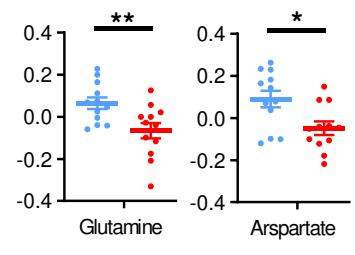

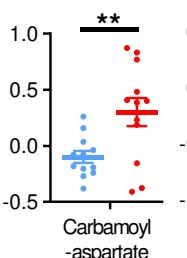
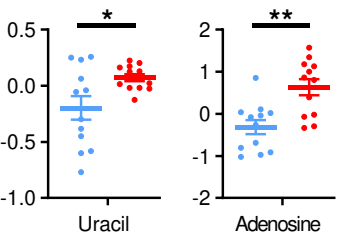

F
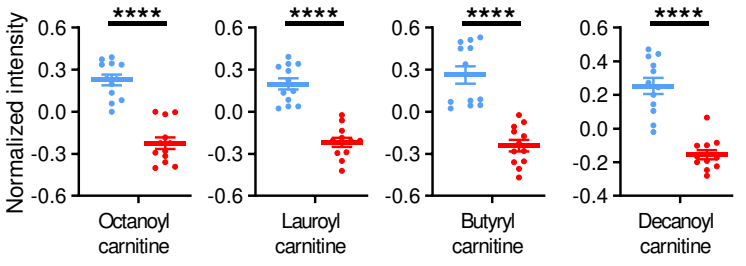

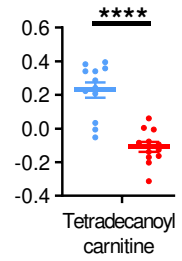

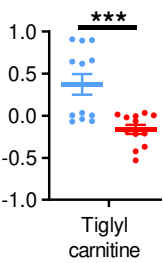

0.4
0.2
0.0
-0.2
-0.4
$3-$ hydroxy-isovaleryl
carnitine

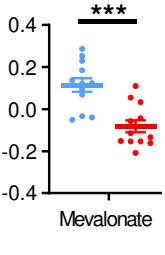

- $\mathrm{ER}^{+}$

- TNBC 


\section{Figure 4}

A

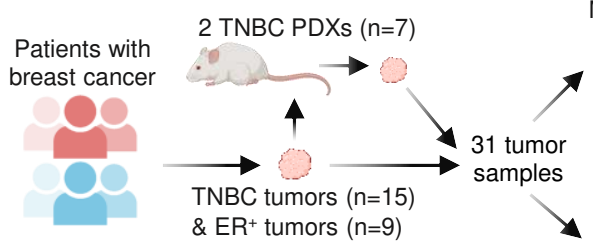

Metabolomics

\& $E R^{+}$tumors $(n=9)$

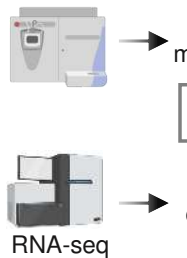

RNA-seq
B

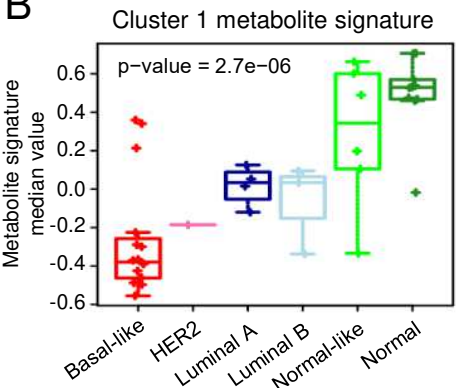

D
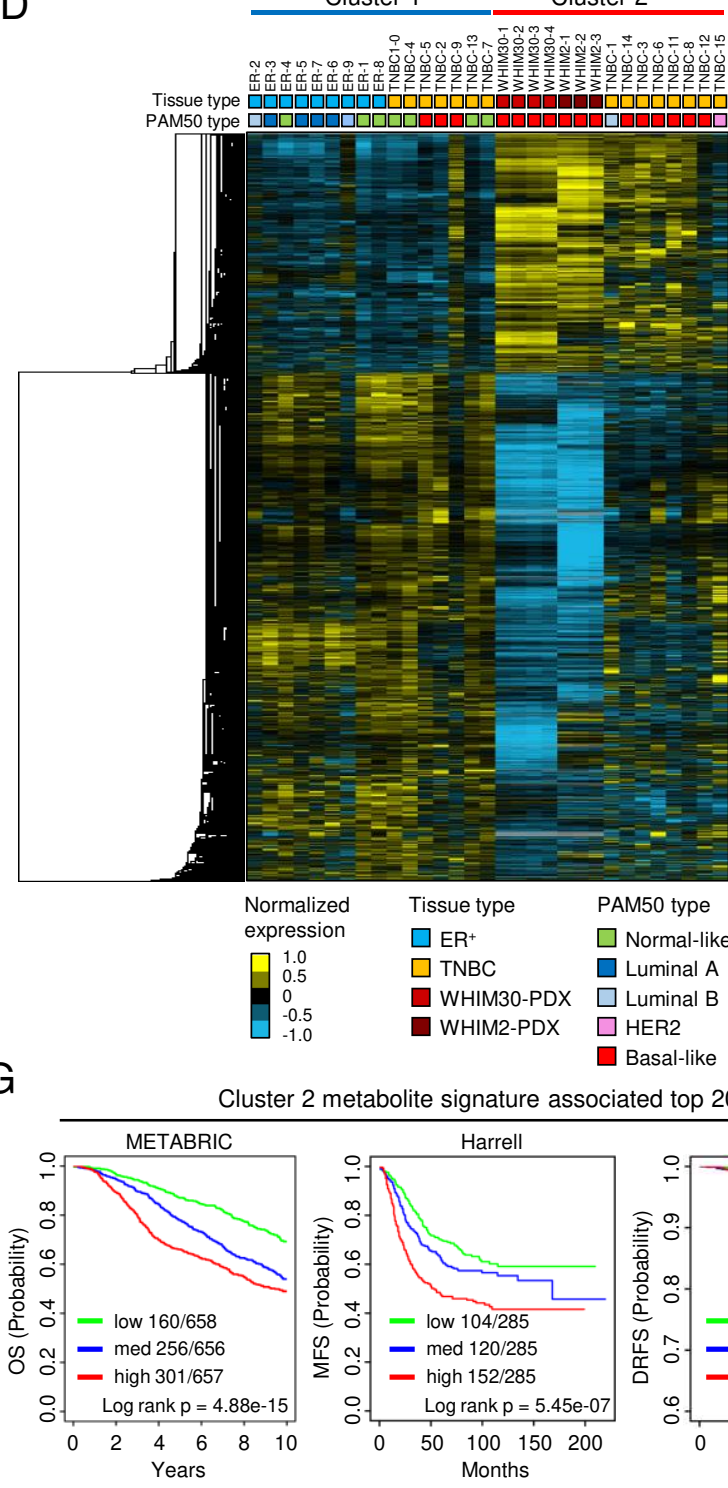

Cluster 2 metabolite signature

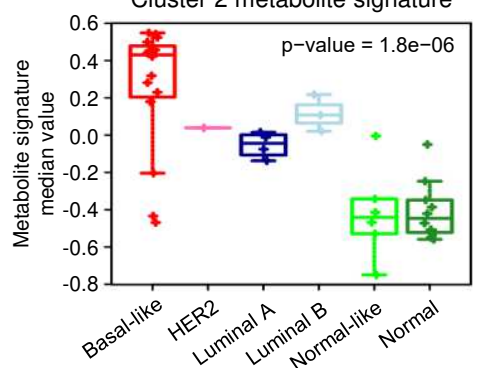

Cluster 2

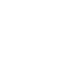

Unsupervised metabolic clustering Metabolite-gene
association $\downarrow$

Supervised gene Supervised gene
expression profiling

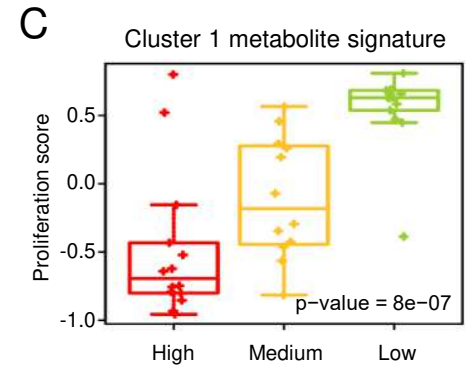

E

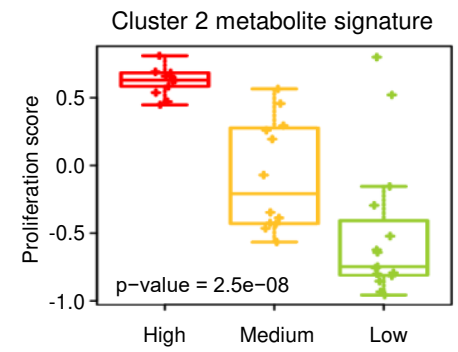

Cluster 2 metabolite signature associated top 200 genes_GO term
PAM50 subtype/ proliferation correlation

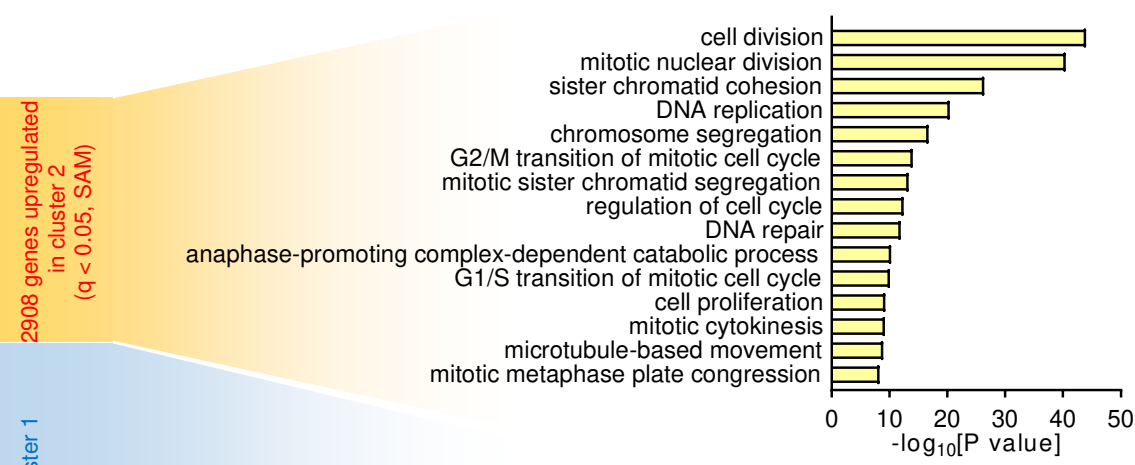

Cluster 1 metabolite signature

F associated top 200 genes GO term

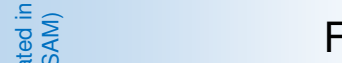

angiogenesis muscle contraction mone stimulus vasculogenesis regulation of cardiac con negative chemotaxis response to progesterone lipid transport leukocyte migration positive regulation of cell migration neural crest cell migration establishment of endothelial barrier cell surface receptor signaling pathway muscle contraction

negative regulation of angiogenesis

$\begin{array}{lllll}0 & 2 & 4 & 6 & 8\end{array}$

$\mathrm{H}$

Cluster 1 metabolite signature associated top 200 genes
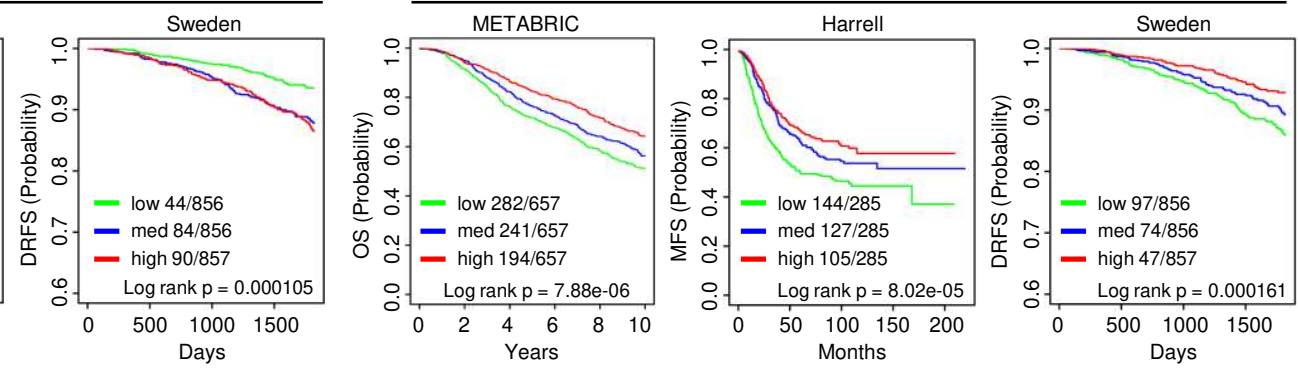
Figure 5

A

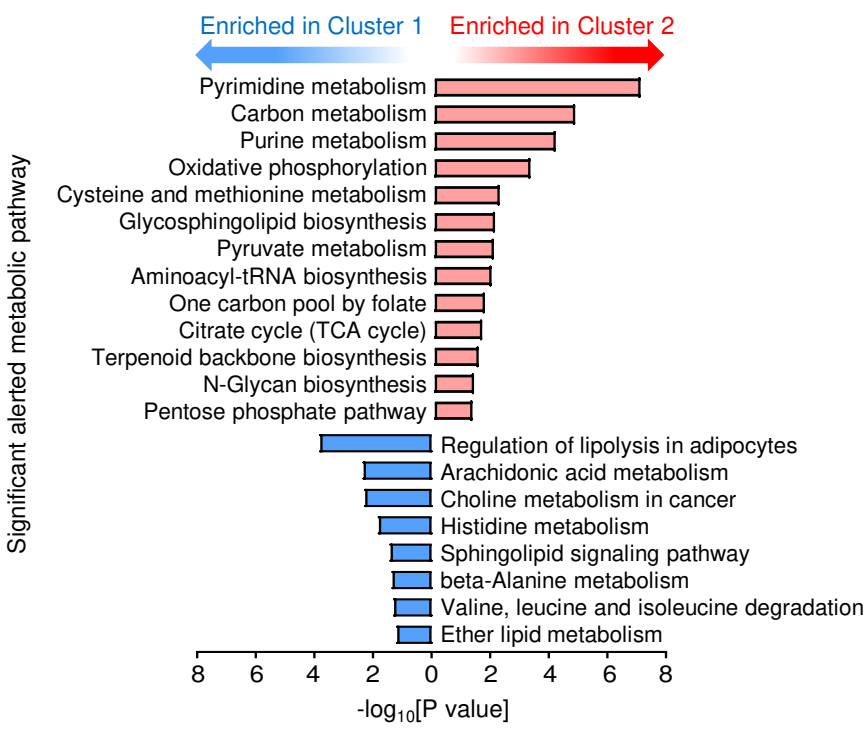

C

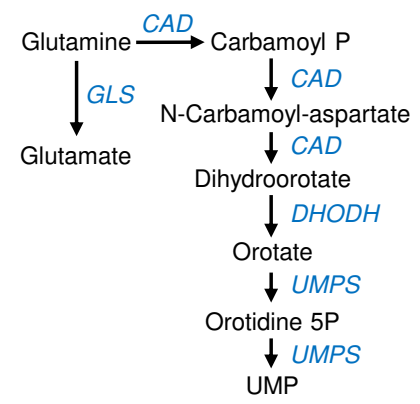

$\mathrm{D}$

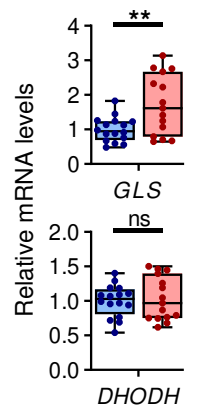

$\mathrm{F}$

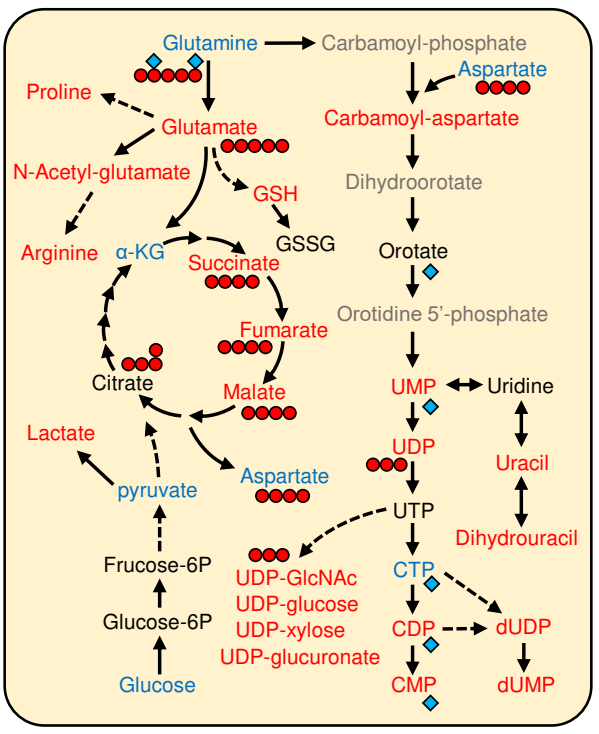

Metabolite annotation Labelling patterns

high in Cluster 1 No change $\quad 0{ }^{13} \mathrm{C}$

high in Cluster 2 Not detected $\quad{ }^{15} \mathrm{~N}$-amide
$\mathrm{G}$

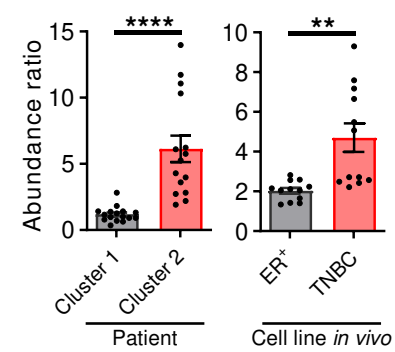

J Amino acids K

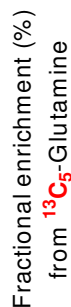

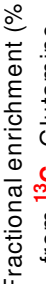

ㄴ.

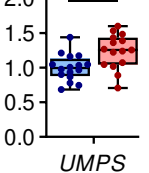

Glutamate/Glutamine

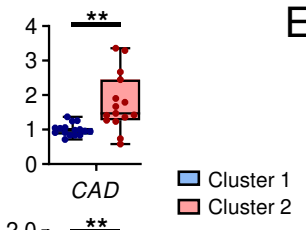

$E$

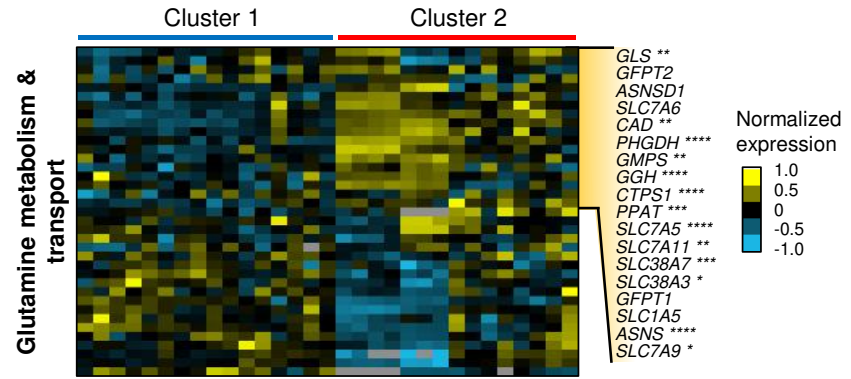

B

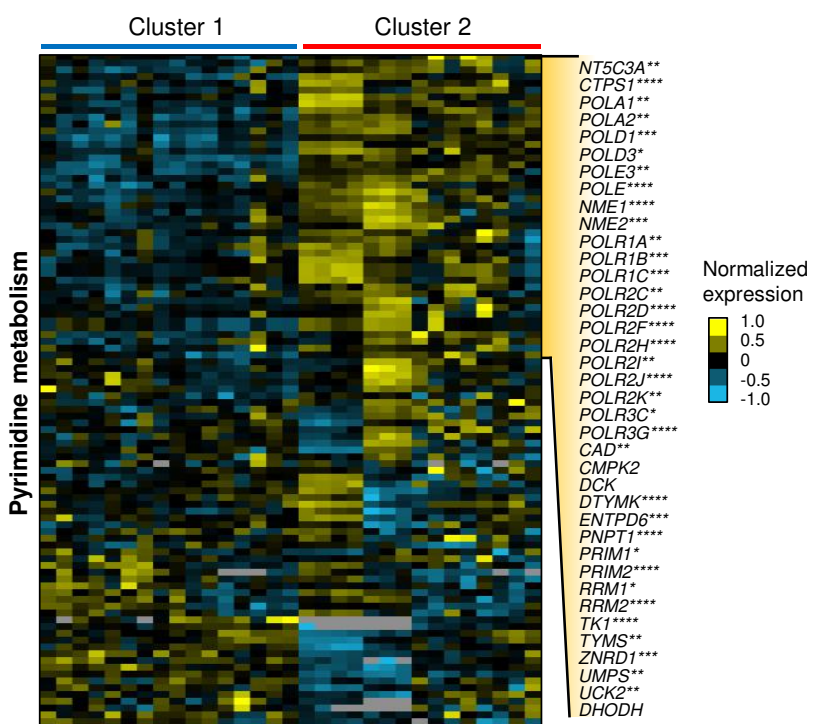

H Carbamoyl-aspartate/Glutamine I Carbamoyl-aspartate/Aspartate
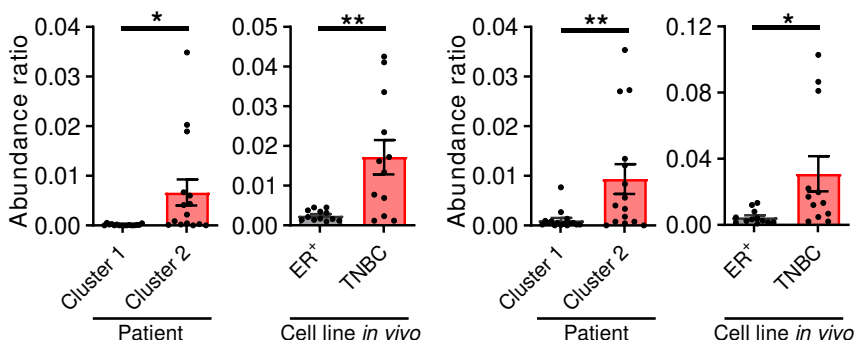
से

Cell line in vivo

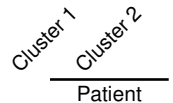
से

K TCA cycle\& Glutathione

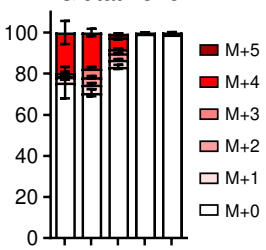
20
L Nucleotides\& Nucleotide sugar

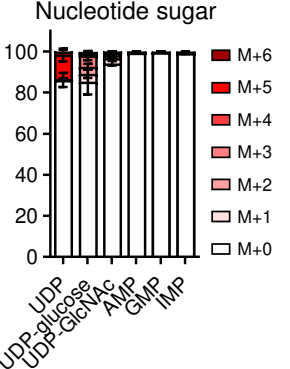

$\mathrm{M}$

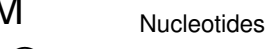

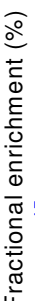

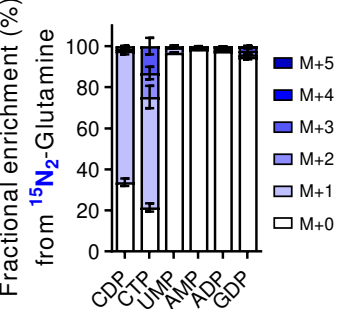


Figure 6

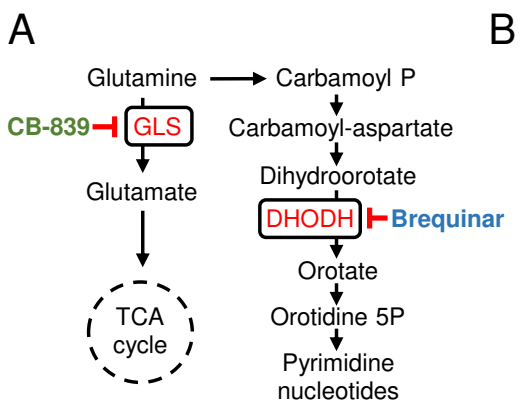

E
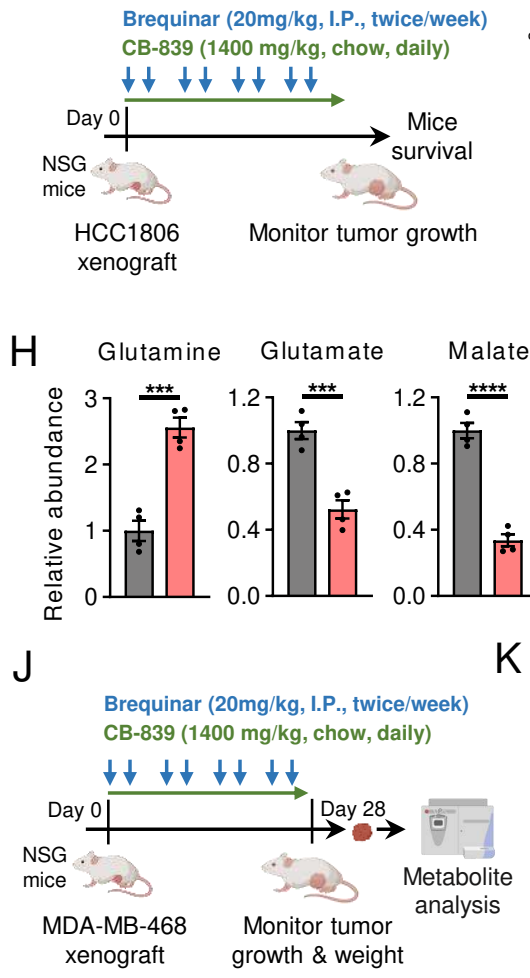

F
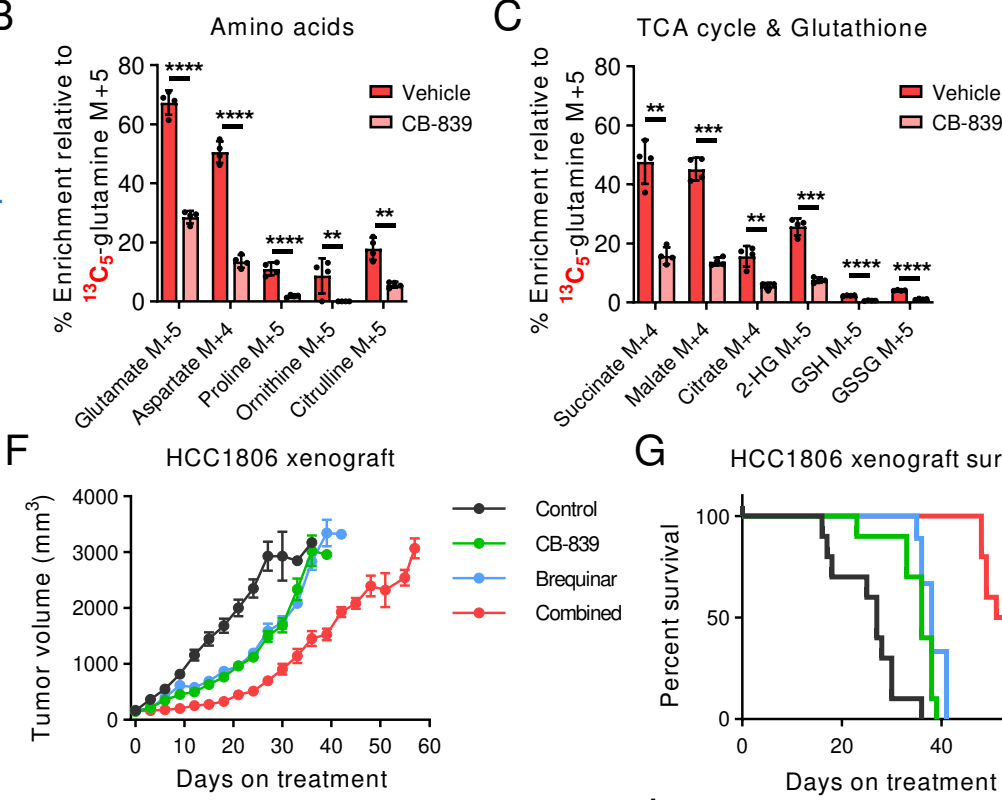

D

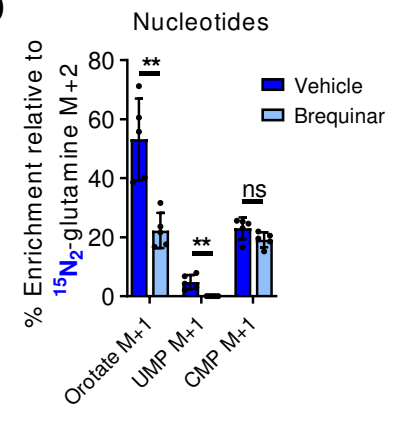

G HCC1806 xenograft survival
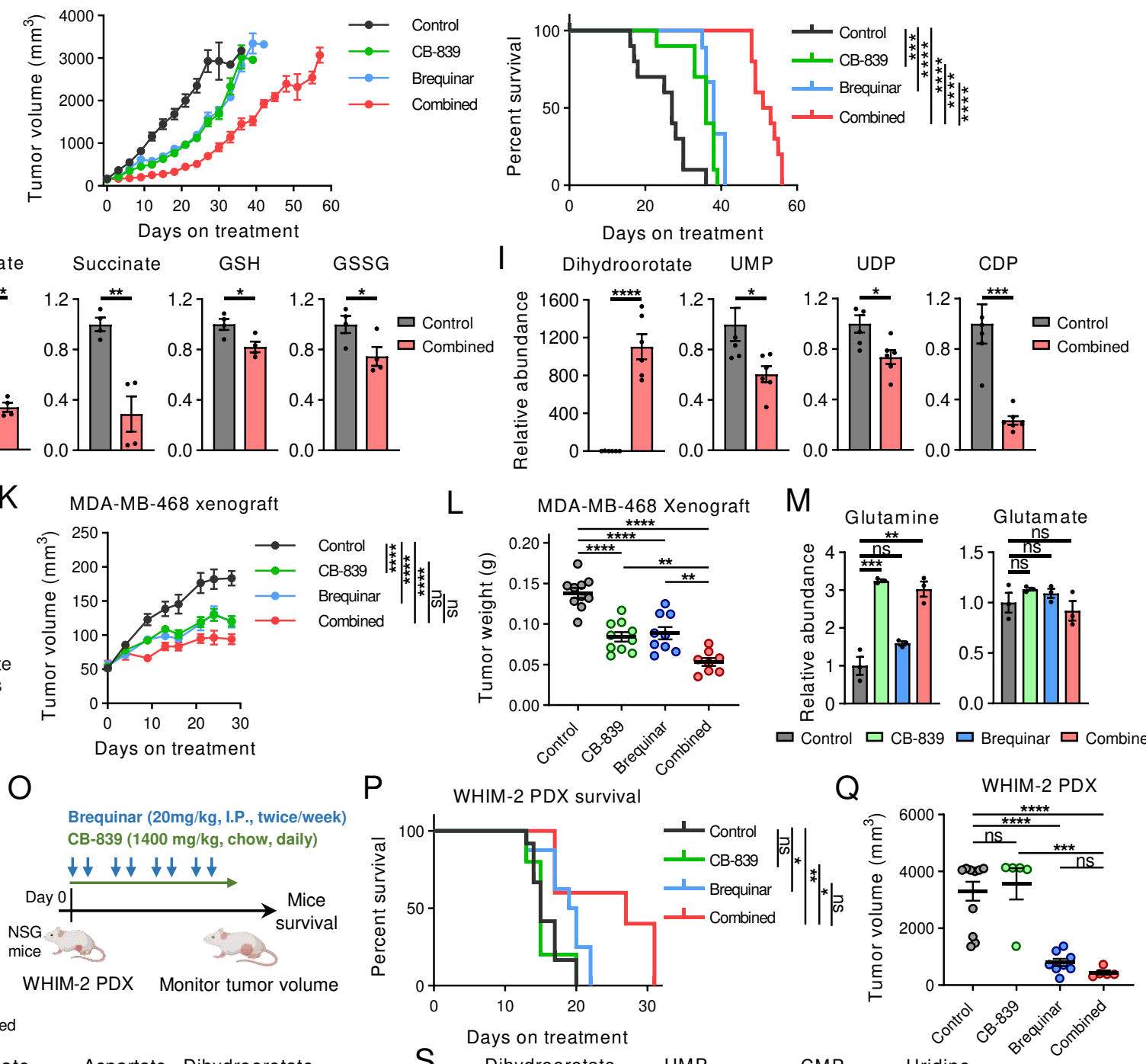

$\mathrm{N}$ Carbamoyl-aspartate Dihydroorotate
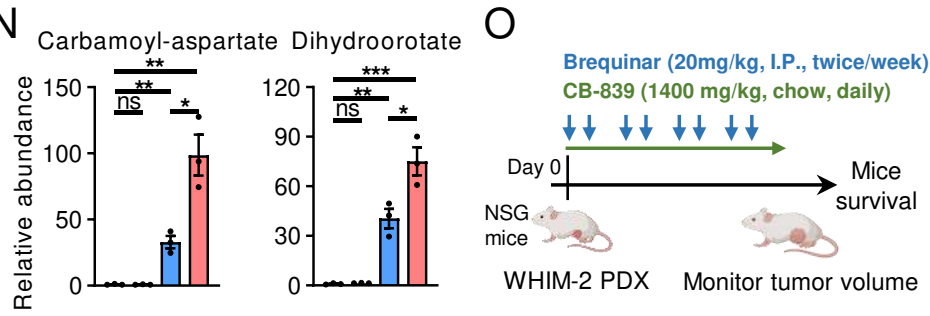

$\square$ Control $\square$ CB-839 $\square$ Brequinar $\square$ Combined

R Glutamine Glutamate Malate Aspartate Dihydroorotate

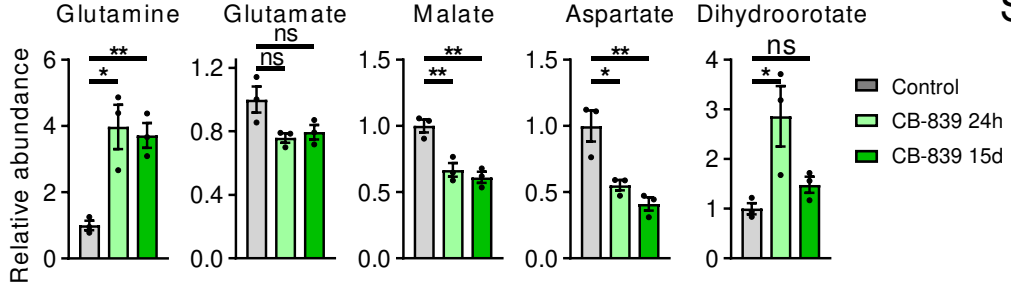

S Days on treatment
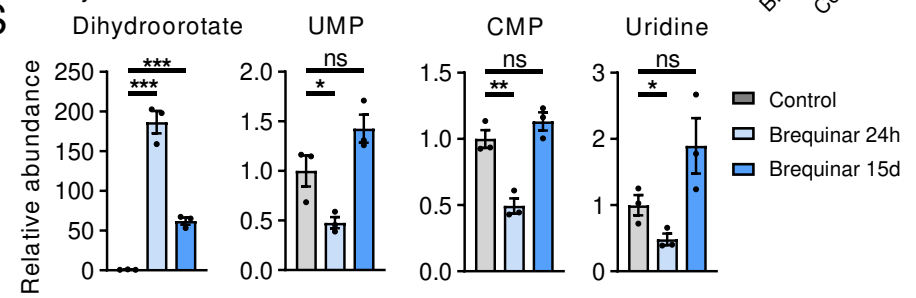
Figure 7

A

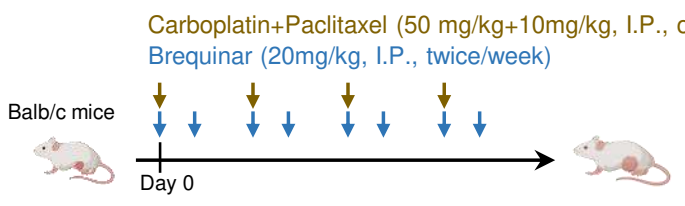

Five p53null TNBC murine tumor line xenografts

C

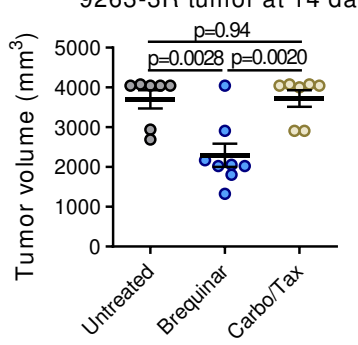

E T11 tumor at 14 day
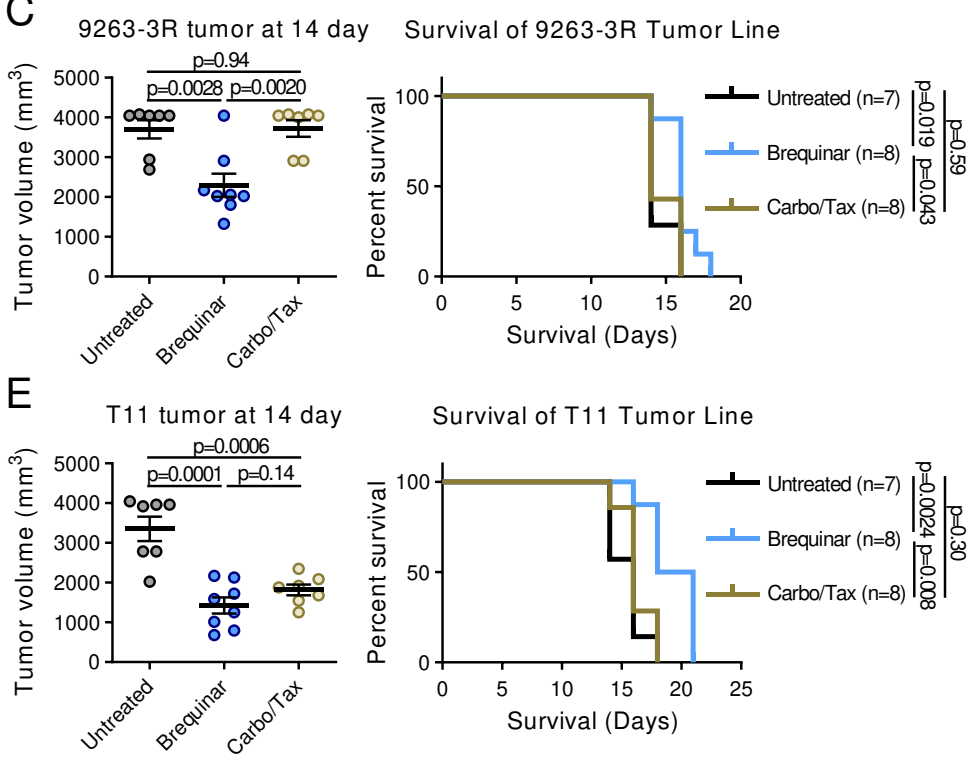

nitor tumor volume and survival

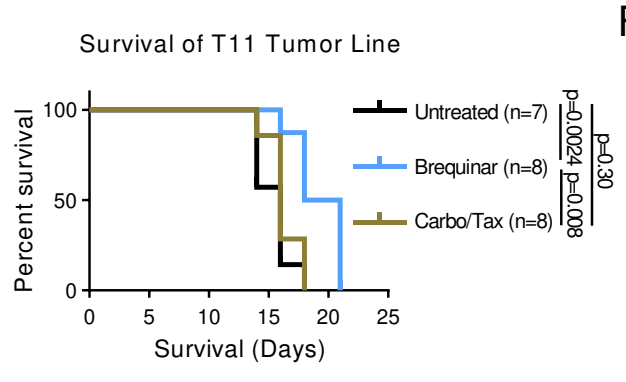

B

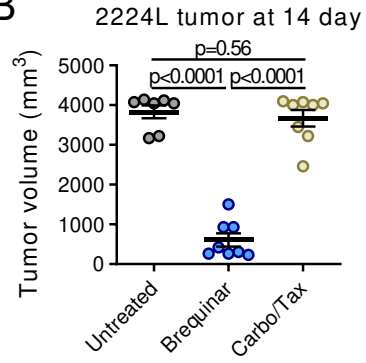

Survival of 2224L tumor line

D 2225L tumor at 14 day
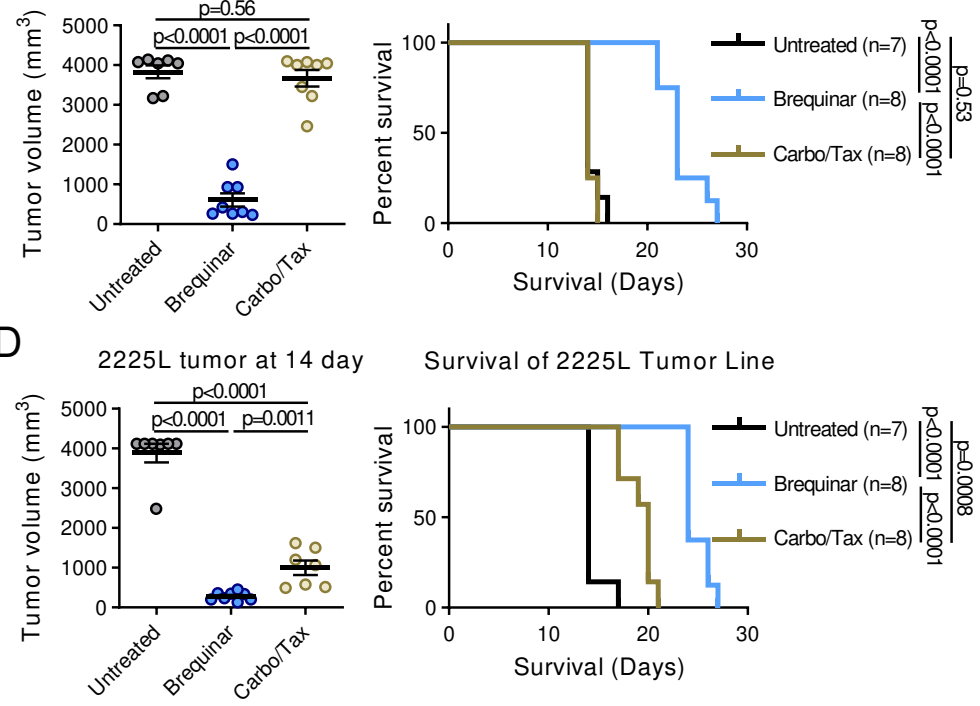

F

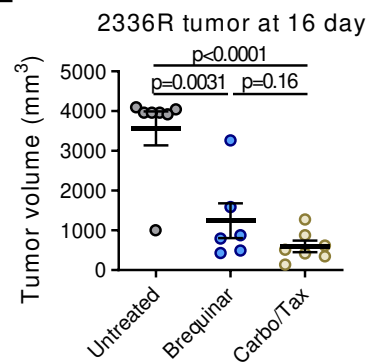

Survival of 2225L Tumor Line

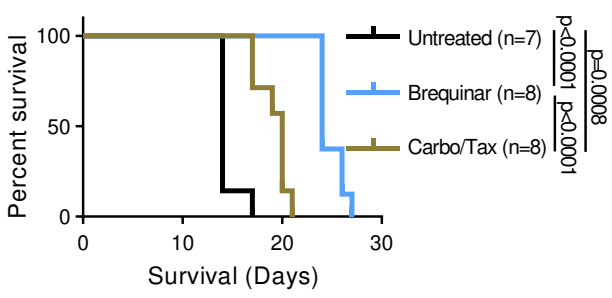

Survival of $2336 \mathrm{R}$ Tumor Line

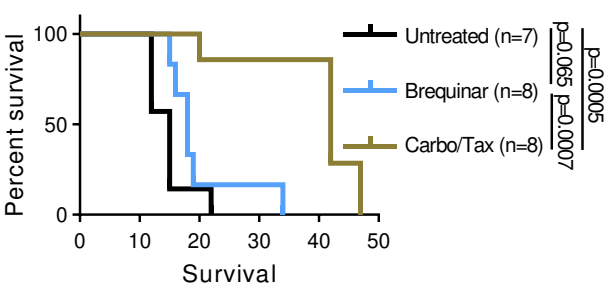


Figure S1

A

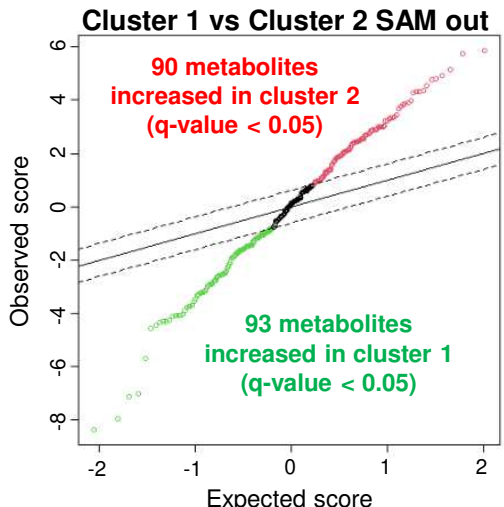

B
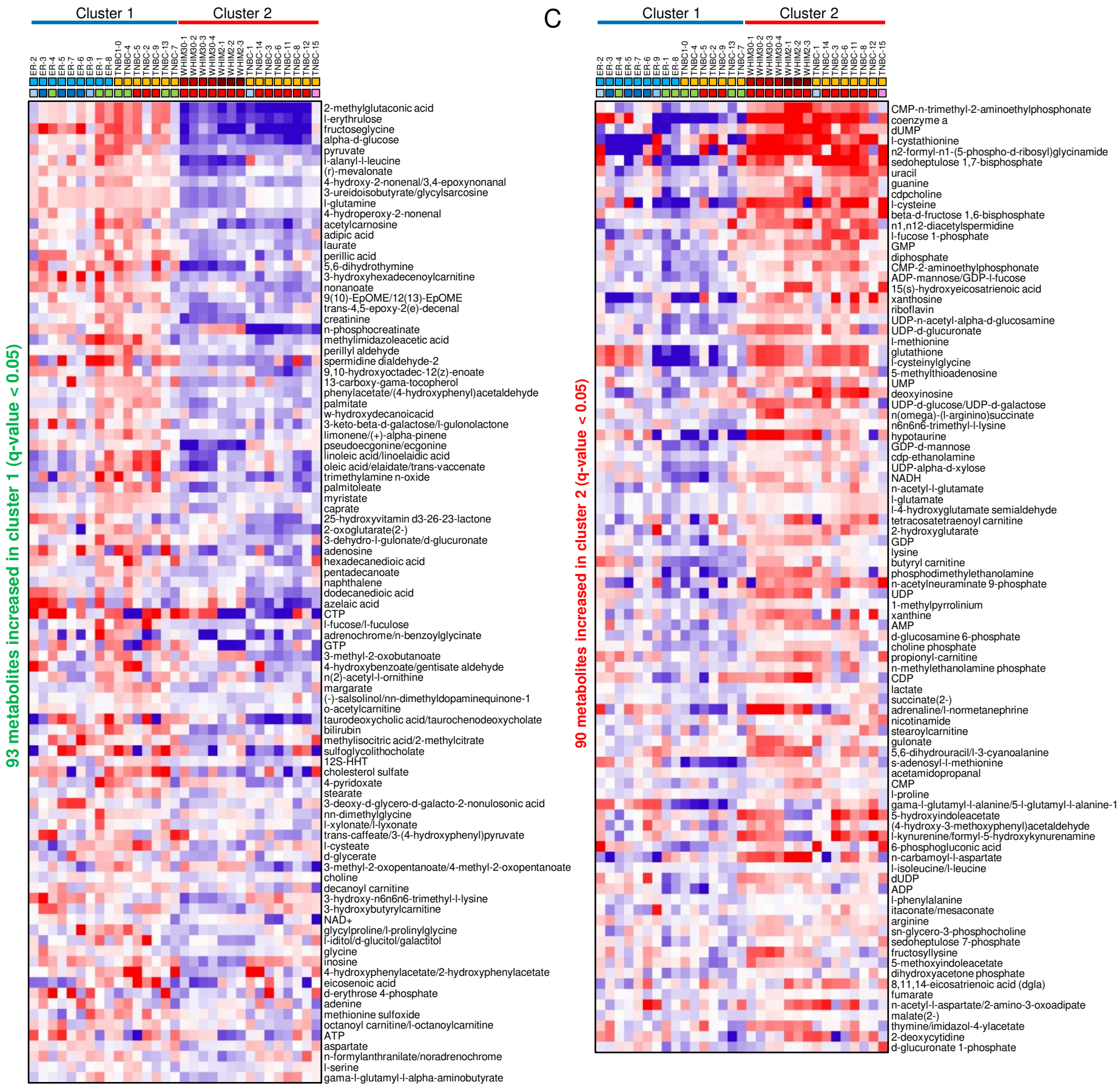

Figure S1. Top metabolites signatures enriched in breast cancer patients stratified by hierarchical clustering. (A) Significance Analysis for Microarrays (SAM) analysis revealed differential abundant metabolites (q-value $<0.05$ ) that altered in Cluster 1 or Cluster2 samples. (B and $\mathbf{C}$ ) Heatmap of top 93 metabolites enriched in cluster 1 (B) and top 90 metabolites enriched in cluster 2 (C). 
Figure S2

A

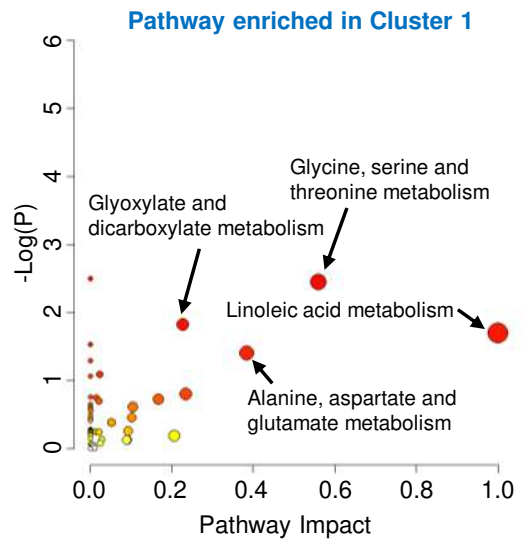

C

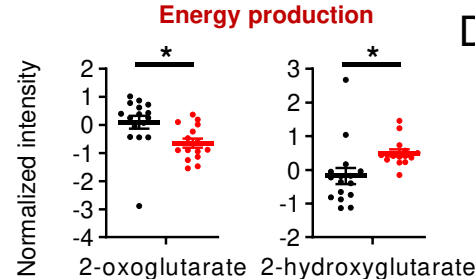

B

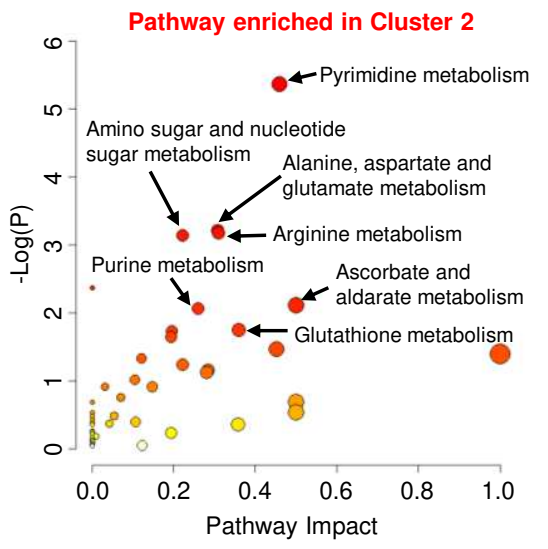

D

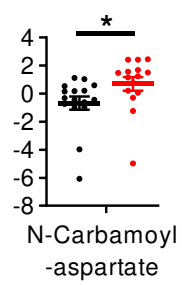

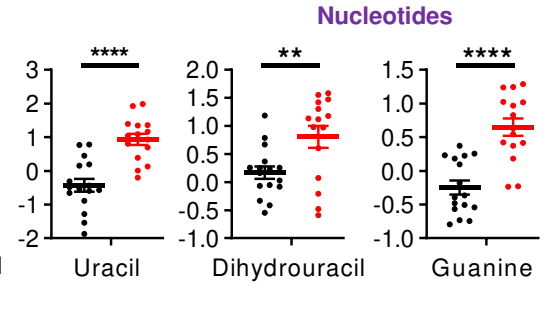

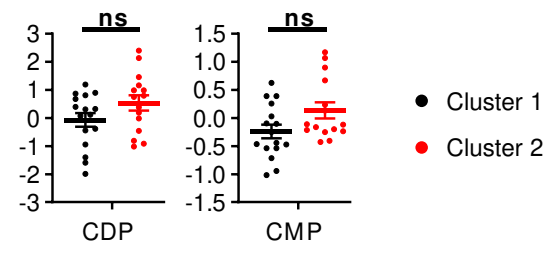

E

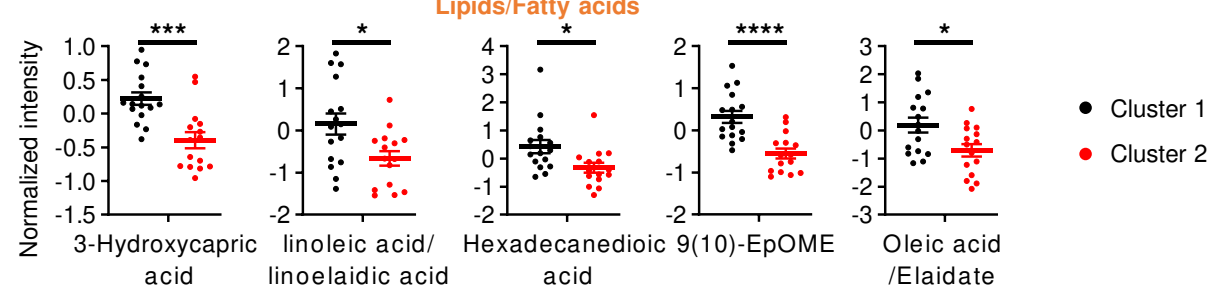

$\mathrm{F}$

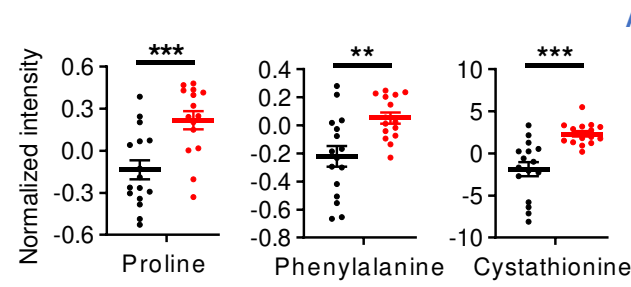

Amino acids
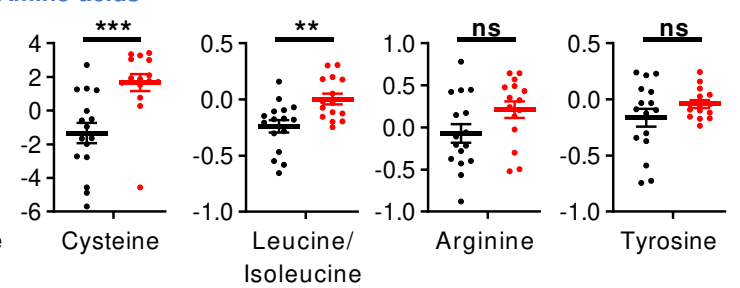

- Cluster 1
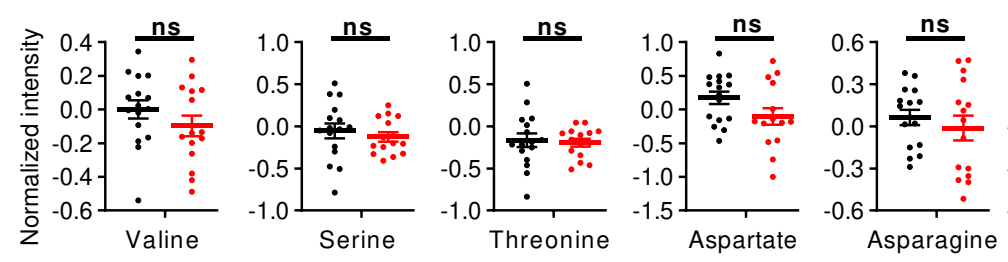

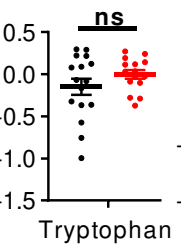

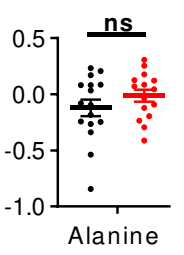

- Cluster 2

Figure S2. Metabolic pathways and representative metabolites between Cluster 1 and Cluster 2 samples. (A and B) Pathway enrichment and topology analysis by MetaboAnalyst 5.0 using the top 93 metabolites enriched in cluster 1 (A) or top 93 metabolites enriched in cluster 2 (B). Representative pathways with p-value $<0.05$ were annotated. The Kyoto Encyclopedia of Genes and Genome (KEGG) compound database was used as the reference metabolic pathway database. (C-F) Relative normalized intensity levels of representative metabolites involved in energy production (C), nucleotides (D), lipids/fatty acids (E), and amino acids (F) were showed. Data are represented as mean \pm standard error of the mean (SEM), two-tailed Student's t-test; ${ }^{*} p<0.05,{ }^{* *} p<0.01,{ }^{* * *} p<0.001,{ }^{* * *} p<0.0001$. 


\section{Figure S3}

A

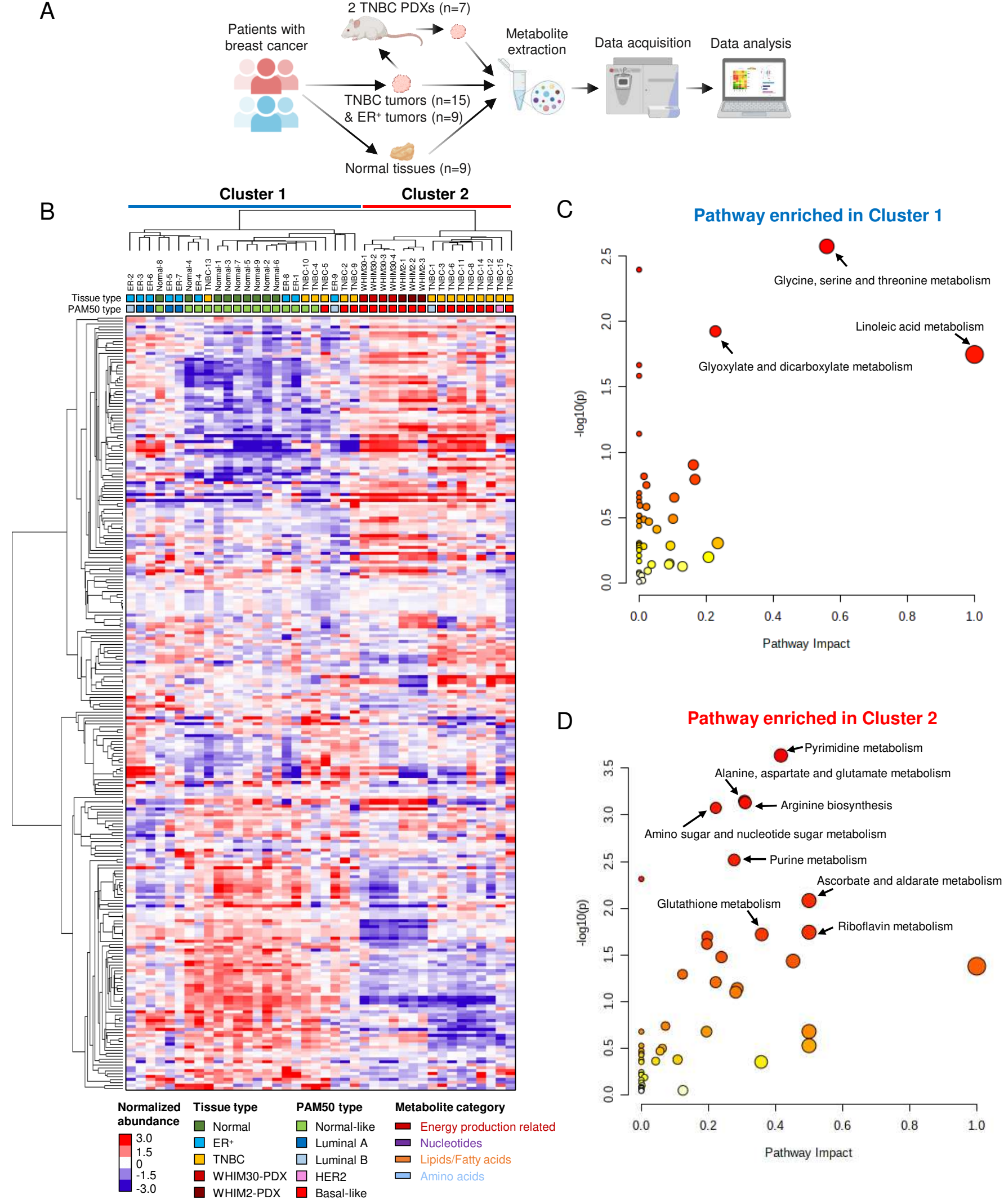

Figure S3. Metabolic profiling in tumors from breast cancer patient and PDXs. (A) Schematic of the experimental design for untargeted global metabolomics analysis in normal tissue, $\mathrm{ER}^{+}$, TNBC breast caner patient tumors and two TNBC patient-derived xenografts (PDXs) tumors. (B) Unsupervised hierarchical clustering heatmap examines global metabolic profiles in 40 normal tissue, ER ${ }^{+} / \mathrm{TNBC}^{2}$ patient tumor samples and PDX tumors by using the normalized metabolite intensity. Note the normal tissues grouped with the ER+ tumors in Cluster 1. (C and D) Pathway enrichment and topology analysis by MetaboAnalyst 5.0 using the top metabolites enriched in cluster 1 (C) cluster 2 (D). Representative pathways with $p$-value $<0.05$ were annotated. 
Figure S4

A

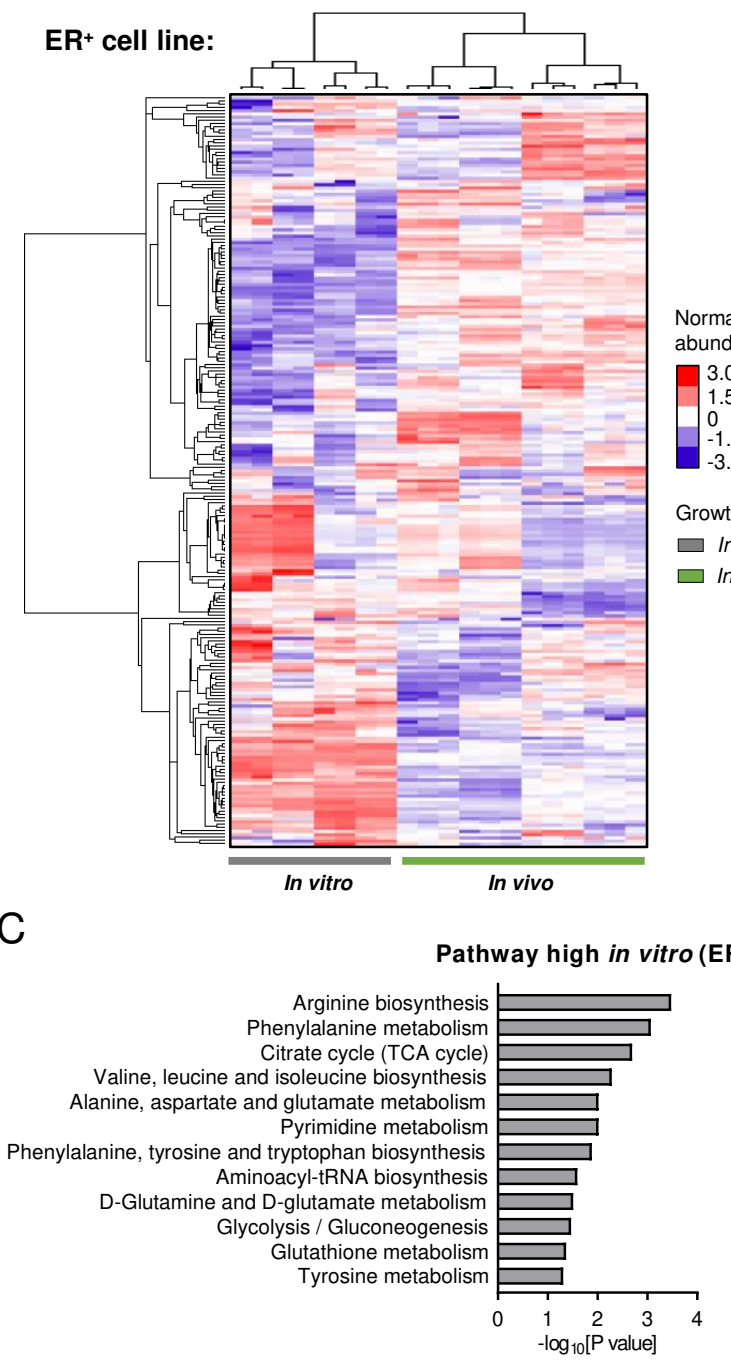

$\mathrm{E}$ Pathway high in vitro (TNBC)
B
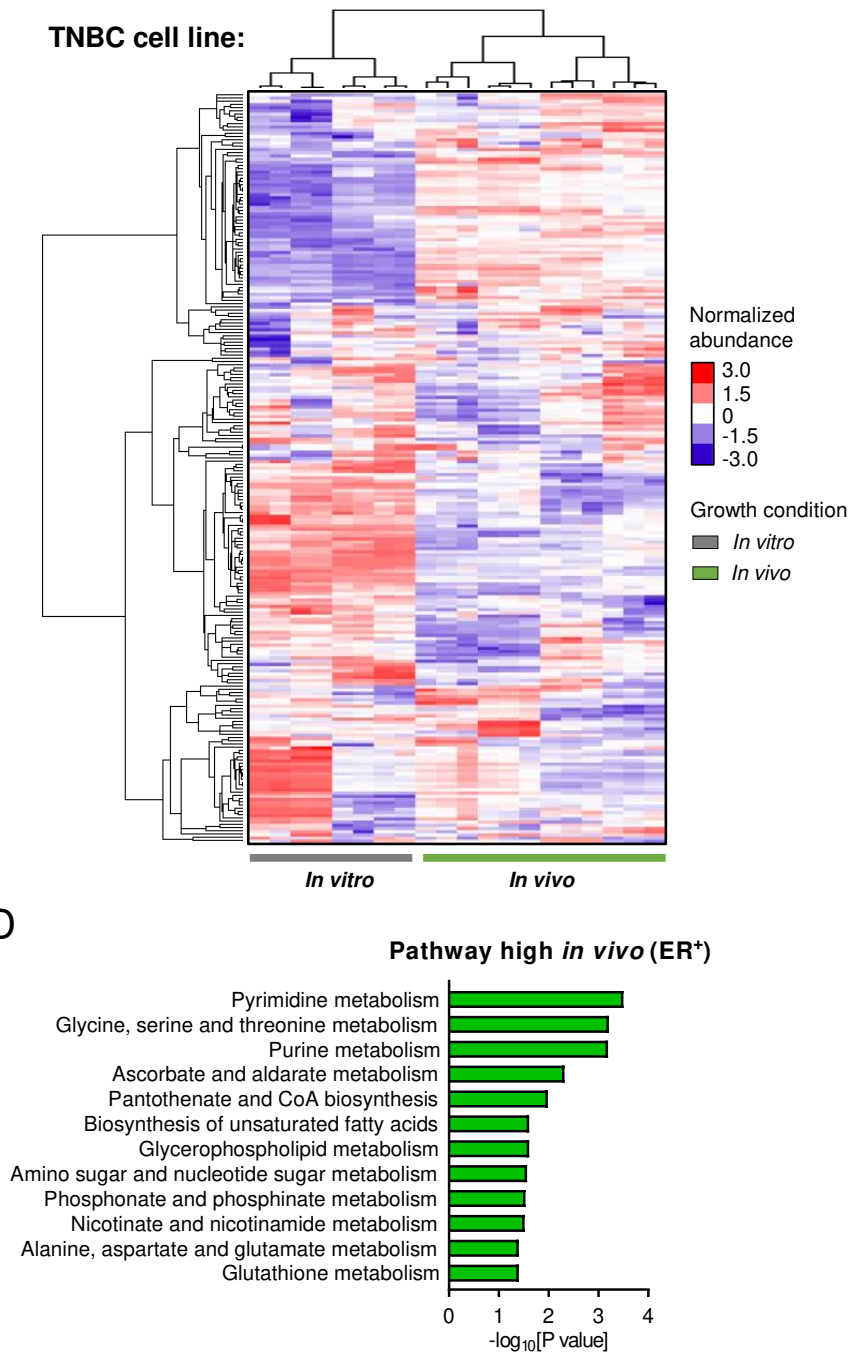

$\mathrm{F}$

Pathway high in vivo (TNBC)

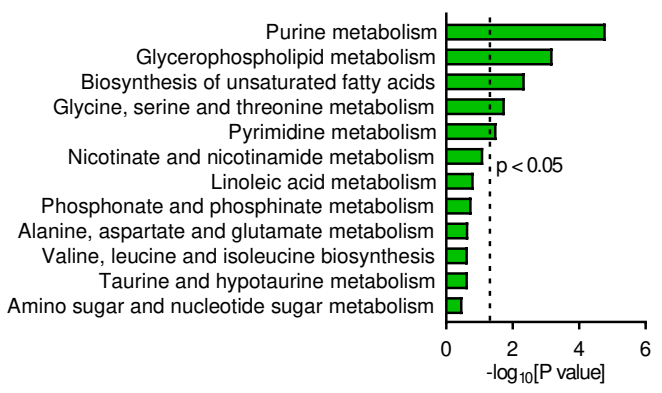

Figure S4. Metabolic profiling shows dramatic metabolic change in cell lines grown in vivo and in vitro. (A and B) Unsupervised hierarchical clustering heatmap of global metabolites in $\mathrm{ER}^{+}$ (A) or TNBC (B) cell lines grown in vivo and in vitro. (C and D) Histograms of metabolic pathway enrichment using statistical differed metabolites (SAM, q-value $<0.05$ ) that high in $\mathrm{ER}^{+}$cells grown in vitro (C) and in vivo (D). (E and F) Histograms of metabolic pathway enrichment using statistical differed metabolites (SAM, q-value $<0.05$ ) that high in TNBC cells grown in vitro $(\mathbf{E})$ and in vivo $(\mathbf{F})$. 
Figure S5

A

Alanine, aspartate and glutamate metabolism
Arginine biosynthesis
Cysteine and methionine metabolism
Amino sugar and nucleotide sugar metabolism
Purine metabolism
Pyrimidine metabolism
B
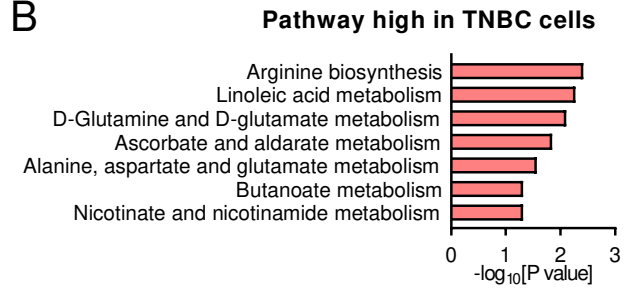

C

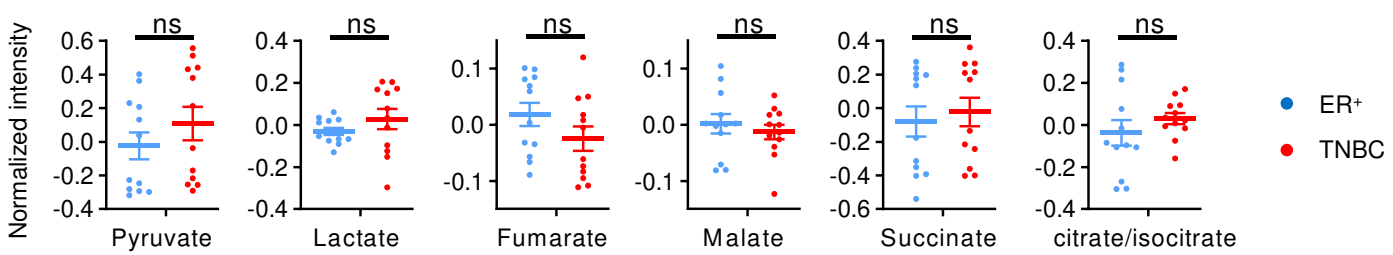

D

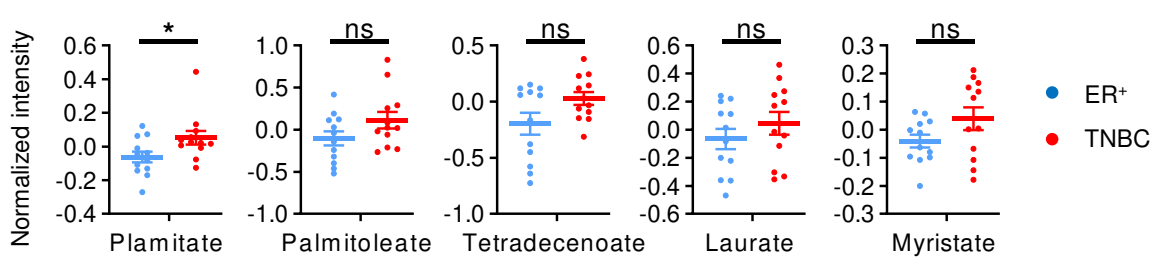

E

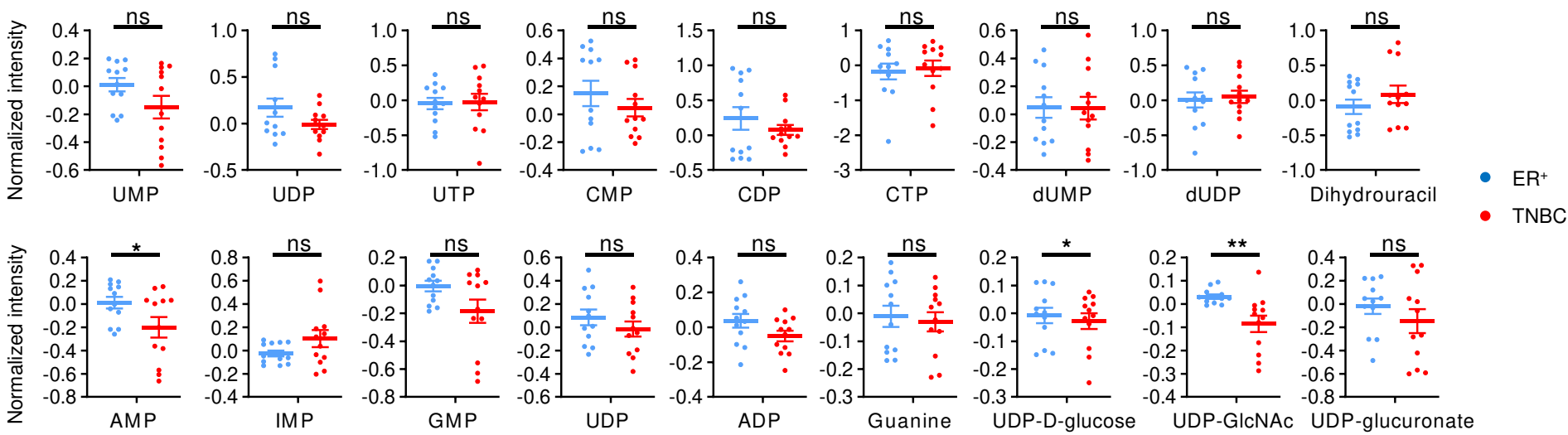

Figure S5. Metabolic profiling shows dramatic metabolic change in cell lines grown in vivo and in vitro. (A and $\mathbf{B}$ ) Histograms of metabolic pathway enrichment using statistical differed metabolites (SAM, q-value < 0.05) that high in $\mathrm{ER}^{+}(\mathbf{A})$ or TNBC (B) cell lines grown in vivo. (C-E) Relative normalized intensity levels of representative metabolites involved in energy production (C), lipids/fatty acids (D), and nucleotides (E) were showed. Data are represented as mean \pm standard error of the mean (SEM), two-tailed Student's t-test; ${ }^{*} p<0.05,{ }^{* *} p<0.01,{ }^{* * *} p<0.001,{ }^{* * * *} p<0.0001$. 
A

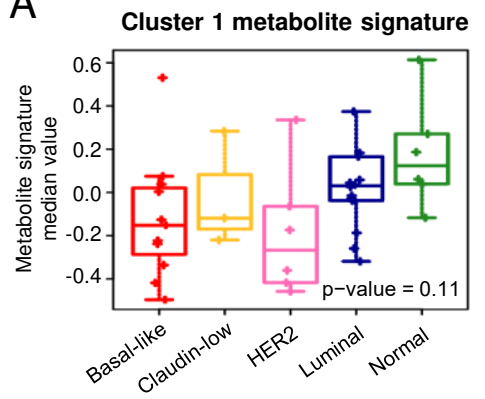

C

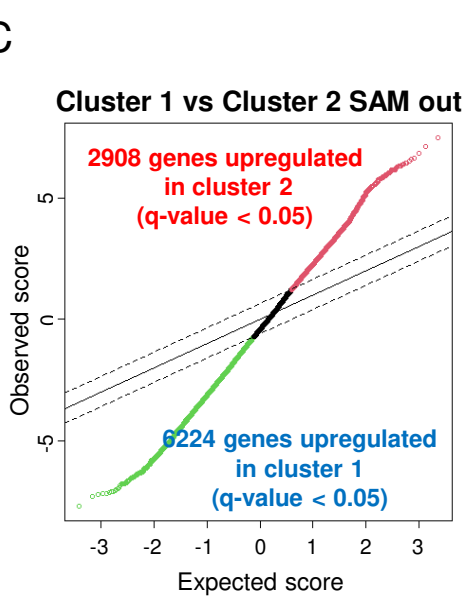

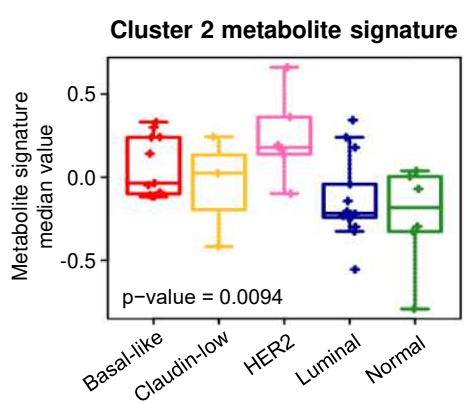

B
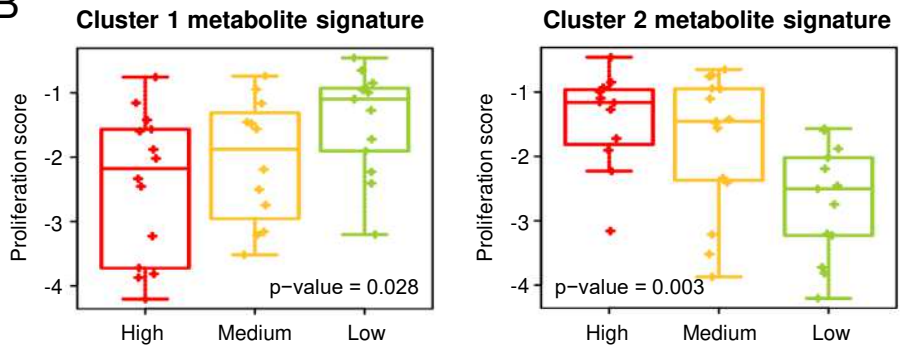

Validation Data Set (Brauer et al., 2013)

D

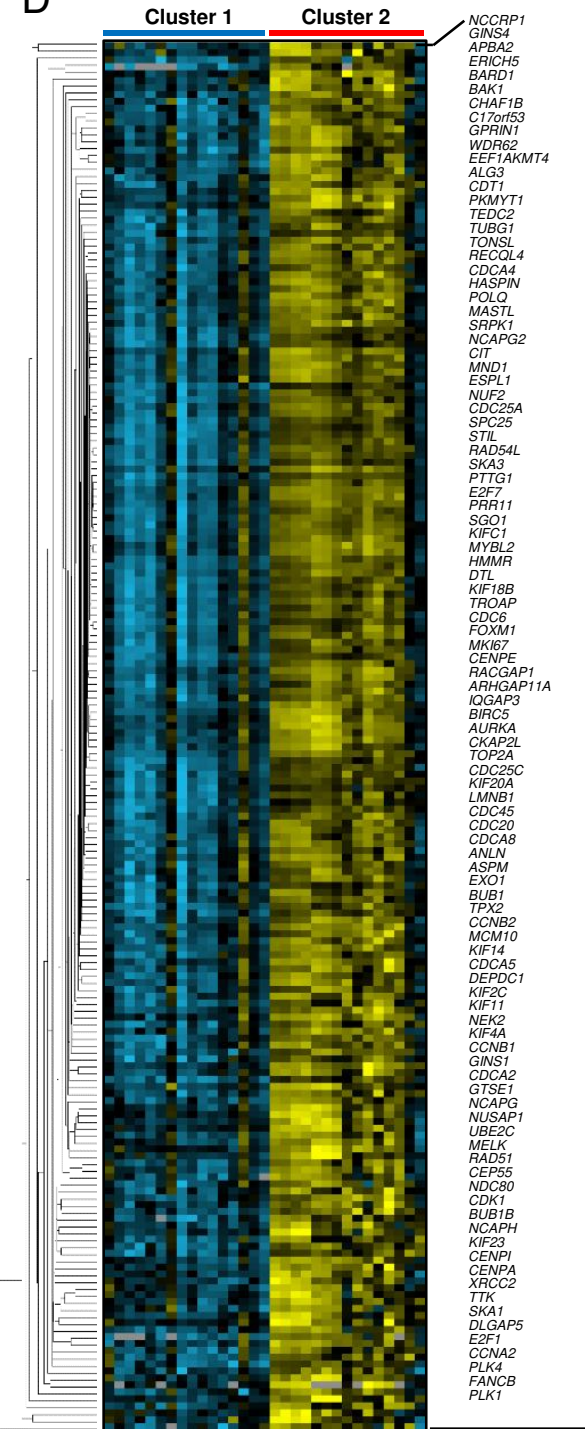

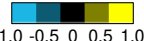

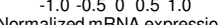

E

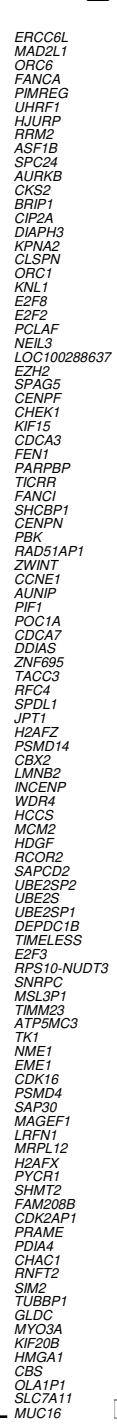

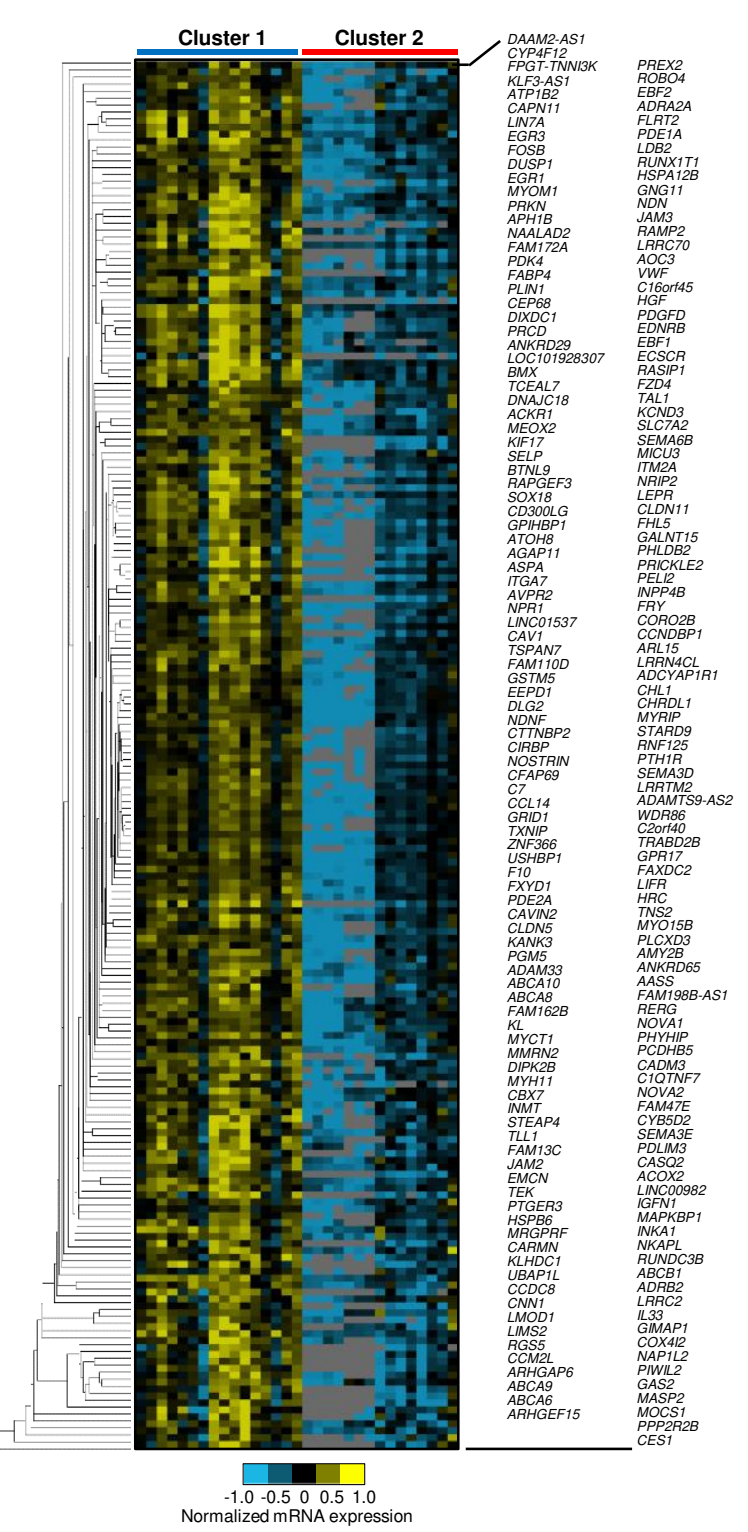

Figure S6. Integration analysis of transcriptional and metabolic signatures in patient tumors. (A) The PAM50 subtype correlation with the metabolite signatures from Cluster 1 and Cluster 2 patient samples in a validation data set of breast cancer patient samples from Brauer et al., 2013. (B) Correlation of proliferation score with the tertile median level of the two metabolite signatures from Cluster 1 and Cluster 2 patient samples in the validation data set of breast cancer patient samples. (C) Significance Analysis for Microarrays (SAM) analysis revealed differential expressed genes (q-value < 0.05) that altered in Cluster 1 or Cluster2 samples. (D and E) Heatmap of the top 200 genes that were highly expressed in Cluster 2 (D) or Cluster 1 (E) samples. 
Figure S7

A

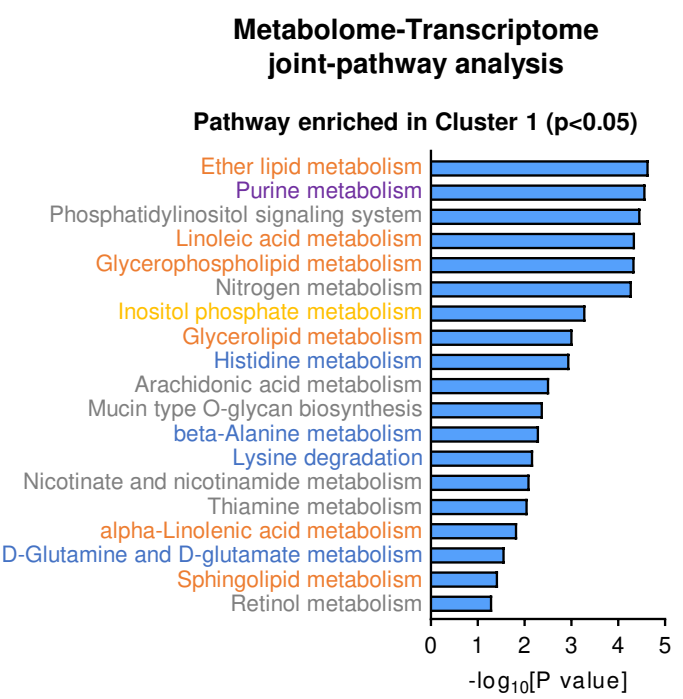

B

B Metabolome-Transcriptome joint-pathway analysis

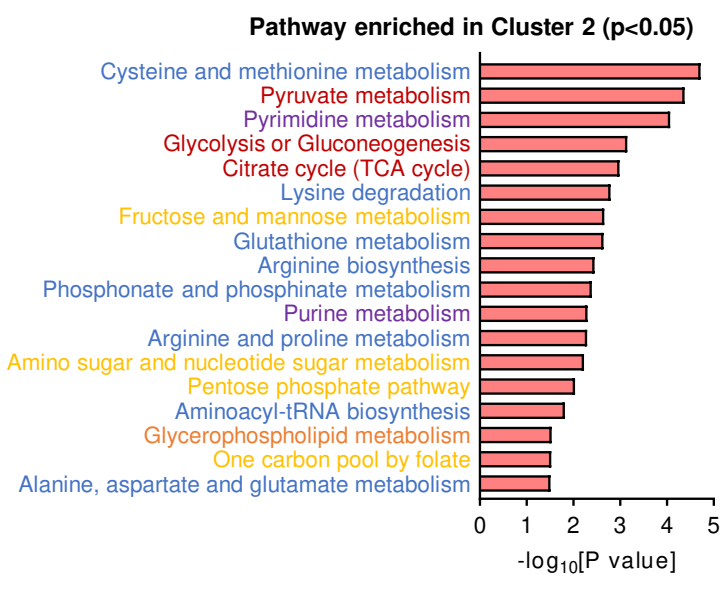

Pathway category

Figure S7. Integration analysis of transcriptional and metabolic signatures in patient tumors. (A and B) Metabolome-transcriptome joint-pathway analysis from MetabAnalyst 5.0 using the significantly accumulated metabolites and upregulated DE genes from the Cluster 1 (A) or Cluster 2 (B). The Kyoto Encyclopedia of Genes and Genome (KEGG) compound database was used as the reference metabolic pathway database. Significant enriched pathways ( $p$-value $<0.05)$ were showed. 
Figure S8

A

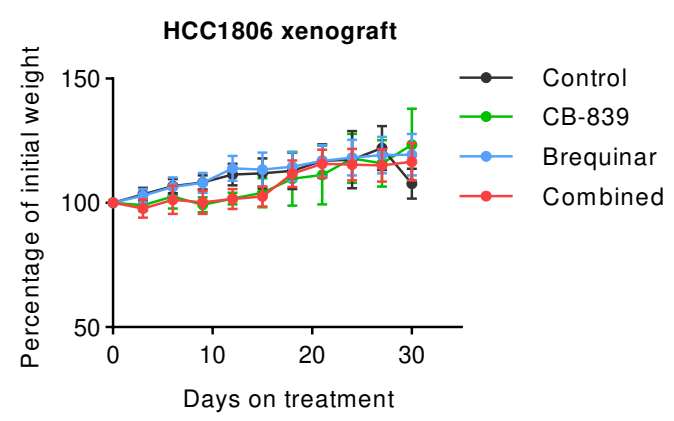

B

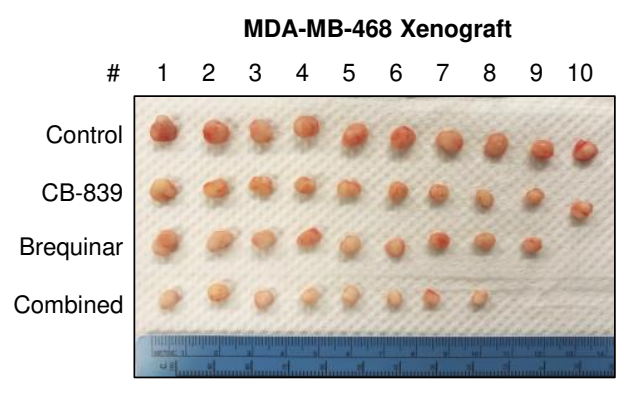

Figure S8. Targeting the pyrimidine and glutamate metabolism in TNBC cell line xenograft and PDX model. (A) Mouse body weight change during the treatment of the HCC1806 xenograft cohort. (B) Image of tumors after dissection at day 28 of the MDA-MB-468 xenograft cohort. 
Figure S9

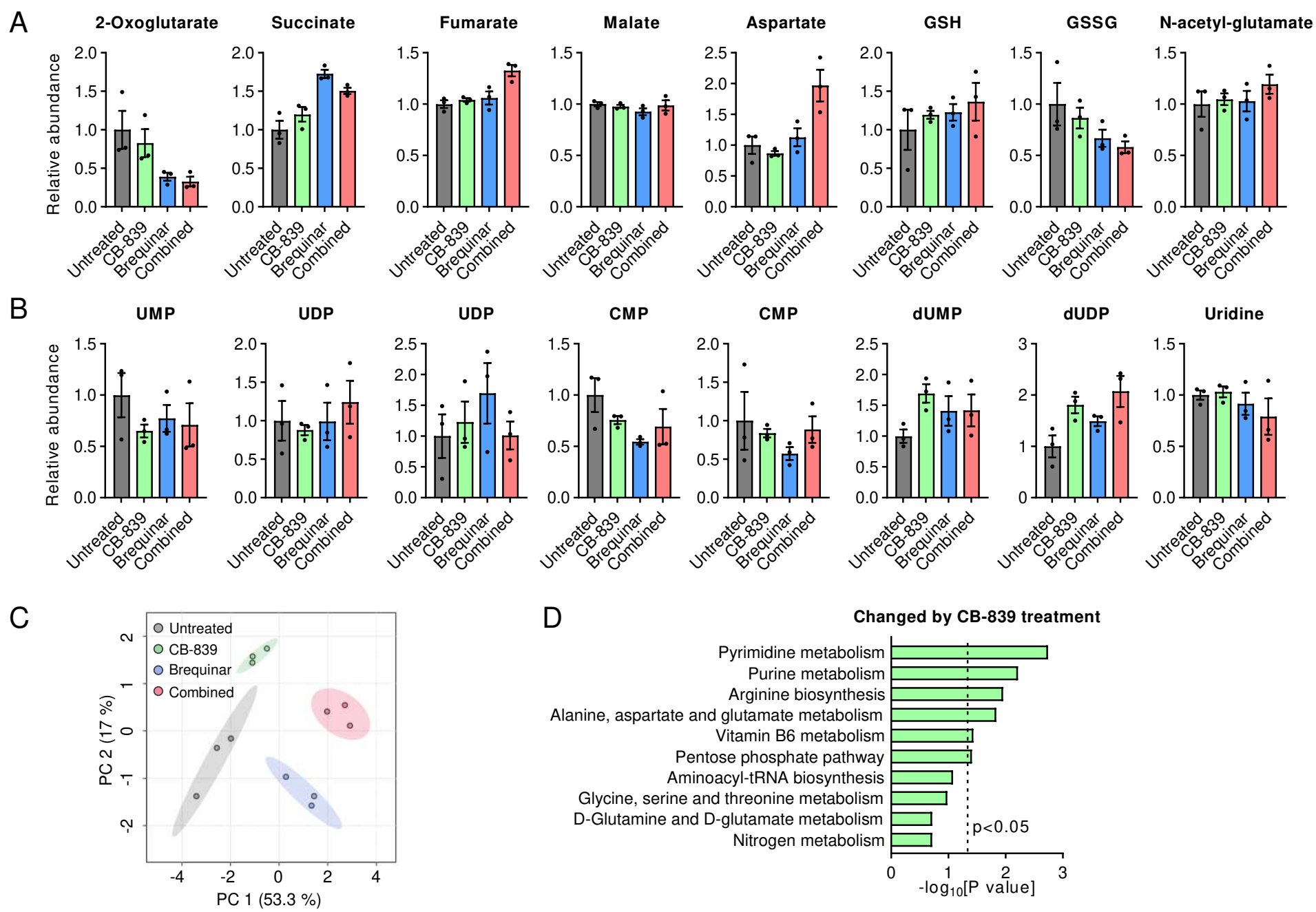

$E$

F

Changed by Brequinar treatment

Changed by Combination treatment

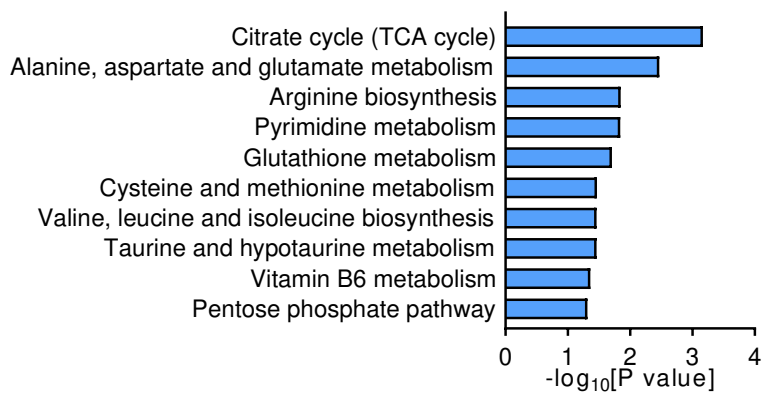

Figure S9. Metabolomics analysis of the drug treatment by targeting the pyrimidine and glutamate metabolism. (A and B) Relative intensity level of downstream metabolites involved in glutamine/glutamate metabolism (A) and pyrimidine metabolism (B) in the tumors of MDA-MB-468 xenograft treated with drug for 28 days. (C) Principal component analysis (PCA) using metabolomics data of MDA-MB-468 xenograft tumors with indicated treatment. (D-F) Histogram of metabolic pathway enrichment using statistical differed metabolites (SAM, q-value < 0.05) that changed in CB-839 (D), Brequinar (E), and combination (F) treated tumors versus control tumors in MDA-MB-468 xenograft. 